\title{
Mathematical Modeling of Tumor-induced Angiogenesis
}

\author{
Nikos Mantzaris, Steve Webb, \& Hans G. Othmer* \\ School of Mathematics ${ }^{\dagger}$ \\ University of Minnesota \\ Minneapolis, MN 55455
}

\section{Contents}

1 Introduction 3

2 Normal Vascular Physiology 5

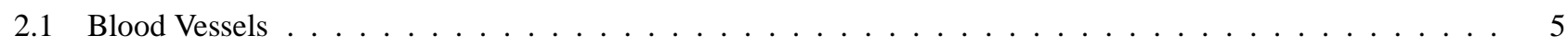

2.2 Extracellular Matrix . . . . . . . . . . . . . . . . . . . . . . . . . 5

3 Early Events in Angiogenesis 6

3.1 Signaling From Tumor To Vessel at the Onset of Angiogenesis . . . . . . . . . . . . . . . . 6

3.1 Transforming Growth Factor Beta $(\mathrm{TGF}-\beta) \ldots \ldots \ldots$

3.1 .2 Basic Fibroblast Growth Factor $(\mathrm{bFGF}) \ldots \ldots \ldots$

3.1.3 Vascular Endothelial Growth Factor (VEGF) . . . . . . . . . . . . . . . . . . . 7

3.2 Modifications of the ECM that Facilitate EC Migration . . . . . . . . . . . . . . . . . . 8

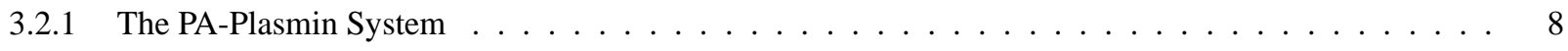

3.2.2 The MMP Family of Proteinases . . . . . . . . . . . . . . . . . . . . 9

4 Construction of a Growing Vessel 10

4.1 Cell Orientation to Extracellular Signals and Movement Through the ECM . . . . . . . . . . . . . . . 10

4.2 Capillary Lumen Formation . . . . . . . . . . . . . . . . . . . . . . 11

4.2 .1 Intracellular Lumen . . . . . . . . . . . . . . . . . . . . . . . 11

4.2 .2 Intercellular Lumen . . . . . . . . . . . . . . . . . . . . . . . 11

5 Maturation of the Neovasculature 12

5.1 Angiopoietins and Tie Receptors . . . . . . . . . . . . . . . . . . . . . . 12

5.2 Pericytes and Platelet-Derived Growth Factor . . . . . . . . . . . . . . . . . . . . . . . . 14

6 The Role of Macrophages in Angiogenesis 15

* All authors contributed equally to this work. Research supported in part by NIH Grant GM29123 and NSF Grants DMS0096312 and DMS0074043.

†Present addresses: NM: Department of Chemical Engineering, Rice University, Houston, TX; SW: Department of Mathematical Sciences, Loughborough University Leicestershire UK 
7 Angiogenesis Inhibitors 17

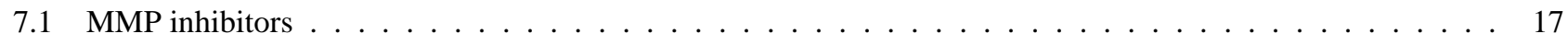

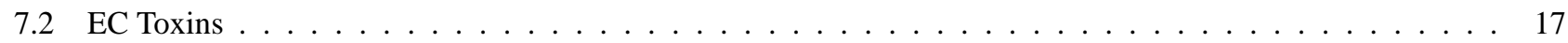

7.3 Angiogenesis Activator Blockers . . . . . . . . . . . . . . . . . . . . . 18

7.4 Integrin Binding Blockers . . . . . . . . . . . . . . . . . . . . . 18

7.5 Inhibitors as Fragments of Large Precursor Molecules . . . . . . . . . . . . . . . . . . . . . . . . . . 18

8 Mathematical Modeling 19

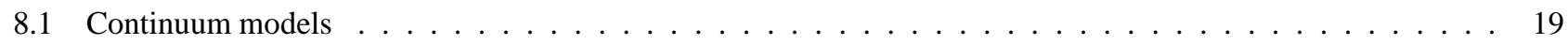

8.1.1 The Initiation of Angiogenesis . . . . . . . . . . . . . . . . . . . . 20

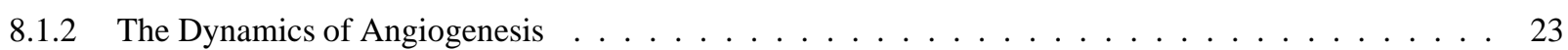

8.1 .3 Mechanochemical Models . . . . . . . . . . . . . . . . . . . . . . . . 29

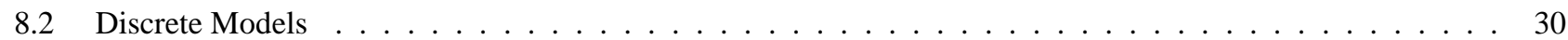

9 Summary and remarks 32

10 Appendix: Glossary of Selected Terms and Abbreviations 37

References $\quad 39$ 


\section{Introduction}

Cancer is a collection of diseases with the common feature of uncontrolled cellular growth. Most tissues in the body can give rise to cancer, some even yield several types, and each cancer has unique features. The salient feature of cancer cells is that the mechanisms that control growth, proliferation and death of cells in a multicellular organism are disrupted, often as a result of mutations. The $\sim 10^{13}$ cells in the human body are subject to numerous checks and balances that, to varying degrees, are absent, ignored or affirmatively avoided during cancer development. In effect, cancer cells escape the usual controls on cell proliferation and proliferate excessively to form a neoplastic growth or tumor.

Initially, solid tumors are avascular i.e., they do not have their own blood supply, and rely on diffusion from nearby vessels to supply oxygen and nutrients and to remove waste products. As the tumor grows nutrient demand increases until the flux of nutrients through the surface of the tumor is too small to supply the entire mass of cells. A necrotic core of dead cells develops at the center and eventually the tumor stops growing and reaches a steady state size of $\sim 1-3 \mathrm{~mm}$, in which the number of dying cells counterbalances the number of proliferating cells. Growth can resume only if the tumor becomes vascularized i.e., if it becomes permeated with a network of capillaries. An early response of tumor cells to hypoxia (oxygen deprivation) is the expression of genes that code for signaling molecules (growth factors, primarily vascular endothelial growth factor (VEGF) and basic fibroblast growth factor (bFGF; also called FGF-2) ${ }^{1}$ that are used to induce a nearby vessel to grow new capillaries to vascularize the tumor through a process called angiogenesis. These growth factors diffuse from tumor cells to the nearby primary vessels, and initiate a cascade of processes, including the activation of endothelial cells (ECs) that line the blood vessel walls, inducing them to proliferate and migrate chemotactically towards the tumor. This results in the creation of a new capillary network that extends from a primary vessel into the growth-factor-secreting tumor, thereby bringing essential nutrients to the tumor and providing a shorter route for the spread of cancer cells to other parts of the body (Folkman, 1971). Thus angiogenesis is the sine qua non for cancer invasion, and understanding the mechanisms that control it will provide the basis for rational therapeutic intervention. Angiogenesis involves many intermediate steps, and to give the reader a brief overview of the entire process, we summarize some of the main steps involved and illustrate them schematically in Figure 1. Each of these steps will be discussed in greater detail in later sections.

1. Activation of Endothelial cells by Angiogenic Growth Factors. A hypoxic tumor produces and releases growth factors. These are commonly referred to as tumor angiogenic factors, or TAFs (examples are VEGF and bFGF). The TAFs diffuse into the nearby tissue and bind to receptors in the membrane of endothelial cells lining a nearby primary blood vessel. Occupancy of these receptors triggers an intracellular signal transduction cascade that leads to gene transcription and production of factors needed for later steps, as well as changes in the mechanical structure of the cell. This switches the endothelial cell (EC) from the quiescent endothelial phenotype that prevails in an established vessel to an activated mesenchymal phenotype, which secretes enzymes and growth factors and can migrate in response to TAFs and proliferate.

2. Migration and Proliferation of ECs. An early response of ECs following activation is the secretion of matrix metalloproteinases (MMPs), which are enzymes that degrade the basement membrane surrounding the vessel and the adjacent extracellular matrix (ECM). This may in turn also release sequestered growth factors from the ECM. Degradation of the ECM facilitates migration of ECs toward the tumor, guided by a chemotactic response to the TAFs. The ECs may attach to each other, form a hollow tube-like cavity (the lumen) in their center and thereby begin a new capillary whose growth is oriented toward the tumor. After the ECs have left the primary vessel and migrated some distance into the ECM they begin to divide, and the daughter cells are added to the growing capillary.

3. Vessel Maturation. In a later stage these new capillaries are stabilized by smooth muscle cells and pericytes that envelop the capillaries and provide structural stability. Nearby sprouts of new capillaries may sometimes form loops that create a small bypass in the primary vessel, but if they reach the tumor and form a connected loop, blood flow can supply the tumor with nutrients and provide a shorter path to the systemic vasculature.

Angiogenesis is crucial to tumor growth, but it is not unique to that process: formation of a functional vascular network occurs during embryogenesis and later in growing tissues. Vascularization occurs by two distinct processes: vasculogenesis, which occurs only during early embryogenesis and produces a largely-unstructured capillary bed, and angiogenesis, which refines this basic network and produces the complex system of large and small vessels that permeate a tissue (Yancopoulos et al., 2000; Papetti \& Herman, 2002). Under normal physiological conditions angiogenesis is regulated by a balance between angiogenesis-promoting factors such as TAFs and factors that inhibit this process. In normal tissue, angiogenesis is largely absent, except in the ovary, throughout the menstrual cycle, during wound healing, and during placenta formation. However, it does occur under a variety of pathological conditions, such as diabetic retinopathy, arthritis, and chronic inflammation (Muthukkaruppan et al., 1982; Folkman, 1995a). Once tumor angiogenesis succeeds, new capillaries supply it with the

\footnotetext{
${ }^{1} \mathrm{~A}$ glossary of frequently-used terms is included at the end.
} 
nutrients needed for continued growth (Folkman, 1986; Muthukkaruppan et al., 1982). The tumor can then expand rapidly, and soon may reach several $\mathrm{mm}^{3}$ in size and contain a large number of cells $\left(\sim 10^{9}\right.$ cells) (Folkman et al., 1995).

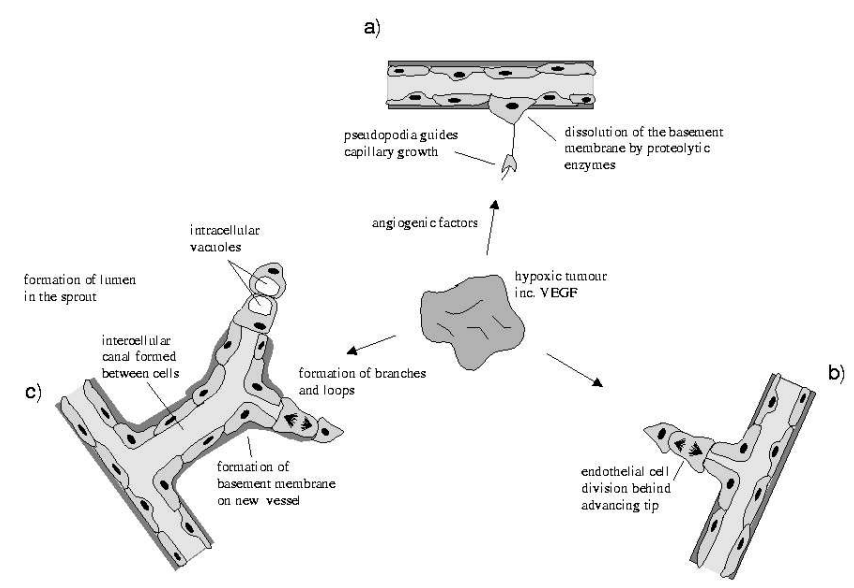

Figure 1: A schematic of the major steps involved in angiogenesis. (a) ECs on the primary blood vessel are activated by growth factors produced by the tumor. These cells loosen contacts with their neighbouring ECs, extend their leading edges to form pseudopodia in the direction of migration and secrete proteolytic enzymes that locally degrade the supporting ECM, initiating the migration of ECs towards the tumor. (b) Further sprout extension occurs when ECs behind the tip of the advancing sprout begin to proliferate. The cells continue to make their way through the ECM towards the angiogenic stimulus. In (c), some of the ECs in the sprout start to reassociate with each other, and develop intercellular and intracellular lumen, leading to the formation of tube-like structures. Branches form when the sprout tip splits into two, whereby an intracellular vacuole in the tip cell becomes Y-or T-shaped. As the vessels mature, the ECs resynthesize a basement membrane to restore continuity. Closed loops are formed when neighbouring sprouts fuse together, a process called anastomosis, and this marks the onset of blood circulation in the new network.$$
\text { endothelial cells that form capillary tips migrate up a gradient of }
$$

Tumor-induced angiogenesis provides the crucial link between the avascular phase of solid tumor growth and the more harmful vascular phase. In the latter phase, tumor cells far removed from a primary vessel and hence the systemic circulation can enter the circulatory system via the tumor vasculature, which is significantly more permeable than mature veins and arteries (Dvorak et al., 1995). This enables the tumor cells to migrate to tissues in other parts of the body and form secondary masses, called metastases (Ruoslahti, 1996; Liotta \& Stetler-Stevenson, 1993). This invasiveness makes many cancers very lethal (exceptions include brain tumors, which do not cross the blood-brain barrier), for without angiogenesis a growing tumor remains benign and easily controllable, and a complete cure can usually be achieved by removing the tumor. However a cancer that has metastasized often colonizes so many tissues that a cure by surgery alone becomes impossible. Thus, acquiring the ability to emigrate to another tissue is a key event in the development of a cancer (Liotta \& StetlerStevenson, 1993).

The aim of this review is to identify aspects of the angiogenic process for which mathematical models can provide significant new insights. Models for the growth of the tumor itself have been addressed in the monograph by Adam \& Bellomo (1997). To give a flavor of what has been done in regard to tumor-induced angiogenesis we describe some of the models briefly; details are given later. Much of the mathematical modeling has focused on the way in which tumor angiogenic factors initiate and coordinate capillary growth. For example, Orme \& Chaplain (1996) and Levine et al. (2000) have developed continuum models for initiation and outgrowth of buds from a primary vessel. These models postulate that the vascular endothelial cells that form capillary tips migrate up a gradient of angiogenic factor released by the tumor. The density of new capillaries as well as the concentrations of angiogenic factors evolve according to coupled nonlinear partial differential equations that will be described later. Others have used continnum models to study the interactions between endothelial cells and the extracellular matrix during angiogenesis in order to understand how cells respond to not only chemical signals via chemotaxis, but also to mechanical signals via haptotaxis (Olsen et al., 1997; Pettet et al., 1996; Anderson \& Chaplain, 1998b).

Discrete models that treat cells as individual units have also been developed to model angiogenesis (Anderson \& Chaplain, 1998b; Stokes \& Lauffenburger, 1991). In contrast with the continuum models, discrete models can track individual cells and can incorporate more details about cell movement and interaction with the tissue. The results of the discrete model agree with the predictions of the continuum model and in addition are able to produce capillary networks with structure and morphology similar to those observed in vitro. A discrete model that incorporates rules for sprout branching and anastomses and also contains an element of stochasticity for the movement of the cells has also been proposed(Anderson \& Chaplain, 1998b).

Our understanding of the basic microscopic and macroscopic processes involved in angiogenesis is growing rapidly and for many of these processes enough is now known to warrant mathematical models that incorporate detailed biochemical and physiological information. As a result, significant progress toward an integrated quantitative model of angiogenesis, and computational tools to explore the effects of various changes in the constituent subprocesses, is achievable in the near future. Our goal herein is to provide both the necessary background biological material, and an entree to the current literature on mathematical models of angiogenesis, to facilitate entry into this area by other researchers. As in much of biology, the current data is sometimes contradictory, and our summaries of important observations should be viewed as a starting point, not a 
definitive statement of facts.

In view of what has been said it is clear that ECs play a major role in the angiogenic process. We therefore organize the review of the biological background around the angiogeneic events that are driven by the ECs. We start by reviewing normal vessel morphology and the signals involved in the early changes within the parent vessel during angiogenesis. We then follow the route of the endothelial cells from the parent vessel, as they cross the extracellular matrix leaving behind the branched and looped EC structures on their way to the tumor, and we then discuss the formation and maturation of the new vessel. We finish the first part of this review by highlighting some of the current knowledge on angiogenesis inhibitors and new anti-angiogenesis therapeutic strategies. More comprehensive reviews of the biology can be found in $e$.g., Paweletz \& Knierim (1989); Carmeliet \& Jain (2000), and introductory accounts to the entire field can be found in a special issue of Scientific American (275, \# 3, Sept (1996)) and (Bicknell et al., 1997).

\section{Normal Vascular Physiology}

\subsection{Blood Vessels}

The vasculature is one of the main organs in the body, extending more than $900 \mathrm{~m}^{2}$ (Griffioen \& Molema, 2000). The structure of blood vessels, which are composed of three cell types, is relatively simple (Figure 2).

Endothelial cells are in direct contact with the blood and form an inner luminal lining in every blood vessel. Sub-endotheliallylocated pericytes and smooth muscle cells are embedded within an elastic lamina or basement membrane (BM) which surrounds the endothelium, and fibroblasts form part of an outer layer. The remainder of the outer layer, called the adventitia, is made up of a thin layer of connective tissue (or extracellular matrix, ECM) that is continuous with that of the surrounding tissue. Depending on the location in the body, the vessels' diameter and function, and the amounts of BM and ECM of the vasculature, its phenotype and composition may vary. For instance, in the finest branches of the vascular tree, such as capillaries and sinusoids, the vessels consist of just ECs and a basal lamina, together with a few scattered pericytes (Alberts et al., 2002).

Vascular ECs play a major role in several regulatory processes in the body (Griffioen \& Molema, 2000). In most adult tissues ECs proliferate very slowly and have a lifetime ranging from months to years. However, during vessel repair or angiogenesis ECs proliferate much more rapidly, with a doubling time of just a few days (Alberts et al., 2002). In addition to their role in vascular remodeling, ECs can direct cells from the immune system to lymphoid organs or inflammatory sites through the expression of specific cellular adhesion molecules (such as E-selectin and intercellular adhesion molecule-1) and soluble factors, such as chemoattractants, cytokines and chemokines. They are also selectively permeable for small peptides and pro-

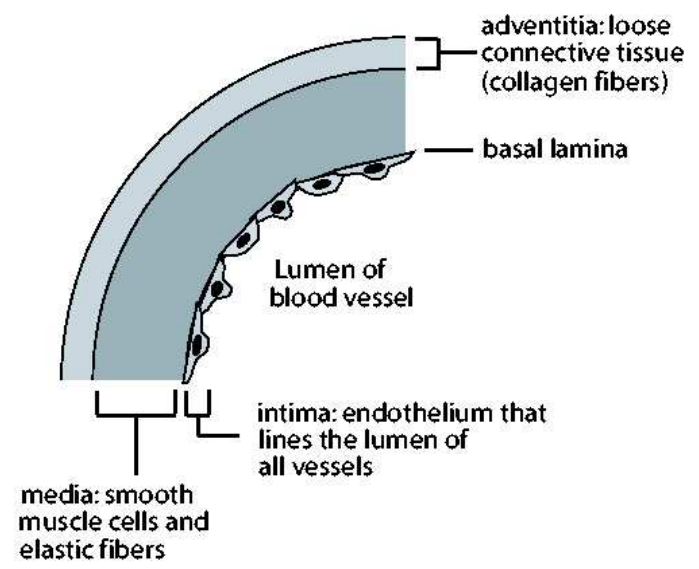

Figure 2: A schematic diagram showing the three layers that make up the wall of a blood vessel. The inner layer comprises a one-cell-thick sheet of endothelial cells, called the endothelium, and a thin layer of spongy connective tissue that forms the basement membrane for the endothelium. Together these are sometimes known as the tunica intima. The central layer, the tunica media, is primarily smooth muscle and elastic fibers and is usually the thickest. The outermost layer, which attaches the vessel to the surrounding tissue, is called the tunica adventitia, and is made up of connective tissue with varying amounts of elastic and collagenous fibers.

teins, and also regulate blood coagulation via the synthesis of thrombomodulin, tissue factor (TF) and tissue-type plasminogen activator (t-PA) (Griffioen \& Molema, 2000).

A first step in the activation of vascular ECs in angiogenesis involves weakening of cell-cell junctions, via the downregulation of E-cadherin, which is a cell-cell adheren junctional protein. Once freed from contacts in the primary vessel, ECs must respond chemotactically by organizing their motile machinery to produce force in the proper direction, degrade the basement membrane locally, and begin migration.

\subsection{Extracellular Matrix}

To gain traction to move, ECs adhere to fibers in the ECM via adhesive molecules called integrins, and they communicate with each other via other adhesive molecules, such as vascular endothelial (VE) cadherin (Breier, 2000a). In response to angiogenic growth factors, ECs up-regulate the expression of several integrin receptors and migrate into the ECM. The migrating ECs 
clear a path through the ECM by secreting proteases, and several of the matrix metalloproteinases proteinases (MMPs), either secreted or membrane-bound, and the plasminogen activator (PA)/plasmin system play a central role in the local proteolytic remodeling of matrix proteins and migration of ECs. The deposition of provisional ECM components is also important for EC migration and survival during angiogenesis.

The ECM is comprised of three main types of molecules: (i) structural proteins such as collagen and elastin, (ii) specialized proteins such as fibrillin, fibronectin, vitronectin and laminin, and (iii) proteoglycans, which consist of a protein core surrounded by attached long chains of repeating units. Collagens, which occur in at least a dozen forms, provide tensile strength and elasticity to the ECM. Types I, II, \& IV are the most abundant: Type I forms about $90 \%$ of the collagen, Type II makes up the major fibers of cartilage, and Type IV collagen molecules make thin supportive mats that underlie cell sheets and tubes (Alberts et al., 2002). Fibronectins are large glycoproteins which are assembled from two fibrous polypeptides; they are found in basal laminae and in the loose connective tissue underneath the skin and between the body organs. Laminin is a large cross-shaped glycoprotein which is constructed from three polypeptides. Laminin has binding sites for the type IV collagen of basal laminae at the tips of two arms of the cross. These molecules are primarily linkers in networks and anchors that attach cells to basal laminae. In vertebrates proteoglycans occur wherever collagens occur in the ECM. Individual proteoglycans can link to collagen fibers, thereby forming the fiber-network complex of the ECM. Proteoglycans also function as sites for cell adhesions, both temporary and permanent.

The basement membranes that surround many vessels are a thin sheet-like structure of ECM components, primarily laminins, type IV collagen (the main component) and heparin sulphate proteoglycans, that serves as a support and barrier in many multicellular organisms. Localized degradation of the basement membrane requires the expression of proteases that are specific for the resident ECM, and the concomitant expression of protease inhibitors to prevent excess matrix degradation and loss of tissue integrity. Two MMPs (MMP-2: gelatinase A and MMP-9: gelatinase B) have very high enzymatic activities when acting on type IV collagen.

Next we describe the compounds involved in vascular EC activation and the EC-derived proteases that dissolve the ECM, thereby facilitating EC migration and subsequent vessel formation.

\section{Early Events in Angiogenesis}

\subsection{Signaling From Tumor To Vessel at the Onset of Angiogenesis}

Signaling molecules such as soluble growth factors and cytokines coordinate multiple cellular responses during angiogenesis, ranging from induction of cell proliferation and new gene expression to directed cell migration and modulation of cell-cell interactions. Recent studies in a variety of tumors have shown that tumor cells stimulated by hypoxia start secreting some of these angiogenic factors. These factors stimulate EC growth and migration, and have different effects on EC expression of proteases that degrade the ECM, such as the plasminogen activator (PA)-plasmin system and the MMP family components (discussed below). The best known of these growth factors are transforming growth factor beta (TGF- $\beta$ ), basic fibroblast growth factor (bFGF) and vascular endothelial growth factor (VEGF), which is also called vascular permeability factor (VPF). VEGF is thought to be the most potent angiogenic inducer, because it is largely EC-specific, while FGF can stimulate the proliferation of other cell types as well. These factors activate specific cell surface receptors, which in turn activate intracellular signal transduction pathways. We discuss the effects of these growth factors next; discussions of other angiogenic factors can be found in Bouck et al. (1996); Folkman (1995a); Folkman (1995b) and Liekens et al. (2001).

\subsubsection{Transforming Growth Factor Beta (TGF- $\beta$ )}

Transforming growth factor $\beta$ is produced by many cell types, including platelets (Assoian. et al., 1983), ECs and pericytes (Sato \& Rifkin, 1988; Sato \& Rifkin, 1989), fibroblasts (Lawrence et al., 1984), and transformed cells (Pennington et al., 1991), and has been isolated from a number of tissue sources (Assoian. et al., 1983; Frolik et al., 1983; Roberts et al., 1986). TGF- $\beta$ is a member of the growth factor family that acts on some cells to stimulate cell proliferation and others to inhibit it, or stimulates at one concentration and inhibits at another. For example, TGF- $\beta$ is a strong angiogenic factor in the cornea (Folkman \& Klagsbrun, 1987) and at subcutaneous sites in newborn mice (Roberts et al., 1986), although TGF- $\beta$ blocks the migration and growth of vascular ECs (Frater-Schroeder et al., 1986). TGF- $\beta$ is released in its latent form (LTGF- $\beta$ ) and in this state is unable to bind to the TGF- $\beta$ receptor (Pircher et al., 1986; Andres et al., 1989). However, LTGF- $\beta$ can be converted into the active form by the protease plasmin (Lyons et al., 1988) (see Section 3.2.1). Interestingly, active TGF- $\beta$ has been found to induce the synthesis of plasminogen activator inhibitor 1 (PAI-1; described later), which blocks the conversion of plasminogen to plasmin (Sato et al., 1990; Pepper \& Montesano, 1990). Thus, the activation of LTGF- $\beta$ is a self-regulating system, since plasmin formation increase leads to enhanced conversion of LTGF- $\beta$ to TGF- $\beta$. However, more TGF- $\beta$ induces PAI-1 synthesis, thereby reducing plasmin formation, and with less plasmin, conversion of LTGF- $\beta$ to TGF- $\beta$ decreases and PAI-1 synthesis will fall, which allows plasmin formation to increase and the cycle starts over again (Figure 3 ). 


\subsubsection{Basic Fibroblast Growth Factor (bFGF)}

bFGF is a multi-functional growth factor present in several tissues in vivo and is synthesized by a number of cell types in vitro (Gospodarowicz et al., 1987; Moscatelli et al., 1986). Together with VEGF, bFGF is one of the most commonly identified growth factors in tumors (Folkman et al., 1995). It is a potent endothelial growth factor in vitro, and is thought to function as an autocrine factor in cultures of ECs (Sato \& Rifkin, 1988; Saksela \& Rifkin, 1990). When released by cells, bFGF binds to heparin sulfate proteoglycans with high affinity (Rifkin \& Moscatelli, 1989). Heparin sulfate is an abundant component of the ECM and is synthesized as a cell-membrane-associated heparin sulfate proteoglycan (HSPG) or as an ECM-associated HSPG. The uncomplexed bFGF is readily degraded by various proteinases, but bFGF bound to HSPG is protected from proteolytic inactivation (Saksela et al., 1998). bFGF-HSPG complexes on the cell surface are quickly internalized and degraded, but matrix-bound bFGF persists as a source of growth factor (Moscatelli \& Rifkin, 1988; Flaumenhaft et al., 1989), and matrix-bound bFGF may be released by plasmin. This release of growth factor is particularly important since it raises the level of soluble bFGF locally. Soluble bFGF-HSPG complexes do not bind to the matrix-binding sites as does the uncomplexed bFGF. Thus, complex formation with heparin sulfate and its subsequent release by plasmin would facilitate the diffusion of the growth factor in the tissue to interact with bFGF receptors on the cell surface. There are at least four such high-affinity tyrosine kinase receptors for bFGF, abbreviated FGFRs (Johnson \& Williams, 1993). Downstream effects of FGFR binding include EC cell proliferation and migration, protease production, and differentiation (Schlessinger et al., 1995).

\subsubsection{Vascular Endothelial Growth Factor (VEGF)}

VEGF was initially defined by its ability to induce vascular leak and permeability, as well as for its ability to promote vascular EC proliferation (Yancopoulos et al., 2000). For this reason it was originally termed vascular permeability factor. VEGF is not produced by ECs, but it is secreted in large quantities by tumor cells, and this secretion is stimulated by hypoxia. VEGF can also be synthesized by other cell types including macrophages and mast cells (Weninger et al., 1996), and there is evidence that VEGF and FGF function synergistically (Pepper et al., 1992; Goto et al., 1993). In the circulation the half-life of VEGF is approximately 3 min (Folkman, 1995a), but the half-life in a tissue is not known. VEGF potently increases the permeability of the vascular venules, and microvascular hyperpermeablility is one of the earliest steps in angiogenesis. This leads to extravasation (effusion) of fibrinogen and other plasma proteins, followed by the deposition into the extracellular space of a fibrin gel, which acts as a 'pro-angiogenic' matrix that supports the inward migration of new blood vessels (Balsari et al., 1999). However, recent findings have shown that enhanced vascular permeability is not a requirement for VEGF-dependent angiogenesis (Eliceiri et al., 1999), and therefore VEGF-induced protein extravasation does not appear to be a major component of the angiogenic process.

The cellular responses to the members of the VEGF family are mediated primarily by the high affinity EC receptors VEGF receptor-1 (VEGFR-1) and VEGF receptor-2 (VEGFR-2; also called Flt-1). VEGFR-2 is considered the main signaling VEGF receptor in ECs (Breier, 2000b), whereas VEGFR-1 may have a negative role either by acting as a decoy receptor or by suppressing signaling through VEGFR-2 (Yancopoulos et al., 2000). Both receptors are expressed to a lesser extent on monocytes/macrophages and some tumor cell types (Griffioen \& Molema, 2000). Studies have shown that the expression of VEGF-1 and -2 is upregulated under hypoxic conditions (Kremer et al., 1997; Shen et al., 1998). Furthermore, VEGFR-2deficient mice fail to develop a vasculature and have very few ECs, whereas mice lacking VEGFR-1 have excess EC formation which abnormally coalesce into disorganized tubules (Fong et al., 1995). 
There are several members in the VEGF gene family, including VEGF, placenta growth factor, and VEGF -B, -C and -D (Veikkola \& Alitalo, 1999; Ferrara, 2000). VEGF, also designated as VEGF-A, is the major regulator of normal and abnormal angiogenesis, including that associated with tumors (Kim et al., 1993; Aiello et al., 1995). So far, six isoforms of VEGF-A have been found, resulting from alternative splicing from a single VEGF-A gene. These are VEGF ${ }_{121,145}$, 165,183, 189 and $\mathrm{VEGF}_{205}$ (Tischer et al., 1991; Houck et al., 1991). When expressed in a human embryonic kidney cell line, $\mathrm{VEGF}_{121}$, the shortest form, was secreted and freely soluble in the tissue culture medium. $\mathrm{VEGF}_{189}$ was secreted, but was almost entirely bound to the cell surface or the extracellular matrix, and the transcript of $\mathrm{VEGF}_{165}$, the most common form of VEGF, displayed an intermediate behavior (Houck et al., 1992). Release of the ECM bound forms of VEGF 165 and VEGF $_{189}$ by treatment with heparin sulfate suggests that the binding sites for these VEGF varients in the cell culture involves a heparin-containing binding site, similar to the binding domain for bFGF (Houck et al., 1992). Thus, binding of the long forms of VEGF to heparin binding domains in the ECM could provide a reservoir of biologically-active VEGF available to ECs following its release. As with bFGF, the serine protease plasmin is a potential key protease involved in the release of the matrix-bound VEGF varients into biologically-active soluble forms (Poltorak et al., 2000; Neufeld et al., 1999). Plasmin may thus serve to liberate the angiogenic factors $\mathrm{VEGF}_{165}$ and $\mathrm{VEGF}_{189}$ as well as bFGF from the extracellular matrix (Figure 3).

VEGF has a number of important effects in angiogenesis. It induces a cascade of phosphorylations including the mitogenactivated protein kinase (MAPK), PLC, GAP, the RAS GTPase-activating protein. Through PLC activation and subsequent $\mathrm{IP}_{3}$ production, it stimulates transient elevations of $\mathrm{Ca}^{2}{ }^{+}$lasting $\sim 30$ minutes. It also induces endothelial cell migration by increasing plasmin activator (PA) synthesis (see below), and by increasing microvascular permeability through enhancment of the functional activity of vesicular-vacuolar organelles (VVOs). The mechanism of this increase of functional activity of VVOs is unknown, but it is likely that these organelles facilitate the secretion of plasma proteins to the tissue. These proteins are proteolytic enzymes that degrade their basal lamina and the intercellular matrix Paweletz \& Knierim (1989). As a result, the migration of EC towards the tumor is facilitated. There is also evidence that VEGF is an important survival factor for the tumor vasculature (Gerber et al., 1999; Yuan et al., 1996), and induces the expression of the anti-apoptotic factors Bcl-2 and A1 in human ECs (Gerber et al., 1999). Survival rates of patients with VEGF-rich tumors is significantly poorer than that of patients with VEGF-poor tumors (Toi et al., 1995). Furthermore, inhibition of VEGF activity has been shown to suppress growth of a wide varity of tumor types in murine models (Ferrara \& Keyt, 1997; Ferrara, 1999).

In summary, much is known about how VEGF stimulates migration of ECs, particularly via alterations of the ECM. VEGF also stimulates EC proliferation by activating the MAPK pathway, but in the initial stages of neovascularization proliferation of ECs does not seem to be important. However, in the later stages of the angiogenic process EC proliferation appears to be a key event; vessels cannot reach the target tumor without EC proliferation (Sholley et al., 1984). For more details on the receptors, biology, and function of VEGF see Brown et al. (1997); Ferrara \& Keyt (1997) and Christofori (1996).

\subsection{Modifications of the ECM that Facilitate EC Migration}

An increase in growth factors such as VEGF leads to secretion of proteolytic enzymes that degrade the ECM by ECs, monocytes/macrophages and by the tumor cells themselves (Seandel et al., 2001). Many of the proteases implicated in angiogenesis belong to two general classes: matrix metalloproteinases (MMPs), which depend on $\mathrm{Ca}^{2+}$ or $\mathrm{Zn}^{2+}$ for activity; and serine proteases, which contain components of the PA-plasmin system. Together, these proteases cooperate to degrade ECM components such as collagen, laminin and fibronectin. However, degradation of the ECM is not done in shotgun fashion: selective cleaving of ECM molecules can release growth factors or inhibitors, and by exposing cryptic binding sites for adhesion and destroying others, can dramatically alter the invasive ability of the cells. There is also a third class of enzymes called cysteine proteases, which are mainly involved in the intracellular degradation of phagocytosed material, but their involvement in angiogenesis is unclear (Tamada et al., 2000). Increased activity of PA-plasmin components and MMPs has been well documented in angiogenesis, and we discuss these next.

\subsubsection{The PA-Plasmin System}

The central component of the PA-plasmin system is plasmin, which is formed from the proteolytic cleavage of its zymogen plasminogen (Andreasen et al., 1997). Plasminogen is an inactive serine protease precursor that is abundant in the bloodstream at a concentration of about $1.5 \mu \mathrm{M}$, and accumulates at sites of tissue remodeling such as wounds, tumors, and sites of inflammation. Urokinase- and tissue-type PAs (uPA and tPA) are the principal activators of plasminogen. Like plasmin, uPA and tPA are serine proteases, but in contrast to plasmin, they have very restricted substrate specificity (principally plasminogen). Plasmin degrades several ECM components including fibronectin, laminin and the protein core of proteoglycans. It does not degrade elastin and collagens, but it can degrade gelatin, the partially degraded or denatured forms of collagen (Mignatti \& Rifkin, 1996). There is general agreement that the primary role of tPA is to generate plasmin for thrombolysis (the breaking up of a blood clot), while uPA generates plasmin involved in degradation of ECM. The plasminogen activation system is tightly regulated by potent inhibitors, which are members of the serine protease inhibitors superfamily, called serpins. When 
not bound to fibrin or specific cell surface plasmin(ogen) receptors, plasmin is rapidly inhibited by $\alpha_{2}$-antiplasmin. The major extracellular inhibitor of both UPA and tPA is the serine protease inhibitor called plasminogen activator inhibitor (PAI) type-1. ECs secrete high levels of PAI-1s both in vivo and in vitro.

uPA is secreted as a soluble inactive precursor (pro-uPA) and binds with high affinity to a specific cell surface uPA receptor (uPAR, also termed GPI-group cell surface receptor) which is present on a variety of cells. When uPA binds with UPAR, it is activated and remains active on the cell surface for several hours. Human ECs in vitro contain about 140,000 uPARs per cell (Koolwijk et al., 1998). However, because many cells that synthesize urokinase and uPAR also express PAI-1, uPA:uPAR complexes are likely to be present only transiently on cell surfaces in vivo. Regulation of plasminogen activity also occurs by cellular receptors, directing the plasminogen to focal areas on the cell surface. These high affinity binding sites for plasminogen are found on various types of cells including ECs (typically 3.9 to $14 \times 10^{5}$ receptor molecules per cell). Thus, interaction of uPA with uPAR in the plasma membrane has three important consequences: (1) enzyme activity is localized to focal contact sites; (2) co-localization of uPA and plasminogen on the cell surface increases the efficiency of plasminogen activation and subsequent plasmin-dependent proteolysis, and (3) after complex formation with PAI-1, uPA is internalized together with the uPA receptor, followed by the degradation of the uPA:PAI-1 complex and the return of the unoccupied uPA receptor to the plasma membrane.

The uPA receptor has been found in cellular protrusions involved in cell migration and invasion (Werb, 1997). For example, in a human breast cancer cell line invading a reconstituted basement membrane, cell surface uPAR was found both at the leading edge of the cells and at pseudopodia penetrating into the membrane. Furthermore, it has been found recently that the UPA receptor may have an additional role: a UPAR occupied by uPA interacts avidly with the matrix-like form of vitronectin. Hence uPA, which promotes UPAR attachment to vitronectin, also provides a means of detachment by either forming complexes with PAI-1 or activating plasmin, which disrupts the interaction between uPAR and vitronectin by cleaving the uPAR:VN interaction site. Thus, the effects of uPA on cell migration may be caused by a proteolytic effectgeneration of plasmin at focal adhesion sites, catalyzed by uPAR bound UPA and leading to ECM degradation- as well as a non-proteolytic effect via stimulation of cell migration by enhancing UPAR:VN binding and hence adhesion at the leading edge. It has been suggested that both these mechanisms operate in vivo in some tumors, but the relative contribution of each to cell migration is not known.

The expression of both uPA and its receptor is increased by growth factors VEGF and bFGF, and this induction is regulated independently of their effects on cell proliferation (Koolwijk et al., 1998; Presta et al., 1989; Mignatti \& Rifkin, 1996). Note that although VEGF has a net effect to increase endothelial cell proteolytic activity, in some cell types it induces both uPA and PAI-1; evidence also indicates that bFGF and TGF- $\beta$ may induce co-expression of uPA and PAI-1 (Pepper et al., 1996). However, the magnitude of uPA and PAI-1 induction by these growth factors is markedly different. For example, in response to VEGF or bFGF, the uPA:PAI-1 ratio is significantly increased in favour of uPA, and so the net balance of proteolytic activity is positive, whereas TGF- $\beta$ has a net antiproteolytic effect. The co-induction of uPA and PAI- 1 is likely to provide a mechanism for preserving the integrity within the ECM by limiting matrix degradation to the pericellular environment. For example, in wound healing one of the roles for PAI-1 is to prevent premature dissolution of the fibrin clot, which provides a temporary scaffold for invading inflammatory cells (Pepper \& Montesano, 1990).

\subsubsection{The MMP Family of Proteinases}

The involvement of the MMP system in the process of angiogenesis has been demonstrated both in vitro and in vivo using MMP-specific inhibitors (Koolwijk et al., 1998). There are MMP family members with enzymatic activity against virtually all components of the ECM and basement membranes. Most importantly, the MMP family includes the only enzymes capable of cleaving fibrillar collagens. The MMPs can be divided into five groups on the basis of their substrate specificity: Collagenases, which can degrade intact collagen; Gelatinases, which can degrade collagen type IV and unfolded collagens; Stromelysins, which have a broad substrate specificity and can activate other MMPs; MT-MMPs, which play a role in the activation of gelatinase A; and other MMPs that differ in substrate specificity from the other MMP groups. For full details of these enzyme groups see (Koolwijk et al., 1998) and (Coussens \& Werb, 1996). MMP family members share the common feature that they bind zinc at their active site and are dependent on calcium for their catalytic activity, and for this reason they are characterized as metal-binding proteinases. All MMPs are synthesized as inactive precursors and secreted as soluble enzymes, with the exception of the membrane-type MMPs (MT-MMPs), which contain a transmembrane domain and are embedded in the cell surface. It is not known how MMPs are activated in vivo, but in vitro studies have shown that serine proteases, such as plasmin or uPA, elastase and trypsin, cleave propeptide domains of secreted pro-MMPs (except pro-gelatinase A), which yields a truncated and catalytically active enzyme. Also, some of the activated MMPs can further activate other proMMPs. For example, stromelysin-1 (MMP-3) activates pro-collagenase 1 and Pro-gelatinase B (Coussens \& Werb, 1996). A schematic of the steps involved in activation is shown in Figure 4. 
Cell-mediated activation mechanisms are also possible. Recently, it has been shown that MMP-2 can be activated by the membrane-type MMPs both in vitro and in vivo (Koolwijk et al., 1998). The MTMMPs themselves are activated intracellularly by a group of calcium-dependent transmembrane serine proteinases (termed furin/PACE/kex-2-like proteinases). In addition to activating MMP-2, MT-MMP can serve as a MMP-2 receptor (Nakahara et al., 1997) and thus could be involved in the recruitment of the MT-MMP/MMP-2 complex to sites of invasion. MT-MMP may also degrade ECM. Thus, it is possible that MT-MMP functions both in MMP-2 activation and in localizing such proteases to sites of cell invasion. Recent studies showed that when membrane type-1 MMP was overexpressed in a human melanoma cell line (RPM17951), the cells made contact with the ECM, activated soluble and ECM bound MMP-2, and degraded and invaded the ECM (Nakahara et al., 1997). In this study the membrane-bound protease was predominantly localized at surface extensions called invadopodia Overexpression of MT-MMP without invadopodial localization caused activation of soluble MMP-2, but did not facilitate ECM degradation or cell invasiveness.

\section{Construction of a Growing Vessel}

Cell migration plays a central role in embryogenesis and wound healing, and in angiogenesis it leads to invasion and capillary formation. Outgrowth of a sprout requires a supply of ECs and their proper orientation and adhesion. In early stages of outgrowth the vessel wall and perhaps cir-

culating EC precursors (the monocytes) supply the necessary cells, but in later stages cell proliferation is essential; without it sprouts eventually regress (Ausprunk \& Folkman, 1977). Activated ECs exhibit chemotaxis toward growth factors and perhaps other cytokines released by the tumor cells, by macrophages, or even other types such as mast cells (Norrby, 2002). Effective chemotaxis is a complex and as yet poorly-understood process that hinges on extracting directional information from an extracellular scalar field (the concentration of attractant) and organizing the intracellular motile machinery so as to exert force in the proper direction. We begin this section by describing some of the forces that act on a motile cell as it migrates through the ECM. We then describe how individual ECs re-associate and assemble into tubular structures to form capillary lumena. The role of adhesive cell-cell and cell-ECM interactions in lumen formation is also discussed. More comprehensive reviews of cell motility are given in (Lauffenburger \& Horwitz, 1996; Zhu et al., 2000; Franz et al., 2002).

\subsection{Cell Orientation to Extracellular Signals and Movement Through the ECM}

Two distinct steps are necessary in order that a cell can move toward the source of a chemoattractant: (i) it must orient itself properly and (ii) it must generate the necessary forces. Amoeboid cells such as ECs are large enough to detect gradients of attractants across their length, and in fact, some eukaryotic cells can respond to differences as small as $2 \%$ across their length, frequently at concentrations at which molecular fluctuations, and hence noise in the signal, are significant. Whereas the level of extracellular noise is not controllable, the intracellular noise level of the transduced signal may be far less, and small differences in extracellular signal may be amplified within the cell. Recent studies on the cellular slime mold Dictyostelium discoideum and on neutrophils show that the internal gradient of molecules such as activated phosphatidylinositol-3 kinase (PI3K) can be much higher than the external gradient of chemoattractant. This generates plecstrin homology (PH) sites on membrane-localized phospholipids such as $\mathrm{PI}(3,4,5) \mathrm{P}_{3}$ and the subsequent recruitment of $\mathrm{PH}$-containing $\mathrm{PI}(3,4,5) \mathrm{P}_{3}$ binding proteins to the plasma membrane, including the kinase Akt/PKB (Parent \& Devreotes, 1999; Servant et al., 2000). Akt/PKB itself becomes activated upon recruitment to the membrane and in Dictyostelium activates the serine/threonine kinase PAKa, which in turn regulates actin poplymerization, myosin II assembly, cortical tension, and retraction of the trailing tail of the cell (Funamoto et al., 2001). 
The forces needed for cell migration are generated by the actin cytoskeleton and by myosin. Motion is generally described as a multi-step process involving (i) actin polymerization at the leading edge, which leads to extension of a pseudopod or lamellipodia, (ii) attachment of the pseudopod or lamellipod to the substrate or ECM via integrin-mediated adhesion sites, (iii) contraction of the cell via actin-myosin contraction (Fukata et al., 2001), (iv) release of adhesion sites at the rear, and (v) recycling of adhesion receptors and other membrane components to the front of the cell via endocytosis and vesicular transport (Sheetz et al., 1999). When a cell is stimulated to move, GTPases in the Rho family are involved in transducing signals from surface receptors to downstream effectors such as the Arp $2 / 3$ complex that associate directly with actin to nucleate filament assembly (Higgs \& Pollard, 2001). Integrins transmit mechanical stresses between the actin cytoskelton of the cell and the ECM, primarily via binding to fibronectin, vitronectin, and laminin, and thereby have an important effect on cell shape. The speed and efficiency of migration is affected by many factors, including expression levels of adhesion receptors, secretion of matrix-modifying proteases, and properties of the ECM. The integrin $\alpha_{v} \beta_{3}$ is one of the primary integrins in EC, and it has been shown recently that they are selectively recruited to the leading edge of lamellipodia (Kiosses et al., 2001). This localization can promote localized adhesion and alter the overall strength of adhesion at the 'front' (the direction of motion) as opposed to the rear. A mechanism for activation of $\alpha_{v} \beta_{3}$ by VEGF signaling, via the activation of PI3K and its downstream effectors has been suggested by (Byzova et al., 2000).

\subsection{Capillary Lumen Formation}

In order to assemble into tubular structures, ECs must lose their invasive phenotype, reassociate with matrix proteins, and develop cell-cell contacts in a tubular conformation. Many in vitro studies have attempted to define the different morphological steps in the formation of capillary sprouts. A commonly used method of in vitro angiogenesis is the growth-factor-induced formation of cord-like structures when ECs are cultured on an extracellular matrix secreted by Engelbroth-Holm-Swarm sarcoma cells, commonly known as Matrigel (Yang et al., 1999). In these models, capillary-like lumens are demonstrated which closely mimic those observed within collagen matrices in vivo (Davis \& Camarillo, 1996). Two different types of capillary vessel formation can be distinguished which occur independently of each other or in combination within a single sprout: sprout formation through intracellular or intercellular lumen formation.

\subsubsection{Intracellular Lumen}

An intracellular lumen develops through the formation of intracellular vacuoles that enlarge and coalesce in response to EC contact with collagen matrix. Vacuole formation occurs through a pinocytic process with internalization of plasma membrane and molecules from the extracellular space. The union of adjacent cellular lumens results in the formation of a continuous capillary tube, which traverses the cytoplasm of the cells, or the nucleus, so that the nucleus sometimes seems to be split into two parts. Early in their development, the tubes are filled with a partially fibrillar, partially membranous material. Later the material dissolves, or is cleared from the lumen in some way, and the tubes become hollow as the capillary network matures. In a study by Yang et al. (1999), the time course of endothelial vacuole and tube formation was investigated in a 3D collagen lattice cell culture with a mixture of bFGF and VEGF. As early as 2 hours, small vacuole-like structures could be observed in some ECs. The frequency of vacuole formation increased and at 8 hours virtually all cells contained these vacuoles. In these cells the small vacuoles appeared to fuse to form large vacuole structures, and at 48 hours these appeared as interconnecting lumens, formed either by individual cells aligned end to end or, in some cases, by several cells surrounding a single lumen. Under these conditions, the ECs rapidly assemble into a branched interconnected network of tube-like structures which is almost identical to capillary vascular beds in vivo. A branch in this type of lumen originates within a single cell, whereby the cylindrical vacuole within a cell forms a ' $\mathrm{Y}$ ' or a ' $\mathrm{T}$ ', each arm of which aligns with the vacuole of another cell. After the branch has matured, the original EC can usually be found at the intersection of the three tubes (Folkman \& Haudenschild, 1980). The formation of intracellular lumina is not specifc to endothelia but can also be observed in some tumor cells and fibroblasts (Konerdig et al., 1992; Moldovan, 2002).

\subsubsection{Intercellular Lumen}

In the second type, a lumen arises through a protrusion or migration of neighbouring ECs from newly formed or established vessels. Here, the lumen forms via the coordinated migration of several ECs that maintain their polarity and junctional contacts. Thus, an intercellular lumen is already formed at the start of bud formation and not, as usually postulated, after the formation of a solid endothelial cord. Initially, the breadth of the lumina is often only $0.1 \mu \mathrm{m}$ in size, and still attached to opposite cell membranes through point-like or cell-attached appositions and thus not yet traversable for cellular blood constituents. It has been shown that ECs having an intracellular lumen are frequently found at the tip of capillary sprouts with already existing channeled intercellular lumen and function practically as "connective pieces". The intracellular lumina can form contacts with the corresponding ECs of a neighbouring sprout and thus facilitate a continuity of the lumen through 
fusion of the vacuoles. Such tip-to-tip or tip-to-sprout fusions are known as anastomosis. This results in the fusing of the finger-like sprouts into a network of poorly perfused loops or arcades. In general, the fusion of sprouts exclusively with intracellular lumina over the entire length is not seen.

The results reported by Meyer et al. (1997) suggest that there are at least three different EC populations are involved in tube formation. One population, characterised by a phagocytic phenotype, migrates through the collagen gel creating channels through the matrix and thus creating a path for subsequent migration of a second population of ECs. The major morphological hallmark of the second migratory cell population is the presence of large numbers of intracellular vacuoles which coalesce and are extruded into the intercellular space. During their migration they envelope the initial phagocytic cells, which then die. It is suggested that the demise of the phagocytic cells is apoptotic in nature, although another possibility is that these cells are being "starved" of nutrients by the enveloping cells. A consequence of the secretion of vacuoles and programmed cell death is the extensive remodeling of the capillary tubes, resulting in expansion of the intercellular space into a lumen. Lumen extension is also mediated through addition of further EC into the sprout, which contributes to the multicellular nature of the lumen as well as the overall capillary size.

As noted earlier, integrins are known to link cell surfaces with the surrounding ECM and the underlying cytoskeleton (Schwartz \& Ingber, 1994; Hynes, 1992). They also regulate EC morphogenesis events, such as migration, sprouting, vacuole formation and lumen development (Davis \& Camarillo, 1996; Gamble et al., 1993). In a study by Bayless et al. (2000), anti- $\alpha_{2} \beta_{1}$ integrin antibodies completely blocked EC vacoule formation and completely interfered with subsequent lumen and capillary formation. This result is similar to that shown previoulsy by other investigators with multiple collagen-binding integrins, including $\alpha_{v} \beta_{3}$ and $\alpha_{5} \beta_{1}$ integrins (Gamble et al., 1993; Saelman et al., 1995). However, it is not entirley clear how integrins are involved in vacuole and lumen formation. Possibilities include: (i) activation of intracellullar signaling pathways that induce vacuole and lumen formation within the EC; and (ii) increasing cell-matrix affinity to initiate the pinocytic process. In this second case, clustering of $\alpha_{2} \beta_{1}$ on the plasma membrane could result in cell-matrix anchor points and vacuoles could develop through invagination of plasma membrane between such anchors. Furthermore, cell-cell adhesion sites may serve as anchors for the pinocytic process during intracellular lumen formation much like the cell-ECM contact sites for the intercellular structures. If one or both of the cells develop vacuoles at the cell-cell adhesion site, this would serve to create an ECM-free compartment and preliminary lumen between neighbouring cells (Davis \& Camarillo, 1996). Indeed, tight cell-cell contacts are clearly observed in the majority of capillaries in vivo (Cory et al., 1991), and blocking antibodies to these endothelial-junction-associated proteins have been shown to impair vacuole formation and fusion as well as reduce the frequency and mean length of the tube-like structures in a number of in vitro studies (see Yang et al. (1999) for a review).

\section{Maturation of the Neovasculature}

During tumor angiogenesis, the developing vessels are leaky and dependent on VEGF for survival (Benjamin et al., 1999). The end of this plasticity window is marked by the acquisition of a pericyte coating and the deposition of a basement membrane (Griffioen \& Molema, 2000). ECs recruit pericytes via the secretion of platelet-derived growth factor (PDGF), which acts as a mitogen and chemoattractant for pericyte precursors. On EC-pericyte contact, the pericytes exert an inhibitory effect on EC proliferation. Growth factors called angiopoietins and their receptors play a critical role in this process. These help stabilize mature vessels by promoting interaction between ECs and the newly recruited pericyte layer. In this section, we will first review the actions of the angiopoietins in angiogenesis control, and then discuss pericyte recruitment and function. We end the section by discussing how these processes link to various steps in the angiogenic cascade.

\subsection{Angiopoietins and Tie Receptors}

In addition to the VEGF-specific tyrosine kinase receptors (RTKs), another family of EC-specific RTKs called Tie receptors is involved in the control of blood vessel formation (Koblizek et al., 1998). The Tie family of receptors consists of two members, Tie-1 and Tie-2 (Tie-2 is also known as Tek). Tie-1 and Tie-2 are restricted mainly to the vascular endothelium and their precursors, but they are also expressed in some cells of hematopoietic linage (Kukk et al., 1997; Sato et al., 1998). Wong et al. (1997) found that Tie-2 was broadly expressed in the endothelium of the quiescent vasculature as well as in the endothelium undergoing angiogenesis, and the Tie- 2 receptor was activated in both quiescent and angiogenic tissues, suggesting that Tie-2 signaling may have a dual function in both vascular growth and vascular maintenance. The ligands for the Tie receptor have been identified as the angiopoietins, of which four are currently known (Ang-1 to Ang-4). The best characterized are Ang-1 and Ang-2, which bind with high affinity to Tie-2. However, none of the known angiopoietins bind to Tie-1, and it is unclear whether Tie-1 has independent ligands, or whether the angiopoietins can, under certain cirumstances, bind in some heteromerized complex to Tie-1 (Yancopoulos et al., 2000). The consensus at present is that Tie-1 function is related to EC differentiation and the establishment of blood vessel integrity (Griffioen \& Molema, 2000). 
Ang-1 induces a rapid activation (autophosphorylation) of Tie-2 in ECs (Maisonpierre et al., 1997), but depending on the cell examined, Ang-2 could either activate or antagonize Tie-2 (Yancopoulos et al., 2000). In a fibroblast cell line (NIH 3T3) Ang-2 was equivalent to Ang-1 in Tie-2 phosphoryolation (Maisonpierre et al., 1997). Furthermore, in hematopoietic BaF3 cells expressing Tie-2, both Ang-1 and Ang-2 activated the Tie-2 receptor, although the phosphorylation induced by Ang-1 in this case was more prominent (Sato et al., 1998). In vascular endothelial cells, however, Ang-2 binds but does not activate Tie-2. Instead, in this case, the natural role of Ang-2 may be to antagonize Ang-1 activation of Tie-2 (Lauren et al., 1998; Maisonpierre et al., 1997). For instance, Maisonpierre et al (1997) showed that a four- to eightfold excess of Ang-2 significantly inhibits Ang-1 activation in endothelial cell cultures. However the role of Ang-2 is far from clear, for in an ocular microvessel network Ang-2 can either stimulate angiogenesis or capillary regression, depending on whether or not VEGF is present (Lobov et al., 2002).

A direct comparison of the effects of an overexpression of VEGF and Ang-1 during angiogenesis was made by Thurston et al (1999) with transgenic mice overexpressing these factors in the skin. VEGF and Ang-1 play separate but crucial roles in angiogenesis: VEGF is believed to play an important part during the early stages of angiogenesis, whereas Ang- 1 acts during the subsequent remodeling and maturation of the vasculature. Thurston et al found that an overexpression of both of these factors resulted in an overall increase in vascularity in the ear skin of the K14-VEGF and K14-Ang1 mice. However, VEGF overexpressed blood vessels were small, tortuous and abnormally leaky. In contrast, vessels overexpressing Ang-1 were significantly larger than normal and were resistant to leaks caused by inflammatory agents. Interestingly, measurements showed that the increase in vessel area density with VEGF was mostly due to an increase in vessel length, whereas Ang-1 overexpression produced vessels with a much larger diameter. In combination, VEGF and Ang-1 gave rise to vessels that were thicker and longer than that of VEGF or Ang-1 overexpression alone. Thus, the effects of these two factors seem to be additive (Thurston et al., 1999).

Most research on the angiopoietins and Tie receptors has focused on developmental biology, with considerably less attention on the role of the angiopoietins in tumor-induced angiogenesis. An exception is that of Ahmad et al (2001), who examined the expression of Ang-1 and Ang-2 in human colon carcinoma specimens and cell lines, normal colon epithelium, and liver metastases. The current model is that Ang-1 is naturally expressed by cell types such as periendodothelial support cells (PCs) throughout the tissue at a constant background level and Ang-2 is expressed by ECs at areas of vascular remodeling and is absent from areas of quiescence or maturation (Tanaka et al., 1999). However, Ahmad et al. (2001) show that an alternative mechanism is that Ang-1 and Ang-2 are expressed at approximately equal levels in the normal quiescent tissue, whereas in the tumor epithelium the expression of Ang-2 exceeds that of Ang-1, leading to a net gain in Ang-2 function. They postulated that the net gain of Ang-2 activity may be an initiating factor for tumor angiogenesis. They also showed that the Ang-1 and Ang-2 protein was expressed by the transfected cell lines in addition to the PCs (Ang-1) and ECs (Ang-2).

In another study Koga et al (2001) investigate the expression of Ang-2 in glioblastoma tissue. Measurements were taken at three separate tumor regions: the area surrounding necrosis, an intermediate zone between the necrosis and the tumor periphery, and the tumor periphery. They found that the glioma cells themselves, and not only vascular ECs, expressed and secreted the Ang-2 protein, and that expression of Ang-2 was directly induced by hypoxia. The distribution of Ang-2 in the tumor was such that Ang-2 expression was high in the area surrounding necrosis and in the tumor periphery, and low in the intermediate region. A high expression of Ang-2, however, wasn't always associated with vessel growth. In the area surrounding necrosis, Ang-2 was high and the tumor vessels showed regression, whereas in the tumor periphery Ang-2 was also high but measurements indicated angiogenesis. The authors suggest that the contrary effects of Ang-2 may be due in part to the expression of Ang-1 and its receptor, Tie-2. That is, Ang-1 is downregulated by hypoxia (Enholm et al., 1997; Ristimaki et al., 1998), and would thus decrease around necrosis but would be maintained at normal levels in the tumor periphery. Tie-2 receptor expression has also shown to be increased by hypoxia (Willam et al., 2000), and hypoxia and VEGF are known to be inducers of Ang-2 expression in ECs (Koga et al., 2001). Thus the decrease of Ang-1 with increased Ang-2/Tie-2 expression may contribute to vessel regression in the regions surrounding necrosis. In contrast, Ang-1 expression is maintained and Ang-2 expression is increased in the periphery, and this may act to induce angiogenesis, rather than regression. For other tumor-related studies on angiopoietins see Lauren et al. (1998); Stratmann et al. (1998) and Peters et al. (1998).

Characteristics of blood vessels lacking angiopoietin-Tie-2 signaling components suggest that the regulation of PC and EC interaction may be an important function of the angiopoietins. Unlike vessels lacking VEGF or VEGF-receptor, vessels lacking Ang-1 or Tie-2 initially develop seemingly normal vasculature. However, this vasculature fails to mature and undergo normal remodeling, and it has been shown that these vessels are unable to associate properly with the underlying PCs (Asahara et al., 1998). PCs, such as smooth muscle cells and pericytes, have been shown to restrict the proliferation of vasculature ECs in a contact-dependent manner and maintain vessel integrity (details are given below) (Orlidge \& D'Amore, 1987). Tie-2 and Ang-1 knockout mice are embryonic lethal, and display defects in EC interactions with the basement membrane, and Ang-1 was found to regulate EC adhesion via integrins (Carlson et al., 2001). 
The initiation of vessel growth is associated with the loss of PCs from the existing vessels (Lauren et al., 1998). Conversely, the maturation of newly-formed vessels involves the recruitment and re-association of such PCs to the abluminal surface (Lauren et al., 1998). Therefore, Ang-1 doesn't appear to supply an instructive signal for angiogenic remodeling as such, but instead optimizes the way in which the vascular ECs integrate with their support cells and their environment (Yancopoulos et al., 2000). Conversely, the antagonizing effect of Ang-2 may cause vessel destabilization by inducing the weakening of EC interactions with PCs and the surrounding micro-environment (Koga et al., 2001), causing the ECs to revert to a more plastic state reminiscent of developing vessels (Yancopoulos et al., 2000). In this state the vessels are more sensitive to angiogenic factors and will either regress or grow depending on the availability of associated growth factors such as VEGF (Yancopoulos et al., 2000).

The pattern of angiopoietin-Tie-2 expression in tumors suggests the following model as one possible scenario (see Figure 5). First, the tumor is avascular. As the tumor grows, interior portions become hypoxic, resulting in marked induction of tumor-derived VEGF. Ang-2 expression is then increased in the vascular ECs either via the high levels of VEGF alone or in concert with other angiogenic factors such as bFGF (Lauren et al., 1998), or directly by hypoxia, which leads to the disruption of the interaction between nearby vascular endothelial cells and PCs as Ang-2 antagonizes the actions of Ang-1 by binding competitively to Tie2. This makes the ECs sensitive to VEGF signaling and thereby allows them to proliferate and migrate to form new vascular sprouts if VEGF is available. Ang-1 expression, whether tumor- or PC-derived, may then have a later role in the angiogenic cascade, involving vessel maturation of the newly formed vasculature through the re-association of ECs with supporting PCs. Note that Ang-2 mRNA in cultured ECs is downregulated in response to TGF- $\beta_{1}$, which is associated with the interaction of ECs with PCs (see below) (Mandriota \& Pepper, 1998). The lack of Ang-1 expression in high Ang-2/VEGF tumor regions such as those described by Koga et al (2001) (2001) may well explain the long-standing observations that some tumor vessels fail to mature, have poor associations with PCs, and are leaky (Yancopoulos et al., 2000).

\subsection{Pericytes and Platelet-Derived Growth Factor}

The term pericyte was initially used to describe cells that were found adjacent to capillaries (Zimmerman, 1923). These cell types have a small, oval cell body with multiple processes extending for some distance along the vessel axis. These primary processes then give rise to orthogonal secondary branches which encircle the vascular wall and penetrate the BM to directly contact the underlying endothelium (Thomas, 1999). At points of contact between ECs and pericytes, communicating gap junctions, tight junctions and adhesion plaques are present. This interaction is important for the maturation and remodeling of the vessels via the secretion of growth factors, such as TGF- $\beta_{1}$, or through modulation of the surrounding extracellular matrix (Allt \& Lawrenson, 2001). Further processes can be seen extending large distances between adjacent capillaries within the vascular network (Hirschi \& D'Amore, 1996). The number of pericytes varies considerably between different tissues and on vessels of different sizes. The proposed roles for pericytes include: regulation of capillary blood flow, as smooth muscle cell precursors, phagocytosis, and the control of capillary growth (for further details see the excellent review by Hirschi \& D’Amore (1996)). Hereafter we will focus on the regulatory role of pericytes in capillary growth.

Potential mechanisms by which vascular ECs recruit pericytes during angiogenesis include EC-derived soluble factors, changes in blood pressure and mechanical forces, and possibly cell communication by direct cell-cell contact (Hirschi \& D'Amore, 1996). It is well known that ECs and mural cells (the latter are smooth muscle cells or pericytes) can affect each other's behaviour via the paracrine exchange of extracellular diffusible factors, and in vitro studies suggest a role for the mitogenic and chemoattractant properties of the growth factors, PDGF, bFGF and VEGF (Yamagishi et al., 1999; Westermark et al., 1990). Evidence is particularly strong for the role of PDGF in angiogenesis. For example, Hirschi et al. (1999) have developed an in vitro co-culture system to study the relative contributions of cell-cell contact, autocrine and paracrine factors during vessel assembly. This demonstrates the directed migration of undifferentiated mesenchymal cells towards 
ECs in a PDGF-B dependent manner, as well as a significant increase in proliferation of these cells stimulated by PDGF-B. Furthermore, once the ECs and mesenchymal cells make contact, the mesenchymal cells are induced to differentiate toward a smooth muscle cell/pericyte fate. This cell-cell contact is also shown to inhibit the growth of both ECs and mural cells (Hirschi et al., 1999; Hirschi et al., 1997). The inhibition is not just a result of the heterotypic contact between these cells, however, since media collected from growth-inhibited cocultures also suppresses EC/mural cell proliferation, indicating that growth inhibition may be mediated by some soluble factor (Hirschi et al., 1999). A possible candidate for growth inhibition is TGF- $\beta_{1}$ (Hirschi \& D'Amore, 1996). Previous studies have shown that ECs and mural cells produce a latent form of TGF- $\beta$ when grown separately, and is activated in coculture (Hirschi et al., 1999). The ability of factors that interfere with the actions of plasmin to inhibit this activation points towards a role for plasmin in this activation process (Sato \& Rifkin, 1989). Futhermore, activated TGF- $\beta$ has also been shown to induce differentiation of mesenchymal cells towards a smooth muscle cell linage (Hirschi \& D’Amore, 1996; Hirschi et al., 1997).

A synthesis of the material discussed in this section is shown schematically in Figure 6, and can be summarized as follows. Alterations in the microenvironment, such as a disruption of the Ang-1/Ang-2 balance in favour of Ang-2, cause a loosening of the EC/periendodothelial cell contacts on the parent vessel, making ECs sensitive to angiogenic factors (VEGF, bFGF etc). These changes result in EC proliferation and migration, leading to new vessel growth directed towards the tumor. The immature vessels are initially devoid of a periendodothelial cell layer, making them leaky and dependent on VEGF for survival (Benjamin et al., 1999). This vulnerability is overcome through the acquisition of a smooth mural cell coating. ECs recruit undifferentiated mesenchymal cells via the synthesis and secretion of PDGF, which acts as a mitogen and chemoattractant for the mesenchymal cells, which migrate to the ECs where they make contact. The Ang-1/Ang-2 balance is readdressed in favour of Ang-1 somehow, either via the production of Ang-1 by the supporting cells themselves or a tumor production of this molecule (see section 5.1 for details), allowing the communication of vascular ECs with the newly recruited mesenchymal cells. The heterotypic interactions lead to the plasmin mediated activation of a latent form of TGF- $\beta_{1}$, which is produced by both ECs and mesenchymal cells. Activated TGF- $\beta$ inhibits EC and mesenchymal cell proliferation and induces changes in mesenchymal cells to express smooth muscle cell/pericyte makers. The acquisition of a pericyte coating, the deposition of a new BM by both cell types, and the cessation of vessel growth marks the end of the plasticity window and stabilizes the newly formed vasculature.

\section{The Role of Macrophages in An- giogenesis}

In addition to being able to recruit vascular ECs in a VEGFdirected fashion, tumor cells are also able to attract inflammatory cells, such as macrophages, which contribute to the production of angiogenic factors in an environment already enriched with angiogenic stimuli (Polverini, 1996b). Macrophages are scavenger cells derived from monocyte blood cells released from the bone marrow (Bingle et al.,

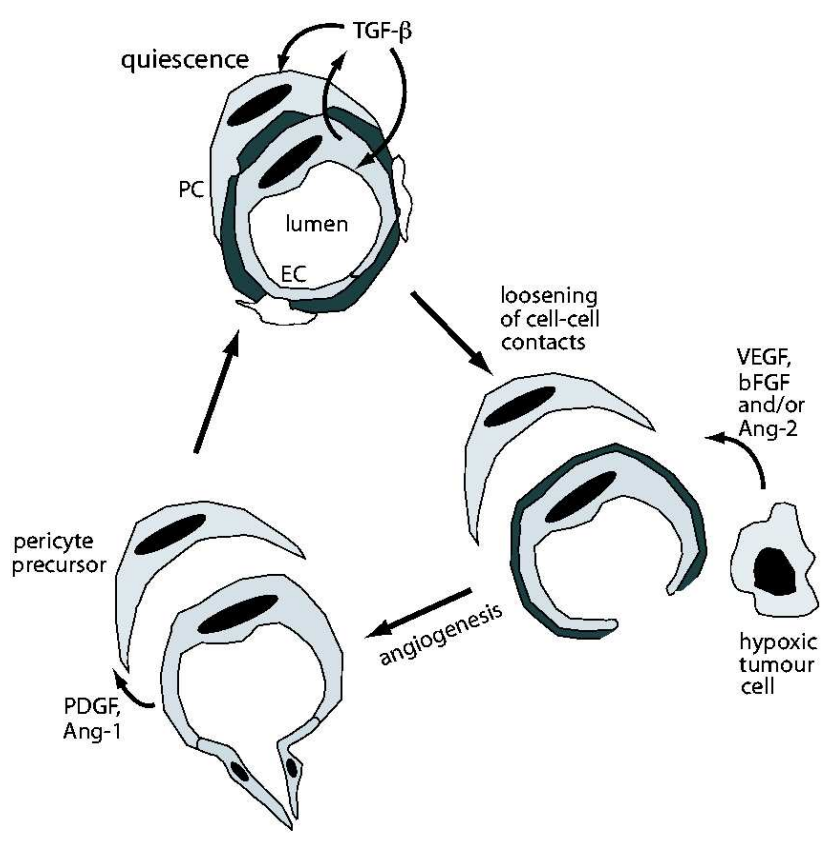

Figure 6: Pericyte/mural cell-endothelial cell interactions in angiogenesis. ECs activated by tumor-secreted angiogenic factors induce local PC-drop-off from the vessel, enabling them to overcome growth inhibition and start enzyme secretion, migration and proliferation. After EC activation and the formation of endothelial tube-like structures, ECs recruit mesenchymal cells via the synthesis and secretion of platelet-derived growth factor. The mural cell precursors then migrate to the EC where they make contact. On EC-mural cell contact, a latent form of TGF- $\beta$ produced by both cell types, is activated in a plasmin-mediated process. Activated TGF- $\beta$ induces the differentiation of mesenchymal cells into pericytes and smooth muscle cells, and inhibits EC proliferation, thereby contributing to the formation of a quiescent vessel. Subsequent microenvironmental alterations may tip the balance again in favour of EC growth stimulation and angiogenesis, leading to a repeat of the above process and further vessel growth and remodeling. Redrawn from Hirschi \& D'Amore (1996). 2002), and differentiate into resident macrophages once they cross the endothelium from the blood stream. Examples of resident macrophages include Kupffer cells in the liver, alveolar macrophages in the lung, and osteoclasts in the bone (Bingle et al., 2002). Macrophages are multifunctional cells with the potential to perform diverse functions, including the removal of debris, which they engulf by phagocytosis, antigen- 
presentation and cytotoxicity in immunity, tissue remodeling, inflammation (Polverini, 1996b; Bingle et al., 2002), and the production of a spectrum of soluble factors capable of directing endothelial cell growth and migration during tumor progression (Alberts et al., 2002; Polverini, 1996b). In this Section, we review the complex and multifaceted role of macrophages in tumor angiogenesis.

Malignant tumors often contain numerous macrophages, and these are referred to as tumor-associated macrophages (TAMs). Recent studies have shown that TAMs accumulate mostly in hypoxic areas in the tumor tissue (Leek et al., 1996), and a high density of macrophages in these areas has been correlated with tumor angiogenesis and reduced patient survival (Leek et al., 1996; Bingle et al., 2002). Tumor-produced chemotactic cytokines (chemokines) play an important role in monocyte recruitment into tumors (Bingle et al., 2002). Examples include monocyte chemotactic protein-1 (MCP-1) and macrophage colony stimulating factor (M-CSF) (Bingle et al., 2002). The most important of these is thought to be MCP-1, and other cell types, such as ECs, and fibroblasts have been shown to express this protein in human tumors (Mantovani, 1994). Furthermore, TAMs themselves have also been shown to express MCP-1 (Mantovani, 1994; Ueno et al., 2000). Indeed, up-regulation of MCP-1 expression by both tumor cells and TAMs has been shown to be positively associatied with high TAM accumulation in breast carcinoma (Wong et al., 1998).

MCP-1 was formerly considered to be specific for monocytes, but recently has been shown to be chemotactic for ECs as well (Salcedo et al., 2000). Moreover, in a recent study MCP-1 was shown to be a strong angiogenic factor, exerting a potency similar to that of VEGF-A (Goede et al., 1999). In addition to its pro-angiogenic activities, VEGF has also been shown to stimulate migration of macrophages via the VEGF receptor flt-1 (Bingle et al., 2002). In a study of breast carcinoma by Lewis et al. (2000), TAMs were found to accumulate in poorly-vascularized VEGF-positive areas of these tumors. Thus, in accordance with findings that VEGF is chemotactic for monocytes in vitro (Barleon et al., 1996), these findings indicate that VEGF may be chemotactic for macrophages in vivo, drawing TAMs up-gradient of VEGF into hypoxic tumor areas. This effect would be accentuated further via an autocrine loop of VEGF release from the TAMs themselves, since it is known that TAMs also respond to hypoxia by increasing their release of VEGF. These findings indicate that macrophages aid angiogenesis by accumulating in avascular tumor regions, where they co-operate with tumor cells to make further VEGF and initiate angiogenesis (Lewis et al., 2000). An alternative mode of attraction for macrophages into poorly vascularized, hypoxic tumor areas may be the movement of TAMs along an increasing gradient of necrotic cell debris, or simply that macrophages may migrate randomly around the tumor and become immobilized in the hypoxic region via a direct inhibition by hypoxia or a hypoxic inhibition of the macrophage chemokine MCP-1 (Bingle et al., 2002).

In addition to VEGF release, macrophages have been shown to respond to hypoxia by the up-regulation of a broad range of other soluble factors. In vitro studies have shown that macrophages are capable of producing in excess of 20 different types of molecules that can influence EC proliferation and migration during angiogenesis (Polverini, 1996b). Examples include TAM-derived cytokines IL- $1 \beta$ and TNF- $\alpha$, which have been shown to induce tumor expression of the potent proangiogenic protein angiogenin in vitro (Bingle et al., 2002; Etoh et al., 2000), and the monocyte-derived membrane-bound protein tissue factor (TF), which stimulates the migration of ECs during angiogenesis via the production of fibrin in the tumor (Zhang et al., 1994). Macrophages have also been shown to influence the composition of the ECM either by the production of ECM components or via the secretion of proteases capable of degrading the ECM (Polverini, 1996b). As noted earlier, ECM modification is a crucial part of the angiogenic process through its influence on EC shape and morphology, which in turn profoundly affects their behaviour. TAMs have been shown to be a major source of ECM degrading proteases including matrix metalloproteinases (MMPs), such as collagenase (MMP-1), gelatinase A and B (MMP-2 and MMP-9), stromelysin (MMP-3), and matrilysin (MMP-7) (Bingle et al., 2002). The serine protease uPA is synthesized by TAMs in a number of human tumors (Hildenbrand et al., 1999), and the lysosomal protease cathepsin D was shown to be expressed by TAMlike cells in invasive human bladder cancers (Lipponen, 1996). All of these proteases are able to promote angiogenesis by degrading the BM and disrupting the tissue architecture enabling new capillary growth.

Conversely, TAMs can also express anti-angiogenic functions. For instance, when appropriately activated, TAMs secrete not only pro-angiogenic factors but also synthesize the ECM molecule thrombospondin-1 (TSP-1), which is a potent inhibitor of angiogenesis (Polverini, 1996b; Moldovan, 2002) (angiogenesis inhibitors will be discussed in more detail in section 7). Moreover, besides scavenging apoptotic ECs and cellular debris, TAMs can also exert cytotoxicity towards the VEGFproducing tumor itself, but this requires activation by antibodies such as interferon gamma (IFN- $\gamma$ )(Bingle et al., 2002). Macrophage cytotoxicity can be either direct, such as the secretion of lytic factors directly into tumor cells (Bingle et al., 2002), or indirect via the production of TAM-derived cytokines such as IL-18 and IL-12, inducing anti-tumor effects in other cell types, including natural killer and cytotoxic T-lymphocytes (Trinchieri, 1998). Another indirect route by which TAMs disrupt angiogenesis is through the production of macrophage elastase (MMP-12). MMP-12 is a protease that on the one hand is able to promote angiogenesis and invasion through its proteolytic activity, but also releases potent angiogenesis inhibitors angiostatin from the cleavage of plasminogen and endostatin from collagen XIII (see section 7) (Bingle et al., 2002; 
Moldovan, 2002). Recently other MMPs matrilysin and gelatinase have also been shown to cleave plasminogen to generate angiostatin fragments (Patterson \& Sang, 1997).

An alternative model for angiogenesis is presented by Moldovan (2002). Their hypothesis is that TAMs go beyond the simple secretion of angiogenic factors and assist capillary formation by drilling a capillary path in the ECM as they penetrate the tumor as part of the inflammatory process. The vascular ECs then fill these empty spaces to form capillary sprouts as they grow outward from the parent vessel. This theory is based on facts such as under certain conditions ECs are unable to penetrate the ECM by themselves (Stetler-Stevenson, 1999), macrophages/monocytes are often co-localized in capillary sprouts (Moldovan, 2002), TAMs display receptors for angiogenic factors such as VEFG (Sawano et al., 2001), and macrophage chemotactic factor MCP-1 has also shown to be angiogenic (Goede et al., 1999). Furthermore, other studies show that even in pure in vitro EC cultures, capillary development involves at least three different EC subpopulations, with the subpopulation of ECs at the tip of the growing capillary displaying phagocytic characteristics (Meyer et al., 1997).

\section{Angiogenesis Inhibitors}

Since the early 70's when the importance of angiogenesis in tumor growth and metastasis was established, the discovery of the identity and biochemical mechanisms of substances that can inhibit angiogenesis has been a focal point in angiogenesis research. One can see from the sequence of events in angiogenesis described earlier that there are numerous points at which angiogenesis may in principle be blocked. The first inhibitors to be discovered were $\alpha$-interferon (Brouty-Boye \& Zetter, 1980) and PDGF (Taylor \& Folkman, 1982) which were shown to inhibit EC chemotaxis and proliferation, respectively. The discoveries of the angiogenesis inhibitors, angiostatin and endostatin (O'Reilly et al., 1994; O'Reilly, 1997; O'Reilly et al., 1997) have revolutionalized the field, and since then over 300 endogenous, natural or synthetic inhibitors have been described and more than 30 have entered clinical trials (Thompson et al., 2000). Understanding the mechanism by which various factors inhibit angiogenesis is critical to devising an effective therapeutic regimen. A tentative classification of these substances can be made on the basis of their inhibitory function, but the role of many newly-discovered inhibitors remains unknown. Similar as well as other classifications can be found in some excellent existing reviews due to Norby (1997); Tennant et al. (2000); Griffioen \& Molema (2000); Sephy (2000); Cao (2001) and Jimenez \& Volpert (2001). We discuss a few examples, but do not attempt to be complete.

\subsection{MMP inhibitors}

As we discussed in Section 3.2.2, the cleavage of the ECM is a crucial step in EC migration and is mediated by a group of proteins known as matrix metalloproteinases (MMPs). There are 26 known MMPs and their function is inhibited by a series of natural inhibitors called tissue inhibitors of metalloproteinases, or TIMPs. Four different TIMPs (TIMP-1,2,3,4) have been identified. TIMP-1 is produced by many cell types, including ECs, but its activity is often only induced at sites of inflammation. TIMP-2 is a protein isolated from a human melanoma cell line, and has been shown to be constitutivelyexpressed in various tissues. TIMP-3 exhibits a predominantly ECM-associated localization. TIMP-4 is a newly-described MMP inhibitor whose expression is tissue-specific. In general all TIMPS can inhibit all MMPs by binding noncovalently to either pro- or active-MMPs with a 1:1 stochiometry. Thus, TIMPs are involved in the control of pro-MMP activation as well as the inhibition of active proteinases. TIMPs do, however, show some differences in their abilities to form complexes with MMPs. For example, MT-MMP activity is hardly inhibited by TIMP-1, but very efficiently by TIMP-2 and TIMP-3 (Koolwijk et al., 1998). TIMP-1 forms a tight complex with pro-gelatinase B, whereas TIMP-2 instead forms a tight complex with pro-gelatinase A (Coussens \& Werb, 1996). The activities of the TIMPS themselves are controlled by diverse proteinases, including neutrophil elastase and stromelysin-3 (Coussens \& Werb, 1996). Barimastat and Marimastat are synthetic MMP inhibitors that inhibit MMPs by chelating zinc and preventing it from binding to the catalytic site (Tennant et al., 2000). Other synthetic inhibitors are AG3340, which inhibits MMPs 2,3,9,13, and 14, and Bay 12-9566, which is an inhibitor for MMPs -2 and -9 only. Despite significant side effects that have been revealed in phase 1 clinical trials, these MMP inhibitors have now entered phase 3 of clinical trials. Other phase 1 MMP inhibitors include Neovastat, Col-3, and BMS-275291. Finally, carboxyamidotriazole (CAI) decreases the production of MMPs and therefore, inhibits EC migration (Griffioen \& Molema, 2000).

\subsection{EC Toxins}

Angiogenesis can also be inhibited by direct inhibition of EC activity. Thalidomide was recently shown to possess antiangiogenic activity in a corneal micropocket assay (D'Amato et al., 1994), and is considered to be very toxic. Another EC toxin is fumagillin, which was discovered in 1990 (Ingber et al., 1990). Since then, many synthetic analogues have been discovered, the best-known of which is TNP-470. Other clinically-tested EC toxins include combrestatin A-4, which is a natural product derivative of the African tree, and compounds Comberatum caffrum and squalamine, which are derivatives 
of dogfish shark extract. Combrestatin A-4 inhibits tubulin polymerization, while squalamine inhibits the sodium-hydrogen exchanger NHE3 (see Tennant et al. (2000) and references therein).

One of the first angiogenesis inhibitors that was found to be produced by fibroblast cells is Thrombospondin-1 (TSP-1) (Rastinejad et al., 1989; Good et al., 1990). TSP-1 is a $450 \mathrm{KDa}$ glycoprotein secreted by many normal and tumor cells, but secretion by tumor cells is significantly lower. TSP-1 secretion is upregulated by the p53 tumor suppressor protein in fibroblasts (Dameron et al., 1994) and mammary epithelial cells (Volpert et al., 1995). It is also secreted by ECs (Frazier, 1987; Wu et al., 2001), macrophages (Hirschi \& D'Amore, 1996), and pericytes (Wu et al., 2001). TSP-1 along with the other 4 members of its family (TSP-2 to TSP-5) mediate interactions between extracellular matrix molecules such as collagen and cellular integrin receptors such as $\alpha_{\nu} \beta_{3}$ (Detmar, 2000; Bornstein, 1995). Jimenez \& Volpert (2001) provide evidence that TSP-1 induces CD36 receptor-mediated apoptosis of capillary EC, although some of the details of the proposed mechanism, which involves the activation of caspase proteases, still remain unknown.

\subsection{Angiogenesis Activator Blockers}

Several substances that inhibit angiogenesis by interfering with tumor angiogenic factors (VEGF, bFGF, PDGF) have been identified. SU5416 has been shown to inhibit the VEGFR-2 pathway by inhibiting tyrosine kinase catalysis, which leads to inhibition of EC proliferation (Fong et al., 1999). PTK787/ZK 22584 is another VEGF receptor blocker, while SU6668 was shown to inhibit PDGF, FGF receptors as well as VEGFR-2 receptor (Tennant et al., 2000). One of the first inhibitors to be discovered was interferon- $\alpha$ (IFN- $\alpha$ ) (Brouty-Boye \& Zetter, 1980). It was found that it inhibits EC migration and proliferation by blocking VEGF and bFGF expression (Inoue et al., 2000). In addition to IFN- $\alpha$, other immuno-cytokines have recently been shown to inhibit angiogenesis both in vitro and in vivo. They include platelet factor-4 (PF-4) (Maione et al., 1990), chemokine gro-beta (Gro- $\beta$ ) (Cao et al., 1995), Interferon- $\gamma$ (IFN- $\gamma$ ) (Voest et al., 1995), interferon- $\gamma$-inducible protein-10 (IP-10) (Angiolillo et al., 1995), interleukin-12 (IL-12) (Voest et al., 1995), and interleukin-18 (IL-18) (Cao et al., 1995). Thus, there is some evidence of tight interaction between the immune and vascular systems (Cao et al., 1995).

\subsection{Integrin Binding Blockers}

We discussed in Section 4 how integrins mediate cell-ECM interactions. In particular, the $\alpha_{\nu} \beta_{3}$ integrin is a receptor protein required for the survival and maturation of blood vessels (Brooks et al., 1994a; Brooks et al., 1994b). It mediates cellular adhesion to vitronectin, fibronectin, fibrinogen, laminin, collagen, von Willebrand factor, osteopontin and thrombospondin (Varner et al., 1995; Polverini, 1996a). Vitaxin is a humanized monoclonal antibody that inhibits the $\alpha_{\nu} \beta_{3}$ integrin and results in a reduction of TGF- $\beta$ expression, an increase in EC apoptosis and causes shrinkage of vessels in the rabbit cornea (Coleman et al., 1999). The drug was well tolerated and the only side effect observed in phase 1 clinical trials was fever. A second antagonist of $\alpha_{\nu} \beta_{3}$ integrin is EMD 121974, which prevents ligation of the $\alpha_{\nu} \beta_{3}$ integrin, which, in turn, results in apoptosis of EC proliferating cells (Brooks et al., 1994a).

\subsection{Inhibitors as Fragments of Large Precursor Molecules}

An important step in angiogenesis research was the discovery of inhibitory activity in fragments of large proteins, which have weak or non-existent inhibitory activity themselves. Homandberg et al. (1985) first showed that a fragment of fibronectin inhibited EC proliferation in vitro, while the intact fibronectin molecule was not an inhibitor itself. A similar obsevation was made for a fragment of prolactin (Ferrara \& Davis-Smyth, 1997; Clapp et al., 1993). Folkman and co-workers later developed a strategy to discover circulating angiogenesis inhibitors generated by human tumors (Chen et al., 1995; Hanahan \& Folkman, 1996), which led to the isolation of angiostatin as a fragment of plasminogen (O'Reilly et al., 1994), endostatin as a fragment of collagen XVIII (O'Reilly et al., 1997) and serpin antithrombin as a fragment of antithrombin (O'Reilly et al., 1999). Gupta et al. (1995) (Gupta et al., 1995) have discovered that truncaten, a fragment of PF-4, has 50 times higher angiogenic activity than PF-4, whereas a fragment of matrix metalloproteinase-2 (MMP-2) termed PEX, was found to have increased antiangiogenic activity (Brooks et al., 1998). Vasostatin was isolated as a fragment of calreticulin (Pike et al., 1998), whereas restin (Ramchandran et al., 1999), arresten (Colorado et al., 2000), canstatin (Kamphaus et al., 2000), and tumstatin (Maeshima et al., 2000) are also recently discovered potent angiogenic inhibitors isolated as fragments of collagen XV, IV $\alpha 1$, IV $\alpha 3$, and IV $\alpha 3$, respectively. The importance of the discovery of this "hidden" inhibitory activity is best illustrated by the findings of O'Reilly et al. (1997) (O'Reilly et al., 1997) for endostatin, perhaps the most potent inhibitor discovered to date. They showed that endostatin can cause regression of primary tumors to a dormant state, decrease metastatic growth to extremely low levels, is non-toxic and is effective in many tumor types. Bohem et al. (1997) have further shown that, as opposed to many other chemotherapeutic drugs, endostatin does not exhibit any drug resistance in mice bearing Lewis lung carcinoma, T241 fibrosarcoma or B16710 melanoma. 
The elucidation of angiostatin's and endostatin's inhibitory activity has been avidly pursued following their discovery. Dhanapal et al. (1999) suggested that endostatin affects EC survival via the induction of an imbalance between the antiapoptotic proteins Bcl-2 and Bcl- $\mathrm{X}_{L}$, and the pro-apoptotic protein Bax. Claesson-Wells et al. (1998) have shown that angiostatin induces EC apoptosis independently of the integrin-binding motif RGD, while Yamagushi et al. (1999) demonstrated that endostatin inhibits VEGF-induced migration and tumor growth independently of zinc binding. It has been hypothesized that EC proliferation is mediated by intracellular transient elevations in $\mathrm{Ca}^{2+}$ concentration, and Jiang et al. (2001) have shown that angiostatin and endostatin affect these transient $\mathrm{Ca}^{2+}$ elevations through a PLC coupled pathway, which involves intracellular $\mathrm{IP}_{3}$ production. Moreover, Karihaloo et al. (2001) pointed out that endostatin significantly reduces branching morphogenesis in renal epithelial cells and ureteric bud. Bloch et al. (2000) have shown that endostatin has a significant influence on blood vessel maturation in wound healing. Furthermore, Stack et al. (1999) also showed that t-PA has a binding site for angiostatin as well as for its substrate plasminogen, which when occupied prevents the t-PA matrix-mediated activation of plasminogen to plasmin, thereby resulting in a reduced invasive activity. Despite these and other findings, the biochemical mechanisms by which angiostatin and endostatin inhibit EC migration and proliferation remain unclear, but significant progress has been made toward understanding the origin and effects in more molecular detail Han \& Liu (1999); Bloch et al. (2000); Kim et al. (2000); Ergun et al. (2001); Hanai et al. (2002); Karihaloo et al. (2001); Shichiri \& Hirata (2001); Rehn et al. (2001); Lee et al. (2002); Furumatsu et al. (2002).

\section{Mathematical Modeling}

Mathematical models of tumor-induced angiogenesis fall into three major categories: (i) continuum models that treat the EC density and chemical species as continuous variables that evolve according to a reaction-diffusion system, (ii) mechanochemical models that incorporate some of the mechanical effects of EC-ECM interaction on cell motion, in addition to the diffusible chemical species, (iii) discrete, cell-based models in which cells are treated as units and move, grow and divide according to prescribed rules. Because of the complexity of the entire process, the models to date treat only a subset of the factors involved in angiogenesis.

\subsection{Continuum models}

A typical continuum model consists of a number balance on the ECs, which is coupled nonlinearly with the set of mass balances describing selected factors in the EC environment. Early modeling efforts concentrated only on the chemotactic response of ECs to tumor angiogenic factors, while later models also considered the haptotactic response of ECs to adhesive gradients, usually of fibronectin, as well as the role of the inhibitors in the angiogenic process.

A typical continuum model of the type described in detail later can be formulated as follows. Let $\Omega$ be a domain in $R^{n}$, $\mathrm{n}=1,2$ or 3 , let $x \in R^{n}$ be the spatial coordinate, and let $t$ denote the time; then the governing equations are

$$
\begin{aligned}
& \frac{\partial n}{\partial t}=-\nabla \cdot J+f(n, c) \\
& \frac{\partial c}{\partial t}=D \nabla^{2} c+g(n, c) .
\end{aligned}
$$

Here $n(x, t)$ is a vector comprised of the densities of all cell types involved in the process (e.g., ECs, pericytes, etc.), $c(x, t)$ is a vector containing the concentrations of substances influencing migration and/or proliferation, such as TAF, protease enzymes, fibronectin, and angiogenic inhibitors. $J$ is the flux of endothelial cells, which comprises a random diffusive component, as well as a set of tactic (directed) components, and can be written in the form

$$
J=-D_{n} \nabla n+\sum_{i} \chi_{i}(c) n \nabla c_{i}
$$

The chemicals that are responsible for these tactic components of the flux could be TAF (chemotaxis), fibronectin (haptotaxis), etc. $\chi_{i}(c)$ is the tactic sensitivity of ECs in response to a gradient of the $i^{t h}$ tactic component. Usually it is assumed that $\chi_{i}$ depends only on the concentration of the corresponding tactic chemical, but in principle, it may depend on all of them, as has been analyzed in another context (Painter et al., 2000). The function $f(n, c)$ models the combined effects of cell proliferation and apoptosis, while $g(n, c)$ is a vector function describing the dynamics of production, uptake, and degradation of the chemical components included in the model. Finally, $D$ is a diagonal matrix containing the diffusion coefficients of each chemical species.

The simplest type of boundary conditions are no-flux conditions for the EC density and Dirichlet boundary conditions for the chemicals. The latter may or may not depend on time. Thus

$$
J=0 \quad \text { and } \quad c=c_{0}(t) \quad \text { on } \quad \partial \Omega
$$


Continuum models have been developed to describe both the onset of angiogenesis and the later stages of the angiogenic process, and as we will see later, the boundary conditions may be more complex. We begin with models for the onset of angiogenesis and then discuss models for the dynamics of the angiogenic process from initiation until the tumor is reached.

\subsubsection{The Initiation of Angiogenesis}

One of the first steps in angiogenesis is the binding of VEGF produced in a tumor by receptors on a nearby primary vessel. As is detailed in Section 3.1.3, this initiates a cascade of processes that includes loosening of contacts between ECs in the primary vessel, degradation of the basement membrane, leakage or active transport of plasma proteins into the surrounding tissue, movement of ECs or their precursors into the tissue, and ultimately the initiation of a capillary bud. Thus a first issue for mathematical modeling is to understand the first stage of angiogenesis, which determines the localization along a primary vessel of the site at which a capillary bud forms. Does the site simply coincide with the site at which the VEGF concentration is highest, or is a more complex process involved?

The first model that addresses the question of site location is due to Orme \& Chaplain (1996). This model is based on reaction-diffusion and haptotaxis mechanisms and invokes equations of the type given by (1) on a one-dimensional domain, thought of as coincident with the primary vessel wall. Bud initiation is described as a local accumulation of cells at a particular site, and in the model this results from a diffusion-driven instability of the type first described by Turing (1952). In effect this model predicts that the site of bud initiation is not related to the local distribution of VEGF in the tissue, but rather, that it is determined by an intrinsic wavelength set by the interaction of reaction, diffusion and taxis. Levine, Sleeman and Hamilton continued this line of investigation but introduced more complex models that involve other processes as well. In Levine et al. (2001b) the site is determined from the interaction of ECs, TAF, proteases, and fibronectin, while in Levine et al. (2000) the dynamics of angiostatin, pericytes, and macrophages are included. In addition, a second type of TAF secreted only by the macrophages is introduced. We shall focus on the second model, hereafter referred to as LSN-II, because it incorporates and supersedes the first model.

LSN-II incorporates a role for pericytes and macrophages as well as that for ECs, and postulated mechanisms for the function of angiostatin. Two possible mechanisms for the role of angiostatin in endothelial and pericyte cell migration are considered. In the first, angiostatin acts directly as an inhibitor of a protease, while in the second, it functions indirectly by playing the role of a substrate for the production of a protease inhibitor. Both mechanisms result in a decrease in the rate of fibronectin degradation. This in turn inhibits endothelial and pericyte cell migration, as ECs are assumed to move towards lower concentrations of fibronectin and high concentrations of uninhibited protease, while pericyte migration is only facilitated in regions of low fibronectin concentration. Macrophage migration is assumed to be independent of protease and fibronectin concentrations, but depends only on the concentration of angiogenic factors secreted by the tumor cells. In addition, the model considers two types of angiogenic factors, one that is secreted by tumor cells and binds to receptors on the surface of macrophages, and a second that is secreted by macrophages and binds to receptors on the surface of ECs. Notably, neither of the two types of angiogenic factors is considered to bind to receptors on the surface of pericytes.

The equations in LSN-II reflect mass balances for the TAF secreted by tumor cells, the proteases and fibronectin, the angiogenic factors secreted by macrophages, angiostatin, and the inhibitor produced by angiostatin. These mass balances are all ordinary differential equations, because diffusion of these species along the parent vessel is assumed negligible compared to reaction. These equations are in turn coupled with partial differential equations that govern the number densities of ECs, pericytes and macrophages along the length of the parent blood vessel. The derivation of the latter equations is based on the continuum theory of reinforced random walks developed in Othmer \& Stevens (1997). The nonlinear coupling of the partial differential equations with the set of ordinary differential equations arises from the nonlinear dependence of the transition probabilities on the concentration of protease enzyme, fibronectin and TAF.

The notation for the dependent variables is as follows. All concentrations are in micromoles/liter.

$$
\begin{aligned}
& n=\text { endothelial cell (EC) density } \\
& \tau_{1}, \tau_{2}, \tau_{3}=\text { transition probability for ECs, pericytes, and macrophages, respectively. } \\
& \sigma=\text { pericyte cell (PC) density } \\
& m=\text { macrophage cell density } \\
& u=\text { concentration of the angiogenic factors secreted by tumor cells } \\
& v=\text { concentration of angiogenic factors secreted by macrophages } \\
& c=\text { concentration of proteolytic enzymes }
\end{aligned}
$$


$f=$ density of capillary wall represented by fibronectin

$a=$ concentration of angiostatin

$i_{a}=$ protease inhibitor density

$c_{a}=$ active proteolytic enzyme density.

Further, define the functions

$$
\begin{aligned}
\tau_{1}\left(c_{a}, f\right) & =\left(\frac{c_{a}+a_{1}}{c_{a}+a_{2}}\right)^{\gamma_{1}}\left(\frac{f+\beta_{1}}{f+\beta_{2}}\right)^{\gamma_{2}} \\
\tau_{2}(f) & =\left(\frac{f+a_{3}}{f+a_{4}}\right)^{\gamma_{3}} \\
\tau_{3}(u) & =\left(\frac{u+\beta_{3}}{u+\beta_{4}}\right)^{\gamma_{4}} \\
c_{a} & =\frac{c}{1+\nu_{e} i_{a}} .
\end{aligned}
$$

Then the governing equations for LSN-II are as follows.

$$
\begin{aligned}
\frac{\partial n}{\partial t} & =D_{1} \frac{\partial}{\partial x}\left(n \frac{\partial}{\partial x}\left(\ln \frac{n}{\tau_{1}}\right)\right) \\
\frac{\partial \sigma}{\partial t} & =D_{2} \frac{\partial}{\partial x}\left(\sigma \frac{\partial}{\partial x}\left(\ln \frac{\sigma}{\tau_{2}}\right)\right) \\
\frac{\partial m}{\partial t} & =D_{3} \frac{\partial}{\partial x}\left(m \frac{\partial}{\partial x}\left(\ln \frac{m}{\tau_{3}}\right)\right) \\
\frac{\partial u}{\partial t} & =-\frac{\lambda_{2} u m}{1+\nu_{2} u}+u_{r}(x, t) \\
\frac{\partial v}{\partial t} & =\frac{\lambda_{2} u m}{1+\nu_{2} u}-\frac{\lambda_{1} v n}{1+\nu_{1} v}+v_{r}(x, t) \\
\frac{\partial c}{\partial t} & =\frac{\lambda_{1} v n}{1+\nu_{1} v} \\
\frac{\partial f}{\partial t} & =\beta\left(f_{M}-f\right) f n-\frac{\lambda_{4} c_{a} f}{1+\nu_{4} f} \\
\frac{\partial a}{\partial t} & =-\frac{\lambda_{3} a n}{1+\nu_{3} a}+a_{r}(x, t) \\
\frac{\partial i_{a}}{\partial t} & =\frac{\lambda_{3} a n}{1+\nu_{3} a}
\end{aligned}
$$

The equations (5)-(17) contain 31 parameters that are estimated from a variety of sources: the $a_{i}, \beta_{i}, \gamma_{i}, i=1, \cdots, 4$ are empirical constants; the $D_{i}, i=1,2,3$ are the diffusion coefficients of ECs, PC and macrophages, respectively; the $\lambda_{i}, \nu_{i}, i=1, \cdots, 4$ and $\nu_{e}$ are reciprocal Michaelis-Menten constants for the secretion of proteolytic enzymes $(i=1)$ from ECs, secretion of TAF from macrophages $(i=2)$, secretion of protease inhibitor from ECs $(i=3)$, and consumption of fibronectin through its interaction with active proteolytic enzymes. In addition, $\beta$ is the rate constant for the production of fibronectin from ECs $f_{M}$ is the density of fibronectin in the capillary wall, $a_{r}(x, t)$ is the rate of supply of angiostatin, which is assumed to be constant, and $u_{r}(x, t)$ and $v_{r}(x, t)$ are the rates of supply of TAF due to the tumor and macrophages, respectively. The tumor supply of TAF is given by

$$
u_{r}(x, t)=u_{0} k_{m}[(1-\cos (2 \pi x))]^{m} e^{-\theta t},
$$


where $u_{0}, K_{m}, m$ and $\theta$ are model parameter values. Numerical values of all parameters can be found in LSN-II.

Zero-flux boundary conditions are imposed on equations (9), (10) and (11) :

$$
n \frac{\partial}{\partial x}\left(\ln \frac{n}{\tau_{1}}\right)=\sigma \frac{\partial}{\partial x}\left(\ln \frac{\sigma}{\tau_{2}}\right)=m \frac{\partial}{\partial x}\left(\ln \frac{m}{\tau_{3}}\right)=0
$$

and the initial conditions for the system (9)-(17) are:

$$
\begin{aligned}
& n(x, 0)=n_{e}, \sigma(x, 0)=\sigma_{e}, m(x, 0)=m_{c} \\
& u(x, 0)=0, v(x, 0)=0, c(x, 0)=0 \\
& a(x, 0)=0, i_{a}(x, 0)=0, f(x, 0)=f_{M}
\end{aligned}
$$

where $n_{e}, \sigma_{e}$, and $m_{e}$ are constant cell densities.

The authors use the general model to numerically study the effect of the two mechanisms of angiostatin function on the dynamics of endothelial, pericyte and macrophage cell density. When angiostatin is not included in the model, one peak is predicted for the endothelial and macrophage cell densities (Figure 7(a) and (b)) and two are predicted for the pericyte cell density (Figure 7(c)) for the parameters used in LSN-II.
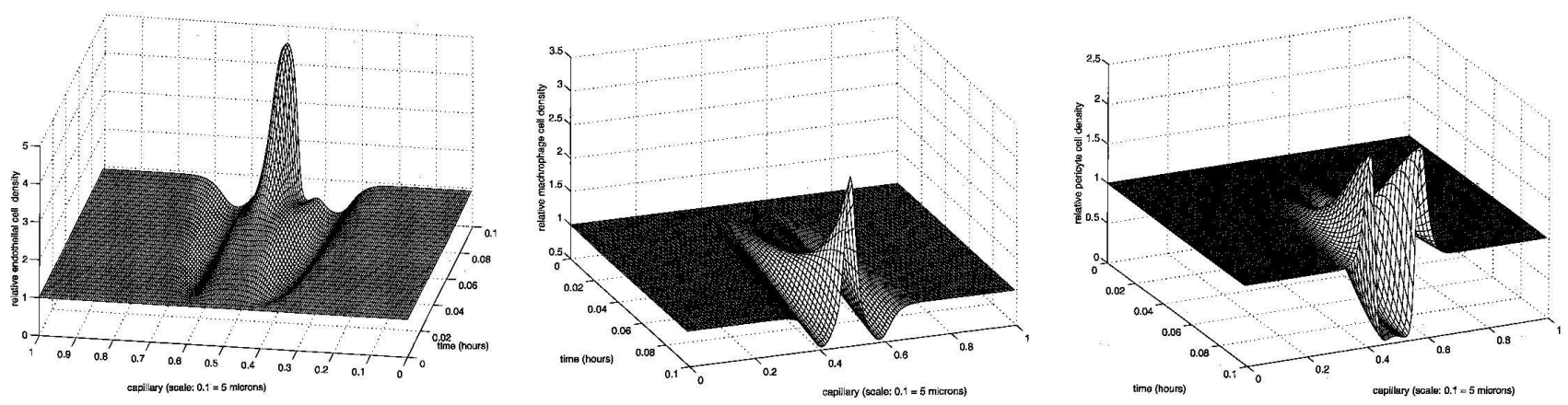

Figure 7: The time course of the endothelial (left), macrophage (center), pericyte (right) densities predicted from LSN-II in the absence of angiostatin. In the simpler model wherein pericytes, macrophages and angiostatin are not included, the EC density exhibits two peaks (results not shown; see figure 5.2 in Levine et al. (2001b).

When angiostatin is supposed to function indirectly by producing a protease inhibitor, it significantly reduces the peak of the EC density (Figure 8 (left)), causes both pericyte density peaks to completely disappear (Figure 8 (middle)), and leaves the macrophage cell density essentially unaffected (Figure 8 (right)). On the other hand, when angiostatin functions directly as the protease inhibitor, the inhibition effects are less pronounced. In either case the peak in the EC density is located at the peak of the initial TAF distribution, and localization of ECs there results form the assumption that the transition probability $\tau_{1}$ depends on the concentration of both active protease and fibronectin (cf., 5), and both factors are produced in proportion to the EC density. Thus there is a strong positive feedback, and this leads to the sharp localization of ECs. In fact there is a finite time at which the solution blows up, as is the case with a variety of reinforced random walk models (Othmer \& Stevens, 1997).

LSN-II represents an ambitious attempt to theoretically predict the site of sprout formation by incorporating detailed mechanisms for both cell movement and the biochemical steps involved. The model can predict some of the qualitative features of the observations, but much more experimental work is needed to determine whether the postulated mechanisms have a sound basis. Even at the acknowledged level of uncertainty about the biochemistry involved, there are aspects of the LSN-II model which require further study, and we point these out to serve as a focus of future work.

(i) The authors assume that tumor-produced TAF only affects the macrophages, not the ECs, and the latter in turn produce a different TAF. As a result, protease secretion is only stimulated by the angiogenic factors secreted by macrophages, and not by the factors secreted by the tumor cells ( $c f(14)$ ). In addition, it is assumed that TAF signal transduction occurs only via receptor internalization, but the growth factor receptors are tyrosine kinases and the primary step is phosphorylation of intracellular components. Thus one expects that the production of $v(c f(13))$ is proportional to the fraction of receptors occupied, and that $u$ is not degraded in this step, whereas the model invokes a Michaelis-Menten step for the transduction. 

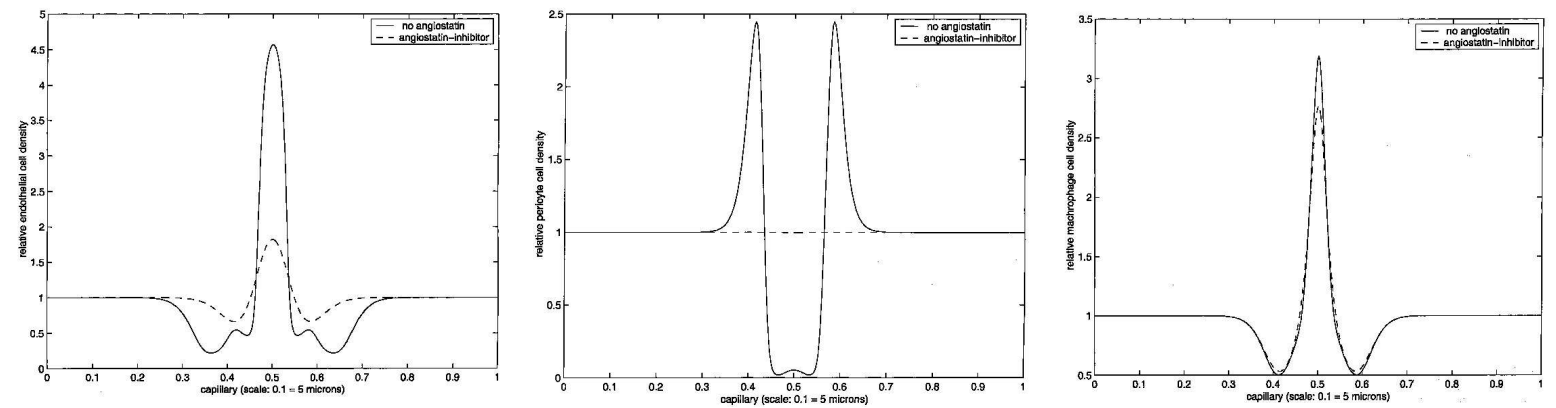

Figure 8: The role of angiostatin in LSN-II. EC (left), pericyte (middle) and macrophage (right) densities at $\mathrm{t}=0.1 \mathrm{hr}$ when angiostatin is included (dashed line) and absent (solid line).

Internalization is known to occur in some systems as a means of desensitization, but is usually slower than the primary transduction step.

(ii) The degradation of some key components is not included, even though some are known to have relatively short half-lives. These include the protease ( $c f(14))$, the angiogenic factors ( $c f(12)$ and (13)) and angiostatin ( $c f(16))$. While the lack of the degradation term in the protease enzyme equation is justified by a "worst case scenario" argument, there is no justification for the case of the angiogenic factor and angiostatin equations. The omission of the degradation of the angiogenic factors maybe particularly important, since it is known that VEGF, for example, has a half-life of the order of 3 minutes (Folkman, 1995a). Turnover is incorporated in a different model analyzed in Levine et al. (2001a), which is discussed later.

(iii) The predicted profiles of the EC density and other components is determined by the functional form of the dependence of the transition probabilities on the proteolytic factors, fibronectin, and the angiogenic factors ( $c f$. (5)-(7)), but at this stage one cannot even determine whether the model of a reinforced random walk is appropriate, much less determine the functional forms. This is a step for which much more experimental and theoretical work is needed.

(iv) Both models ignore diffusion of TAF, angiostatin and fibronectin (12), (13), and (15). The authors use two arguments to justify this assumption: (i) they assert that diffusion of these species takes place on a much slower time scale than reaction, and (ii), they observed that the incorporation of diffusion in the model increases the width of the density peaks unrealistically. However, if TAF diffusion is too slow to be included in the fast dynamics of the model, that rationale would also apply to the much slower diffusion of ECs included in the model? Perhaps the widening of the peaks with the inclusion of diffusion is indicative of the need for another change in the model and not the omission of TAF diffusion.

(v) The introduction of a second type of angiogenic factor complicates the model unnecessarily as (i) it requires additional model parameter values, (ii) it leads to additional assumptions such as preferential binding of one type of TAF to a certain cell type and not to the other. Given the present state of knowledge, it would be simpler and appropriate to ignore the role of the macrophages, as is done in more recent work Levine et al. (2001a).

(vi) Throughout the system (9)-(17) the Michaelis-Menten reduction is invoked to eliminate intermediate species, but this does not lead to the rational functions used in the kinetic terms when the enzymes or receptors involved are not conserved.

In summary, a number of important insights are gained from this model, but so much is unknown about the detailed biochemistry that predictions from any model are very qualitative at this stage. Despite this, these papers represent an important first step toward understanding the onset of angiogenesis using biochemically-based rather than phenomenological arguments. Future work is needed to systematically explore the effect of changes in the postulated mechanisms, and some of this is done in the recent paper by Levine et al. (2002).

\subsubsection{The Dynamics of Angiogenesis}

The first model that was developed to describe the dynamics of tissue formation and angiogenesis is that of Zawicki et al. (1981). The model comprises three reaction-diffusion equations for the density of red blood cells, tissue cells, and vessel cells, but since the identity of growth factors that stimulate EC migration and proliferation was unknown at the time, the model did not include any interaction between the three cell populations and any growth factors, nor did it incorporate chemotaxis. Since then, a variety of continuum models have been suggested to describe the dynamics of angiogenesis.

A novel approach to the growth of sprouts in the cornea was introduced by Balding \& McElwain (1985), who developed separate equations for two distinct components of the EC density, the sprout proper and the tip. This idea is based on the 
assumption that the tip cells serve to guide the EC, which form the sprouts immediately behind the tip. This approach has several advantages: (i) it can account for the experimental observation that EC proliferation occurs mainly near the tip of the vasculature and is rather infrequent in the main body of the growing vasculature, and (ii) it can account for branching and anastomosis. Byrne \& Chaplain (1995) \& (1996) have pursued this approach with a one-dimensional model describing the dynamics of TAF concentration, EC density at the tip of the vasculature, and EC density in the sprout. In this model the tumor is at $x=0$ and the parent vessel is at $x=1$. The governing equations for the tip density $n(x, t)$, measured in units of tips per unit area, the vessel density $\rho(x, t)$, measured in units of length per unit area, and the TAF concentration $c(x, t)$ are as follows.

$$
\begin{aligned}
\frac{\partial n}{\partial t} & =\frac{\partial}{\partial x}\left[D \frac{\partial n}{\partial x}-n \chi \frac{\partial c}{\partial x}\right]+a_{0} \rho c+a_{1} H(c-\vartheta) n c-\beta n \rho \\
\frac{\partial \rho}{\partial t} & =D \frac{\partial n}{\partial x}-n \chi \frac{\partial c}{\partial x}-\gamma \rho \\
\frac{\partial c}{\partial t} & =\frac{\partial^{2} c}{\partial x^{2}}-\lambda c-a_{1} H(c-\vartheta) n c
\end{aligned}
$$

These are subject to the boundary conditions

$$
\begin{aligned}
\rho(1, t) & =\rho_{\min }+\left(1-\rho_{\min }\right) e^{-k t} \\
n(0, t) & =0 \\
n(1, t) & =n_{L} e^{-k t} \\
c(0, t) & =1 \\
c(1, t) & =0
\end{aligned}
$$

and the initial conditions

$$
\begin{aligned}
\rho(x, 0) & =\delta(1) \\
n(x, 0) & =n_{L} \delta(1) \\
c(x, 0) & =\delta(0) .
\end{aligned}
$$

The second term on the right-hand side of (20) represents branching, the third describes proliferation, and the fourth describes the loss of tip cells to vessel anastomosis. Proliferation of the tip cells is supposed to stop when the TAF concentration is below the threshold $\vartheta$. Note however that there is no conversion of tip cells to vessel cells; the vessel density changes only due to the motion of the tip and to decay; there is no loss term from branching and no gain term from anastomosis or growth in (21). In effect it is supposed that cells are added to a growing vessel only at the parent vessel at a rate which is specified at the boundary, and cells move outward at a rate dictated by the movement of the tip. Thus vessel density will decrease if the tip speeds up. The equation for the concentration of TAF includes diffusion, linear decay and loss leading to growth of ECs. It is somewhat unrealistic to assume that the consumption of a unit of TAF produces precisely the same change in the tip density; more generally another proportionality coefficient expressing the yield per unit of TAF would be expected in the first equation.

To analyze this model computationally we used a finite-element-based discretization in space and a $4^{\text {th }}$ order RungeKutta method for time-stepping of the governing equations. In all simulations we used: $a_{0}=50, \beta=50, \chi=0.4, \gamma=$ $0.25, \rho_{\min }=0.05, n_{L}=1, k=1.5$. Three simulations were done to elucidate different aspects of the dynamics. In the first, tip proliferation was included $\left(a_{1}=10\right)$, and we assumed a low TAF decay rate $(\lambda=1)$ and a low threshold for EC proliferation $(\vartheta=0.2)$ (Figure 9(a)). In the second (Figure 9(b)) EC proliferation was also active $\left(a_{1}=10\right)$. However, we considered high TAF decay rate $(\lambda=10)$ and high threshold for EC proliferation $(\vartheta=0.5)$. Finally, in the third simulation, we considered inactive EC proliferation $\left(a_{1}=0\right)$ and low TAF decay rate $(\lambda=1)$ (Figure 9(c)).

In all cases, the TAF dynamics are very fast, and when the TAF decay rate is low (Figure 9(a) and (c)) the tumor becomes vascularized, as judged by the fact that tip cells reach the boundary. In contrast, when the TAF decay rate is high vascularization is prevented (Figure 9(b)). Also, in both cases where vascularization is achieved, the maximum of the tip density leads that of the vessel density, which suggests that this model can capture the brush-border effect. Despite the fact that the model can correctly predict the effect of the TAF decay rate on vascularization, it also predicts that vascularization is possible even if EC proliferation is absent (Figure 9 (c)), which contradicts experimental observations that show EC proliferation is necessary for the vasculature to reach the tumor. 

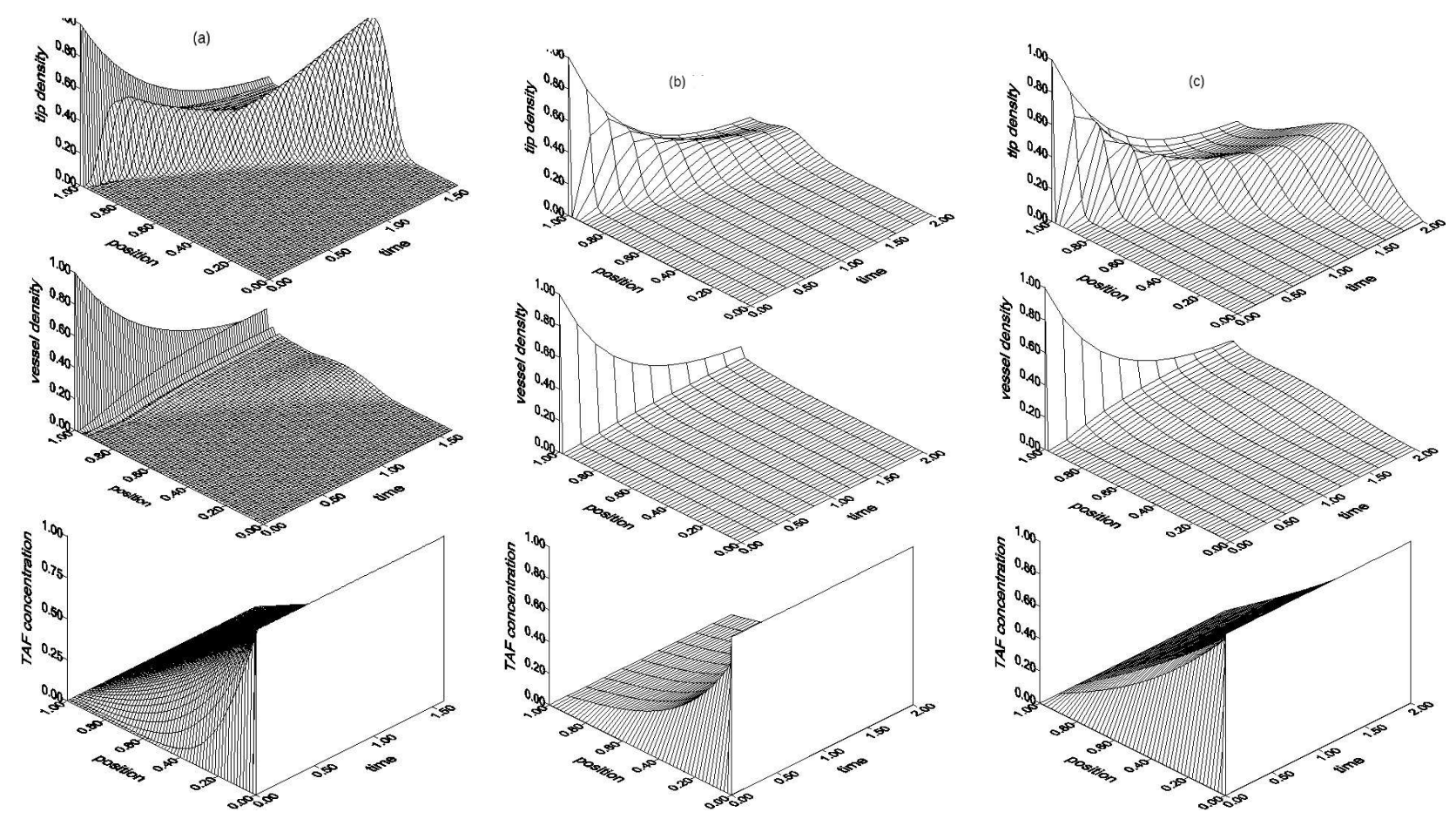

Figure 9: The Balding-McElwain as modified in Byrne \& Chaplain (1996): (a) EC proliferation, low TAF decay rate and low proliferation threshold, (b) EC proliferation, high TAF decay rate and high proliferation threshold, (c) No EC proliferation, low TAF decay rate.

However there are difficulties if the model is extended to two or three space dimensions. Firstly it is not clear how to generalize the source of new vessel cells, since the first two terms in (21) represent a flux in one dimension, but this would be replaced by a vector quantity in higher dimensions, projected onto a particular direction. Moreover, despite the fact that sprout ECs are active participants in anastomosis and branching events, as reflected in the tip density equation, this is not reflected in (21). Despite these shortcomings, this model has some rather unique features, since it is the only continuum model to explicitly account for (a) the localization of proliferation behind the moving tip, (b) branching as a process by which new sprouts are created from capillary cells, $m$ and (c) anastomosis as a process by which tip cells from one sprout merge with capillary cells from another sprout.

Orme \& Chaplain (1997) and Chaplain \& Orme (1998) developed a mathematical model describing the dynamics of endothelial cell density and the concentrations of TAF and fibronectin in two space dimensions. The novelty of their model lies in the incorporation of haptotaxis, which is modeled similarly to chemotaxis. In haptotaxis, the endothelial cell population migrates up the concentration gradient of fibronectin, which mediates cell-to-cell and cell-to-matrix adhesion. The TAF concentration is assumed to be at steady state. Thus, the resulting system of equations consists of just the endothelial cell number balance and the fibronectin mass balance. The model was simulated to examine the relative importance of various parameters, such as the chemotactic and haptotactic coefficients, the endothelial cell proliferation rate, the diffusion coefficient etc., on the dynamics of the angiogenic process. Without haptotaxis, the model predicts early anastomosis of the initial sprouts, and the authors use this result to deduce that haptotaxis is important for successful angiogenesis. This work offers qualitative and quantitative insight into the importance of the various processes considered. However, their results predict that the fibronectin concentration is the highest at the parent vessel and lowest at the tumor, and remains basically uniform along the x-axis. On the other hand, it is now known that in order for ECs to move, they must locally degrade the extracellular matrix, which contains fibronectin. This leads to the question as to how the EC move, since the fibronectin concentration remains very high.

Anderson \& Chaplain (1998a) later developed a one-dimensional continuum model to describe tumor angiogenesis in the absence of cell proliferation. In addition to diffusion and chemotaxis, the model included the process of haptotaxis. The chemotactic sensitivity was taken to be inversely proportional to the concentration of TAF to describe their hypothesis of TAF receptor desensitization at high TAF concentrations. This work mainly aimed at explaining the experimentally observed fact that in the absence of endothelial cell proliferation, angiogenesis is incomplete, in the sense that the EC fail to reach the 
tumor. The model described the dynamic interaction of ECs, TAF, and fibronectin. To simplify the analysis, fibronectin and TAF were considered to be at steady state. This model was later analyzed by Sleeman et al. (1999) to find the regions of the parameter space where angiogenesis will be complete and incomplete for given fibronectin and TAF steady-state profiles. The model is based on the hypothesis that the inability of the EC to reach the tumor in the absence of proliferation is due to endothelial cell desensitization to chemical gradients at high tumor angiogenic concentrations. Despite the possibility that there might be some truth into this hypothesis, the interpretation of the phenomenon must be significantly more complicated than that, since the use of this particular functional form of the chemotactic sensitivity in combination with the steady-state assumption for the TAF and fibronectin will predict a reduction in the speed of the vasculature as the tumor is approached, which is in contradiction with the findings of Folkman (1976).

Anderson and Chaplain extended the one-dimensional work to two space dimensions in order to obtain a more realistic picture of the angiogenic process (Anderson \& Chaplain, 1998b; Chaplain \& Anderson, 1999; Chaplain, 2000). As in the one-dimensional model, the 2D model does not contain any endothelial cell proliferation terms. As in the earlier work of Orme \& Chaplain (1997), the model has three unknowns: a) the EC density, b) the fibronectin concentration and c) the TAF concentration. The equations for the dimensionless endothelial cell density $n(x, y, t)$, dimensionless TAF concentration $c(x, y, t)$, and dimensionless fibronectin concentration $f(x, y, t)$, in a dimensionless square domain $[0,1] \times[0,1]$ (parent vessel at $x=0$ and tumor at $x=1$ ), respectively, are:

$$
\begin{aligned}
& \frac{\partial n}{\partial t}=\nabla \cdot\left[D \nabla n-n\left(\frac{\chi}{1+a c} \nabla c+\rho \nabla f\right)\right] \\
& \frac{\partial c}{\partial t}=-\eta n c \\
& \frac{\partial f}{\partial t}=\beta n-\gamma n f .
\end{aligned}
$$

The initial conditions used in all simulations are:

$$
\begin{aligned}
& n(x, y, 0)=e^{-\frac{x^{2}}{\varepsilon_{3}} \sin ^{2}(6 \pi y)} \\
& c(x, y, 0)=e^{-\frac{(1-x)^{2}}{\varepsilon_{3}}}
\end{aligned}
$$

or

$$
\begin{aligned}
& c(x, y, 0)=\left\{\begin{array}{cc}
1 & 0 \leq r \leq 0.1 \\
\frac{(v-r)^{2}}{(v-0.1)^{2}} & 0.1 \leq r \leq 1
\end{array} \quad \text { where } \quad r=\sqrt{(x-1)^{2}+\left(y-\frac{1}{2}\right)^{2}}\right. \\
& f(x, y, 0)=k e^{-\frac{x^{2}}{\varepsilon_{2}}}
\end{aligned}
$$

In both the one-and two-dimensional models the authors assume zero-flux boundary conditions on the ECs, which is somewhat unrealistic, since it is known that cells from the parent vessel migrate into the growing sprout. The values of parameters that appear in the equations (31)-(36), which were used in Anderson \& Chaplain (1998b) and in our simulations, are given in Table 1. We developed a numerical algorithm which uses a $2^{\text {nd }}$-order quadratic finite element algorithm for space discretization

Table 1: Model parameter values for the Anderson \& Chaplain (1998a) model.

\begin{tabular}{|c|c|}
\hline$D=3.5 \times 10^{-4}$ & $\nu=\frac{\sqrt{5}-0.1}{\sqrt{5}-1}$ \\
\hline$\chi=0.38$ & $\varepsilon_{1}=0.45$ \\
\hline$\alpha=0.6$ & $\varepsilon_{2}=0.45$ \\
\hline$\rho=0.34$ & $\varepsilon_{3}=0.001$ \\
\hline$\beta=0.05$ & $\kappa=0.75$ \\
\hline$\gamma=0.1$ & $\eta=0.1$ \\
\hline
\end{tabular}

and the unconditionally stable trapezoidal rule for time integration to study various predictions of the model, including the effects of different tumor geometries (a line tumor as reflected in the initial condition (35) vs a circular tumor, defined by (36), 

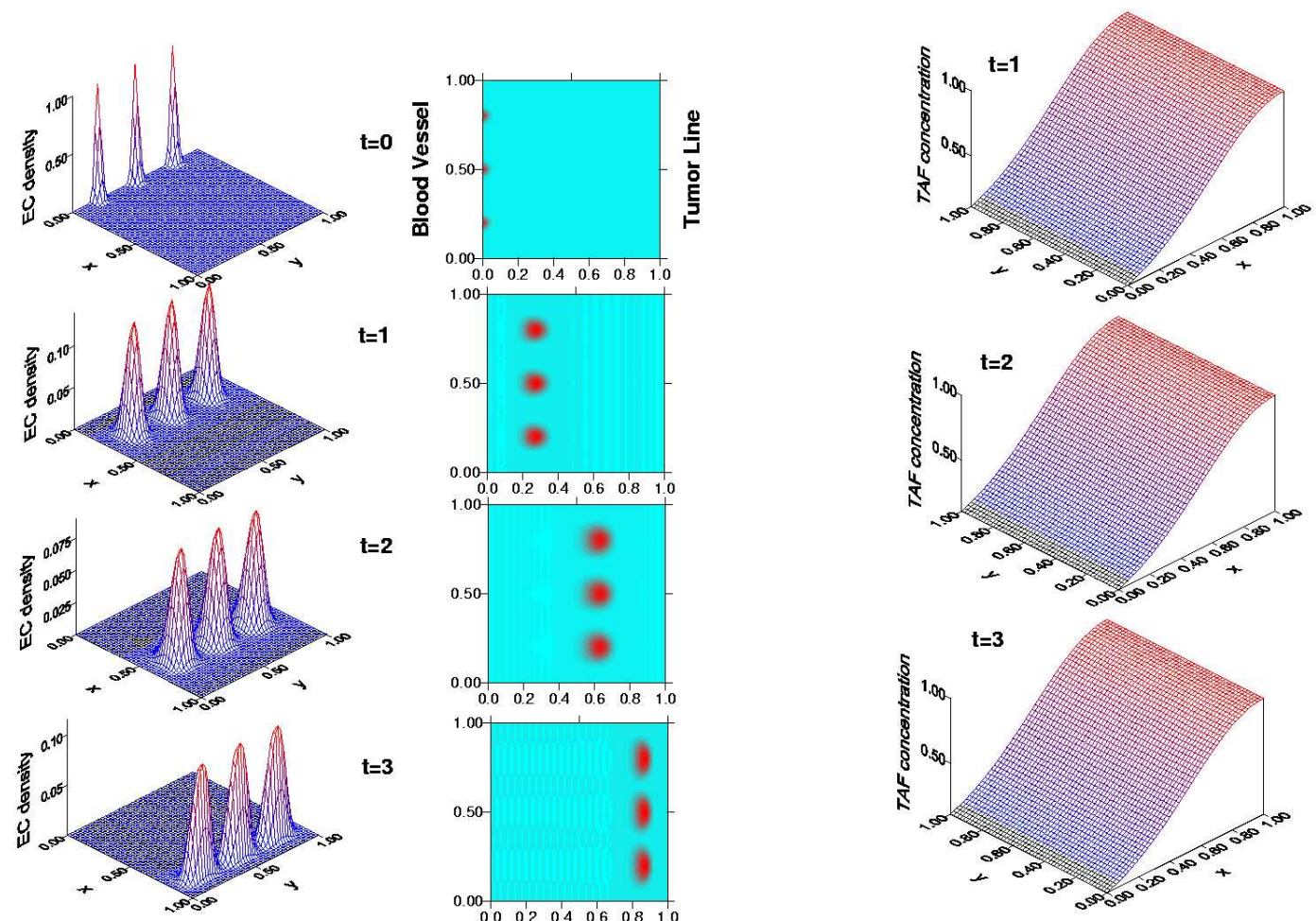

$=1$
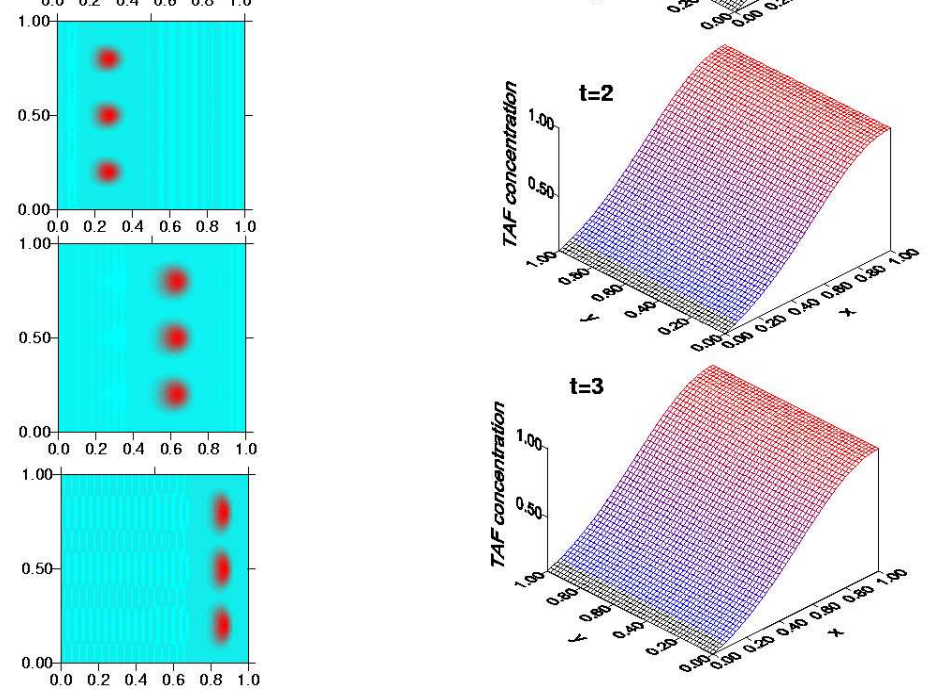

Figure 10: Two-dimensional spatial profiles computed using the model of Anderson and Chaplain (1998) without haptotaxis, for a line tumor initial condition. EC cell density (left) and TAF concentration (right).

as well as the effects of haptotaxis. In the first simulation (Figure 10) we assumed a line tumor (35) and excluded haptotaxis, in the second (Figure 11(a)) we also assumed a line tumor (35) and included haptotaxis, while in the third simulation we included haptotaxis, and assumed a circular tumor (36).

Notice that the TAF concentrations in Figure 10 do not change significantly with time (the same is true of the fibronectin concentration; results not shown) for the assumed parameter values. Despite the fact that there is no proliferation in the model, it predicts that the vessels reach the tumor in some cases, which contradicts the results of Ausprunk \& Folkman (1977) discussed earlier. Notice also that haptotaxis slows down the angiogenic process to the point where vascularization is not achieved in both the cases of the line and circular tumor. Furthermore, in the cases where successful angiogenesis is predicted, the results show that the speed of the vasculature front decreases as the distance from the tumor decreases, which contradicts the experimental observation of vasculature acceleration as the tumor is approached (Folkman, 1976). It can be attributed to the same reasons that apply to the one-dimensional model.

As was discussed earlier, angiogenic inhibitors represent a fundamental component of the angiogenic process, which is absent in this model and earlier continuum models for the dynamics of angiogenesis. This can be attributed to the fact that endostatin, the most potent inhibitor, was only discovered in 1997 and very little was known about other inhibitors when earlier models were developed. In a later paper Anderson et al. (2000) model the phenomenon of concomitant resistance, whereby an existing tumor secretes an inhibitor that prevents vascularization of other tumors. The model is one-dimensional and consists of three unknowns: EC density, and TAF and inhibitor concentrations. The inhibitor is modeled as second chemotactic species. As in their earlier work, it is assumed that the dynamics of TAF and inhibitors are very fast when compared to those of the EC, and thus these quantities are at steady state. The dynamics of the EC density are studied for various steady-state profiles for TAF and inhibitors, and the authors show weak, complete, and no vascularization under various conditions.

In addition to suggesting an explanation for the prevention of vascularization in a secondary tumor in the presence of a primary tumor, the paper aims to quantify the relationship between the extent of inhibition of the secondary tumor vascularization and the size of the primary tumor. This is achieved through the boundary condition for the inhibitors at the parent vessel, where it is assumed that the concentration is proportional to the size of the primary tumor. The authors assume zero inhibitor flux at the secondary tumor, but it is not explained why the secondary tumor does not secrete inhibitors if the primary 

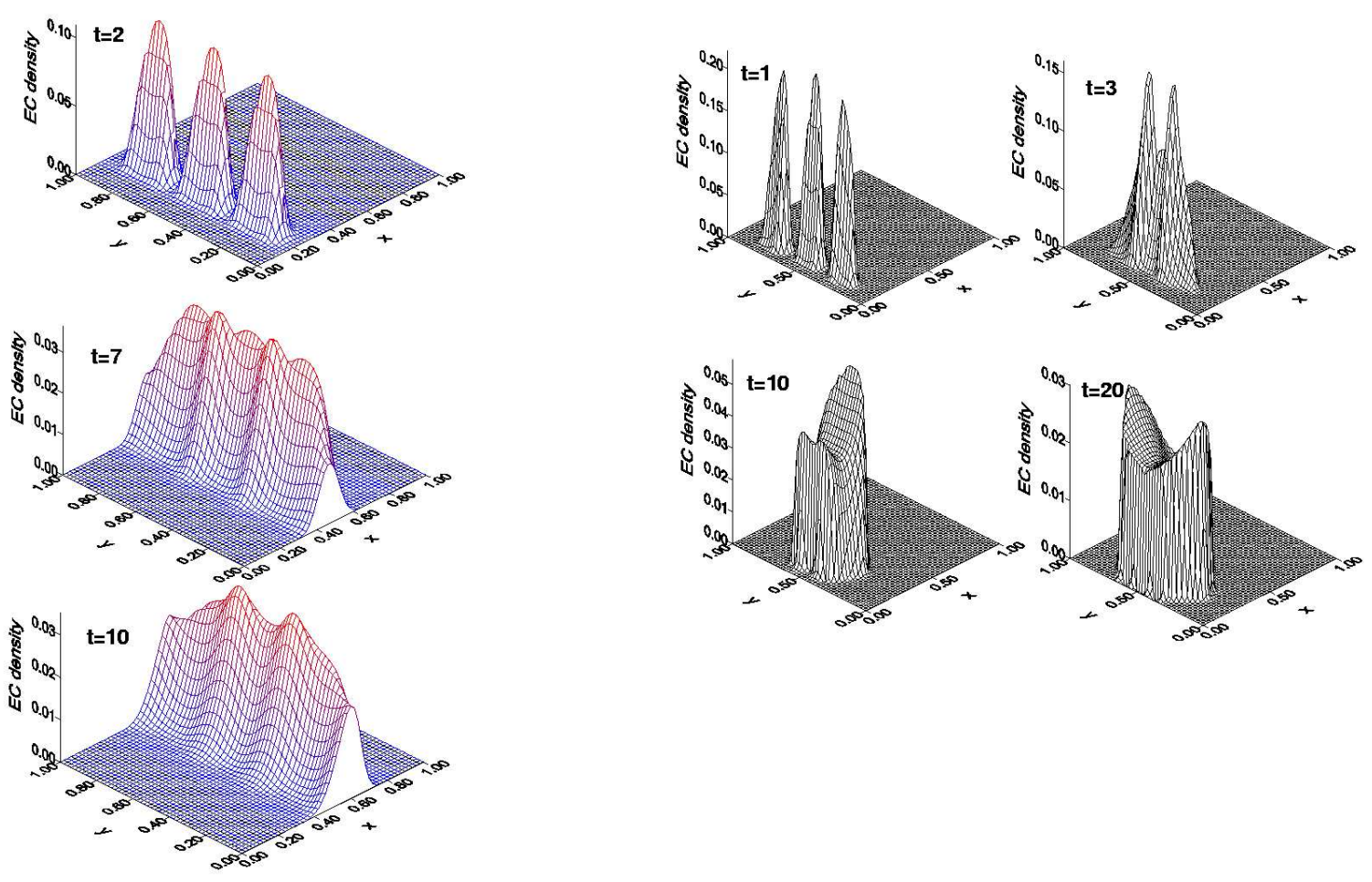

Figure 11: Two-dimensional spatial profiles of EC cell density computed using the model of Anderson and Chaplain (1998), including haptotaxis and line tumor (left) and circular tumor (right).

tumor does, as is assumed in their model. They further assume that there is no endothelial cell proliferation, but in some cases predict complete vascularization of the secondary tumor nonetheless.

However, the most significant conceptual difficulty with this model can be explained as follows: The chemotactic sensitivity of ECs for the inhibitor is taken to be positive, i.e. cells move up the gradient of the inhibitor concentration. In the case of concominant resistance, the high inhibitor concentration is close to the parent vessel of the secondary tumor. As a result there is no vascularization for inhibitor concentrations above a certain threshold at the parent vessel. However, one can also apply the model to describe angiogenesis at the primary tumor at an earlier stage, since the mechanisms are the same. In the case of primary tumor angiogenesis, there exist no inhibitors at the parent vessel because the inhibitors are secreted by the tumor according to Folkman's angiogenic switch hypothesis, which the authors adopt, and there are no vessels, at least at the outset. The primary tumor secretes inhibitors, so the inhibitor gradient is now in the opposite direction (inhibitor concentration is high at the tumor and low at the parent vessel) compared with that at a secondary tumor. In this case the model would predict that the inhibitors actually enhance EC migration toward the tumor.

The role of inhibitors, particularly angiostatin, during angiogenic outgrowth was also investigated by Levine et al. (2001a). They have developed a continuum model that builds on their previous work (Levine et al., 2001b; Levine et al., 2000) discussed earlier, to model the spatio-temporal course of angiogenesis from initiation at the primary vessel to the time at which the tumor is reached. In Levine et al. (2001a) the one-dimensional model for the onset of angiogenesis was combined with a two-dimensional model describing the dynamics of angiogenesis. This is a very complex model requiring more than sixty parameters. It contains five species: TAF, the protease enzymes, fibronectin, angiostatin, and EC. It consists of the one-dimensional equations describing the onset of angiogenesis at the parent vessel and the corresponding two-dimensional equations describing sprout formation in the tissue between the parent vessel and the tumor. The two sets of equations are coupled through the boundary conditions at the parent vessel. The biochemical kinetics included in both sets of equations are essentially taken from their earlier work (Levine et al., 2001b; Levine et al., 2000) with the exception of the protease enzyme production kinetics, which here contains a saturation term. In addition, decay of several factors such as the protease are included.

The complex structure of the extracellular matrix is accounted for using a single parameter (the porosity constant), which leads to a non-Fickian expression for the diffusive flux, suggested elsewhere for diffusion through porous media. The equa- 
tions that are used to model angiogenesis dynamics contain diffusion of TAF, fibronectin and angiostatin, as well as EC proliferation. Not all of these processes are included in the corresponding equations describing angiogenesis initiation. It is assumed that endothelial cell proliferation in the tissue depends only on the protease enzyme dynamics. No explicit dependence of the proliferation on TAF is considered, despite the fact that VEGF stimulates EC proliferation via a pathway that is regulated independently from the pathway responsible for EC migration (Koolwijk et al., 1998). According to this assumption, in order for cells to proliferate, the protease enzyme concentration should be above a certain threshold value. High values of the protease enzyme also stimulate migration through the degradation of fibronectin. Thus, in this model, proliferation and migration are tightly related, which contradicts some experimental observations. Furthermore, the authors account for localization of proliferation behind the leading tip of the vasculature by multiplying the proliferation term with an arbitrary function depending on tip curvature. The inclusion of this function, in addition to an adjustment of parameter values, accounts for the fact that their results partially reproduce this well-known feature of tumor-induced angiogenesis (Sholley et al., 1984). However, the experimentally observed phenomenon cannot be understood using this phenomenological approach; it can only be accounted for.

The boundary conditions at the parent blood vessel and tumor appear somewhat arbitrary. It is unclear whether the suggested boundary conditions at the parent vessel satisfy continuity of flux and concentration for the TAF and angiostatin. The boundary condition for the EC density at the parent vessel is based on the implicit assumption that in order for the EC to move within the tissue, the fibronectin concentration should drop below a certain threshold, thus reflecting the degradation of the basal lamina, which is necessary for EC invasion into the tissue. The authors postulate that the flux of EC at the tumor side of the domain is proportional to the EC density there, but this has not been confirmed experimentally, and is at least partially responsible for the prediction that the EC vasculature seems to accelerate as it gets closer to the tumor. Again, the model appears to include and not predict this experimentally-verified feature (Folkman, 1976). Finally, a puzzling feature of their numerical computations lies in the prediction of the time evolution of the fibronectin profiles. Fibronectin concentration seems to remain significantly low even behind the growing vasculature. If this is so, then what preserves the integrity of the vasculature behind the growing tip?

\subsubsection{Mechanochemical Models}

Continuum models based on formulations such as (1)-(4) only incorporate the chemical interactions between the EC and the extracellular matrix (ECM), and then only partially because the complex fibrous structure of the ECM is not explicitly included in such models. Fibronectin is usually the "chemical representative" of the ECM and the incorporation of its mass balance is considered to account for EC-ECM interactions in conventional continuum models. However, as was discussed earlier, EC-ECM interactions are not only chemical but also mechanical, since the EC cells exert traction forces on the ECM fibers in order to move. These interactions were incorporated into mechano-chemical models introduced by Oster and coworkers (Oster et al., 1983; Murray et al., 1983) to offer an alternate explanation to the Turing theory for pattern formation in early development. A typical mechano-chemical model retains the basic structure of model (1)-(3), but augments the EC flux (3) by a convective term that models transport of cells and ECM due to local deformation in the ECM Murray \& Swanson (1999). This additional flux arises from the fact that forces exerted on the ECM by other cells induce passive movement of cells on the ECM. This additional convective component of (3) is

$$
J_{\text {con }}=n \frac{\partial u}{\partial t}
$$

where $u$ is the ECM displacement from an initial position. The displacement is in turn determined from the momentum balance equation for the ECM, in which inertial effects are neglected by virtue of the slow motion of the ECM compared to that of the cells. Thus

$$
\nabla \cdot \sigma+\rho F=0
$$

where $\sigma$ is the stress tensor and $F$ is the external force per unit mass acting on the matrix. $\sigma$ comprises both cell and ECM components, and is written in the additive form

$$
\sigma=\sigma_{\text {cell }}+\sigma_{\text {matrix }} .
$$

It is postulated that the contribution of EC to the stress tensor will increase with increasing cell density at low densities, but will decrease at high cell densities due to cell-cell inhibition, and this is captured in the expression

$$
\sigma_{\text {cell }}=\frac{\tau n}{1+\lambda n^{2}} .
$$

The ECM is treated as a viscoelastic material, and hence its contribution to $\sigma$ is $\sigma_{\text {matrix }}=\sigma_{\text {viscous }}+\sigma_{\text {elastic }}$. Deformations are assumed to be small and therefore the viscous part can be taken proportional to the rate of change of the elastic strain tensor 
$\varepsilon=\frac{1}{2}\left(\nabla \cdot u+\nabla \cdot u^{T}\right)$ and the rate of change of the dilation $\theta=\nabla \cdot u$ of the ECM. Thus:

$$
\sigma_{\text {viscous }}=\mu_{1} \varepsilon_{t}+\mu_{2} \theta_{t} I
$$

where $I$ is the unit tensor and $\mu_{1}$ and $\mu_{2}$ are the bulk and shear viscosities of the ECM, respectively. Finally, the elastic component of the matrix contribution to the stress tensor is given by:

$$
\sigma_{\text {elastic }}=\frac{E}{1+\nu}\left(\epsilon+\frac{\nu}{1-2 \nu} \theta I\right)
$$

The force $F$ is usually taken to be proportional to the displacement and written in the form $F=-s u$. The model is completed with the addition of the ECM mass balance, the density $\rho(x, t)$ of which satisfies

$$
\frac{\partial \rho}{\partial t}=-\nabla \cdot\left(\rho \frac{\partial \mathrm{u}}{\partial t}\right)+S
$$

As (44) shows, the motion of the matrix is assumed to be only due to convection, while $\mathrm{S}$ represents the net effect of production and destruction of the ECM. S is typically taken to be negligible in most mechanochemical models using a time scale argument, which may or may not be accurate.

In summary, a typical mechanochemical model consists of (1)-(3) with the flux (Eqn 3)) augmented by the convective flux (38), (44) for the ECM density and (39) for the ECM displacement. For the displacement, zero boundary conditions are typically specified on the entire boundary, while for ECM density zero flux is naturally assumed. Finally, the phenomenon of contact guidance, where the ECM defines the preferred direction of cell motion, is taken into account through the dependence of the EC diffusion coefficient on the elastic stress tensor.

Holmes \& Sleeman (2000) developed such a mechanochemical model to study the role of the extracellular matrix in a more direct way by including the dynamics of the ECM displacement in the model and by considering the ECM to be an active participant in the process. The two-dimensional continuum model that they proposed is a combination of the two-dimensional work of Chaplain and co-workers ((Chaplain et al., 1995; Chaplain \& Anderson, 1996; Orme \& Chaplain, 1997)) (with the addition of EC proliferation) and the mechano-chemical model developed by Murray and co-workers (Murray \& Swanson, 1999; Manoussaki et al., 1996; Murray \& Oster, 1984). The model consists of balances on TAF, fibronectin, extracellular matrix, and EC. It does not include any angiogenic inhibitors. No-flux boundary conditions are specified for the EC density and fibronectin on the entire boundary. Dirichlet boundary conditions are assumed for the TAF concentration at the parent vessel and the tumor ( 0 and 1, respectively) and no flux elsewhere. The system has spatially uniform and spatially nonuniform steady states. The authors linearized the equations around a temporally stable uniform steady state and performed Turing-type linear stability analysis. This analysis revealed a critical traction number, which was said to quantify the influence of the ECM on EC motility. Above this critical traction number diffusion-driven instability is observed. The authors then performed various numerical simulations with the full nonlinear model in order to illustrate the patterns observed in the two regions of the parameter space (below and above the critical traction number). Above the critical traction number, the authors present more heterogeneous structures. Below this number more diffuse structures are obtained although the differences are not dramatic. The model represents an attempt to actively investigate the role of the extracellular matrix in the angiogenic process. In that respect, it represents a step towards the positive direction, although it is possible that such an investigation can perhaps be performed with the development of a simpler model.

\subsection{Discrete Models}

Despite the fact that continuum models can provide significant insight into the relative role that different process play in the formation of vascular structures, they cannot actually predict the vascular structures. In order for a model to predict the treelike structures that sprouts form, the model should recognize the existence of separate sprouts. This distinction is not present in any of the continuum models discussed earlier. Various discrete models such as fractal models, inverted diffusion limited aggregation (DLA) models, L-systems, lattice models etc. have been presented in the literature ((Kiani \& Hudetz, 1991; Landini \& Misson, 1993; Gazit et al., 1995; Baish et al., 1996; Nekka et al., 1996)) to explain and describe the branching morphology of the vascular networks.

One of the first and most successful models to approach the problem using a discrete framework was that of Stokes \& Lauffenburger (1991). This model is discrete in the sense that it treats sprouts individually and tracks the motion of the tip of a growing sprout in two space dimensions. Each sprout is characterized by the position and velocity of its tip cell at a given point in time, and the average density of sprouts is computed. The evolution of the tip velocity is governed by a stochastic 


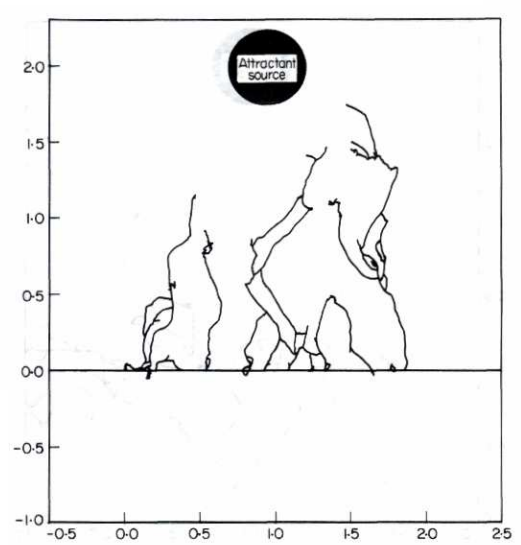

Figure 12: The two-dimensional spatial distribution of sprouts predicted by simulation of the Stokes-Lauffenburger (1991) model. The primary vessel is represented by the horizontal line and units are in millimeters. For parameters see Stokes \& Lauffenburger (1991); figure reproduced with permission.

differential equation that comprises a viscous damping term, a white noise term to model random motion, and a chemotactic component. The resulting equation is

$$
d v_{i}(t)=-\beta v_{i}(t) d t+\sqrt{\alpha} d W_{i}(t)+\kappa \nabla a \sin \left|\frac{\Phi_{i}}{2}\right| d t
$$

where $\beta$ is a 'viscosity' coefficient, $W$ is the Wiener process, a white noise process, $\kappa$ is the chemotactic responsiveness, $a$ is the TAF concentration, $\Phi$ is the angle between the direction the tip is moving and that towards the TAF source. The subscript $i$ denotes the $i^{t h}$ sprout. The equation for the tip position is the deterministic equation:

$$
\frac{d x_{i}(t)}{d t}=v_{i}(t)
$$

Finally, the equation for the average density is written

$$
\frac{d \rho_{i}(t)}{d t}=k_{g} \frac{\rho_{\max }-\rho_{i}(t)}{\rho_{\max }-\rho_{\min }} \rho_{i}(t)-\frac{s_{i}(t)}{L_{i}(t)} \rho_{i}(t)+k_{b}\left[\rho_{p}(t)-\rho_{i}(t)\right]-\sum_{j=1}^{\nu_{i}} k_{b}\left[\rho_{i}(t)-\rho_{j}(t)\right]
$$

where $\mathrm{k}_{g}$ is the proliferation rate, $\mathrm{k}_{b}$ is the redistribution coefficient, $\rho_{p}(\mathrm{t})$ is the parent vessel density, $\nu_{i}$ is the number of branches that the $\mathrm{i}^{t h}$ sprout has at time $\mathrm{t}, \mathrm{s}_{i}$ is the instantaneous speed of the $\mathrm{i}^{\text {th }}$ sprout calculated as $s_{i}(t)=\left\|\mathrm{v}_{i}(t)\right\|$ and $L_{i}$ is the sprout length calculated from:

$$
\frac{d L_{i}(t)}{d t}=s_{i}(t)
$$

The first term of (47) describes EC proliferation using a logistic expression. The second accounts for sprout elongation using an average velocity and an average length. The third term describes the rate of increase of the average density of the $\mathrm{i}^{\text {th }}$ sprout due to influx of ECs from the parent vessel, while the fourth term is the rate of loss of density due to the redistribution of EC from the $\mathrm{i}^{\text {th }}$ sprout to each of its $\nu_{i}$ branches.

At each time step these three ODEs are solved for each sprout. The total number of sprouts changes due to branching (birth) and anastomosis (death of sprouts). Due to the discrete nature of the model, the authors account for anastomosis in the most natural way: when a sprout tip comes within the distance of a single cell from another sprout (either tip or main body), the sprout tip vanishes and its velocity is set to zero. Branching is accounted for using a probability. For the chemoattractant TAF, a certain steady-state profile is assumed. A typical result of the simulations is shown in Figure 12.

Besides naturally describing anastomosis, the model is also able to predict what can be experimentally visualized, i.e. the movement of individual sprouts. Moreover, it can provide insight into the heterogeneity of the tumor vascular network, which is believed to be of crucial importance in understanding tumor angiogenesis. These features are simply not present in the existing continuum models. In this respect, this paper is unique in the angiogenesis literature. Finally, the authors take into account the influx of EC from the parent vessel in the growing vasculature and from a parent sprout into its branches 
through a redistribution term. Although such an assumption is perfectly logical is notably absent from the majority of the other mathematical models presented to describe angiogenesis. Although the model as described is for two space dimensions, it can easily be extended to three space dimensions.

On the other hand, due to the representation of the sprout density by an average (along the sprout length), sprout elongation and cell proliferation cannot be accounted for properly, since both processes are localized close to the tip of the sprout. Furthermore, the incorporation of branching through a probability, which increases as the tumor is approached, is purely phenomenological. Moreover, the model does not include any angiogenic inhibitors, which is normal however, since the most important angiogenic inhibitors were discovered after the publication of this article. Also, their simulations fail to predict this pronounced branching phenomenon as the tumor is approached (brush-border effect). Finally, as in the majority of the continuum models described earlier, the dynamics of TAF concentration were assumed to be at a steady state, which has not yet been experimentally verified. It is conceivable that as soon as neovascularization is initiated, the loss of TAF from binding on the EC could significantly alter the TAF profile. This question of the role of TAF dynamics is addressed by Tong \& Yuan (2000) who use a model very similar to that of Stokes and Lauffenburger but relax the steady-state assumption for the TAF. Their results reproduce experimental measurements in the rat cornea well, which the authors attribute to the incorporation of TAF dynamics in the model.

Anderson \& Chaplain (1998b); Baum et al. (1999); Chaplain \& Anderson (1999); Chaplain (2000) have presented a completely different discrete model than the one that Stokes and Lauffenburger suggested. The first version of this model was presented in the form of a deterministic cellular automaton (DCA) model (1996). They derived it by applying the inverse of the procedure that Othmer \& Stevens (1997) applied. The latter authors started from various discrete models (local, gradient, nearest neighbor) for cell motion and then went to the continuum limit. On the other hand, Anderson and Chaplain started by discretizing a two-dimensional continuum model using $2^{\text {nd }}$ order finite differences and explicit Euler and used this discrete form to assign probabilities of cells moving to different grid points at the next time step. These probabilities obviously contain contributions from diffusion, chemotaxis, and haptotaxis. Then, this model was augmented to include anastomosis, branching and proliferation, which weren't present in the continuum model. The model was used to visualize sprout growth and vascular network formation in two and three spatial dimensions. The model results are similar in morphology and structure to vascular networks observed in vitro.

Similar to Stokes and Lauffenburger, branching is accounted for phenomenologically, through the assignment of a probability, which is assumed to increase as the tumor is approached. The authors formulate anastomosis rules that resemble those of Stokes and Lauffenburger. Anderson and Chaplain's rules are more complex since they attempt to account for the observation that anastomosis starts to occur some definite distance away from the parent vessel ((Paweletz \& Knierim, 1989)). However, again the model does not seem to offer any explanation for this observation. It simply incorporates it. Finally, EC proliferation is included only behind the sprout tip, which was not possible in the model of Stokes \& Lauffenburger (1991).

\section{Summary and remarks}

The inescapable conclusion to be drawn from the foregoing is that angiogensis is a very complex process comprised of many subprocesses, each of which will require mathematical modeling in order to make quantitative predictions about the dynamics. In this last section we provide a brief summary of the overall process, we speculate on some issues raised by experimental observations, and suggest a number of key steps that we believe are currently amenable to modeling.

In response to hypoxia, tumor cells secrete VEGF which diffuses into the surrounding tissue. The ECs in the neighboring parent vessel that are nearest this stimulus begin to secrete proteolytic enzymes, such as PA-plasmin and MMPs, which dissolve the basal lamina of the parent vessel and the surrounding ECM. This cleavage has positive and negative effects. Firstly, it facilitates the movement of the cells towards the tumor. Secondly, it mobilizes VEGF, bFGF and other angiogenic factors from the ECM. These factors rapidly induce PA-plasmin production in ECs, stimulating further invasion through the surrounding ECM. VEGF and bFGF also stimulate proliferation of the ECs located close to the moving front of the neovascular network where the ECM is more damaged. Thirdly, it stimulates the mobilization of fibronectin, which helps in the adherence of neighboring cells by the process of haptotaxis. Fourthly, the proteases further cleave plasminogen, collagen, and fibronectin, thereby releasing potent angiogenic inhibitors which locally inhibit either proliferation or chemotaxis (or both) of ECs, or they may even act as chemorepellants leading to phenomena such as anastomosis. Furthermore, plasmin activity induces the release and activation of LTGF- $\beta$ from the basement membrane. The active TGF- $\beta$ counteracts the effects of bFGF/VEGF on ECs by downregulating uPA and MMP expression and by stimulating PAI-1 and TIMP synthesis. As a result, plasmin formation is blocked and pro-MMPs are no longer activated. Thus, ECM proteolysis is locally inhibited, which reduces EC migration. During this stage, it is possible that ECs can re-associate with each other and form interconnected tubelike structures with intra- and intercellular canals and lumens. However, the proteolytic blockade also turns off the plasmin- 
mediated activation of LTGF- $\beta$. With less active TGF- $\beta$, PAI- 1 synthesis will fall, the effects of bFGF and VEGF on uPA and UPAR synthesis again become prevalent and the PA proteolytic activity increases. ECs at the tip of the capillary sprout invade from this location and a new elongated capillary sprout develops. Thus capillary lumina formation can be thought of as resulting from alternating cycles of growth factor/inhibitor- driven activation and inhibition of extracellular proteolysis and cellular proliferation. Throughout the angiogenic process there is a competition between the positive and negative effects that protease secretion, stimulated by growth factors such as VEGF, has on angiogenesis.

The ECM is a vast reservoir of latent growth factors, such as bFGF and VEGF, that are released via the action of PAplasmin and MMP exponents. This proteolytic cascade has the effect of constitutively increasing the local concentraion of growth factors, which can promote EC proliferation and subsequent invasion through a compromised ECM. But, what is the appropriate balance of growth factors required for capillary lumen formation? Studies by Montesano et al. (1990), in which monolayers of ECs on three dimensional gels were treated with either bFGF or both bFGF and TGF- $\beta$, have addressed this question. When added alone, the cells invaded the underlying gel, resulting in the formation of branching tube-like structures with widely patent lumenia. However, in response to co-added bFGF and TGF- $\beta$, both invasion potential and lumen diameter of the capillary like tubes was markedly reduced, and at concentrations of $5 \mathrm{ng} / \mathrm{ml} \mathrm{TGF}-\beta$ the cells migrated out as solid endothelial cell cords, i.e., lumen formation was completely inhibited. Since the magnitude of PAI-1 induction is proportional to TGF- $\beta$ concentration, one would expect TGF- $\beta$ induction to reduce the invasive potential of the migrating ECs, but what accounts for the reduction in lumen diameter? The observation that the capillary lumen is devoid of ECM material suggests that lumen formation is a proteolysis-driven event. Thus, the anti-proteolytic effect of TGF- $\beta$, which results from a large increase in PAI-1 and a subsequent reduction in fibrinolysis, probably accounts in part for the observed reduction in lumen diameter. Note that more physiologically realistic lumen diameters were seen at $500 \mathrm{pg} / \mathrm{ml}$ TGF- $\beta$ than were seen in the absence of TGF- $\beta$, which demonstrates directly that the key event in normal capillary morphogenesis is the maintenance of a protease-antiprotease equilibrium, rather than an increase in VEGF- or bFGF-mediated proteolysis.

The observation that angiogenesis reflects a perhaps delicate balance between competing influences leads to a number of questions concerning the outcome when one of the processes dominates. For instance,

1. What happens when there is too much proteolysis? An in vitro model of capillary lumen formation has been developed which consisits of endotheial cell tumors (hemangioma-derived or mT-transduced ECs) in suspension in threedimensional fibrin gels (Montesano et al., 1990). The mT-expressing ECs have a greater PA proteolytic activity than their normal counterparts and express very little PAI-1. In contrast to normal ECs, which frequently form a network of cell cords and tube-like structures under these in vitro conditions, when the mT-expressing ECs are embedded in fibrin gels they form large cyst-like structures which are lined by a monolayer of ECs. These structures bear stricking resemblance to the large cysts that are found in some endothelial tumors in vivo (Montesano et al., 1990; Dubois-Stringfellow et al., 1994). In these experiments, normal morphogenesis, i.e., the formation of capillary-like tubes, could be restored by the addition of broad spectrum serine protease inhibitors. These findings demonstrate that excessive PA-plasmin mediated proteolysis in mT-expressing ECs results in the formation of EC-lined cysts and is not compatible with normal capillary morphogenesis.

2. What happens when there is too much growth factor? Vernon \& Sage (1999) demonstrated that increased levels of VEGF favor the invasion of collagen ECM by single ECs rather than multicellular sprouts. The formation of EC capillary tubes in vitro or in vivo requires cell-cell adhesion via specific cell surface adhesion molecules, such as cadherin-5 or CD31 (Matsumura et al., 1997; Bach et al., 1998). Upon acquisition of a migratory, invasive phenotype, cells from a variety of tissues have been shown to decrease cadherin-mediated cell-cell adhesion Doki et al. (1993); Monier-Gavelle \& Duband (1995). Moreover, VEGF increases EC expression of several integrin surface receptors for ECM proteins such as collagen, fibronectin and vitronectin (Byzova et al., 2000; Lu et al., 1996; Senger et al., 1997). So why the invasion of single ECs versus multicellular sprouts? A possible explanation is simply that the VEGF-mediated increased EC-ECM adhesion out-competed the adhesion between neighboring ECs, with a consequence of enhanced single cell migration at the expense multicellular sprout formation. In conclusion, there must be an equilibrium between growth factors bFGF and VEGF and the antiangiogenic TGF- $\beta$ in order for lumen formation to occur. VEGF stimulates a migratory and invasive phenotype in ECs, but lumen formation on the fly is difficult to envision.

3. How can the balance be tipped in favor of EC-EC adhesion and lumen formation to give angiogensis? The first hypothesis attempting to address this question was suggested by Folkman and co-workers and is well known in the literature as the "Angiogenic Switch Hypothesis". According to this, the tumor cells produce both the angiogenic inducers and the protease enzymes that are responsible for the cleavage of the ECM resulting in the release of the inhibitors. When there is no angiogenesis, there is a balance between the inducers and the inhibitors in favor of the inhibitors, while when angiogenesis is observed the balance is shifted in favor of the inducers. Hypoxia is thought to be the primary reason for 
shifting the balance towards the vascular phenotype. Therefore, the angiogenic switch hypothesis essentially suggests that once the switch occurs, angiogenesis proceeds as a "slave" process, the regulation of which is independent of ECs.

On the other hand, only very recently, Wu et al. (2001) have shown using Northern and Western blot analyses that collagen XVIII-derived endostatin and TSP-1 are directly secreted by ECs as well as pericytes. In addition, ECs also secrete Ang-2. The EC expression levels of endostatin were higher than those of pericytes. Furthermore, Karihaloo et al. (2001) proposed that endostatin secreted by the local degradation of collagen XVIII in the ECM, plays the role of a local regulator, which acts to prevent unchecked ureteric bud outgrowth and branching. These two models differ from each other in that one suggests intracellular production of endostatin from the ECs, while the other suggests extracellular production of endostatin through the cleavage of the ECM. However, they both propose a more active involvement of non-tumor cells in endostatin production. Contrary to the angiogenic switch hypothesis, this idea suggests a more dynamic role of the inhibitors in the angiogenic process, since, according to this scenario, the release of angiogenic inhibitors occurs simultaneously with EC migration and proliferation.

To complicate things further, the supporters of the angiogenic switch hypothesis have suggested (Wen et al, 1999) that the generation of endostatin from collagen XVIII is a two-step process mediated by a matrix metalloproteinase and an elastase, while O'Reilly et al. (1999) showed that MMP-2 (gelatinase A) secreted by Lewis Lung Carcinoma primary tumor cells is responsible for the production of angiostatin. The findings about endostatin in particular, contradict the quantitative results that $\mathrm{Wu}$ et al. (2001) obtained.

However, the two existing theories may not be as different as they appear to be at first glance, if one considers that Wu et al. (2001) performed experiments using only ECs and pericytes, while Wen et al. (1999) performed experiments using only tumor cells. Furthermore, Wu et al. (2001) demonstrated that the expression levels of endostatin were significantly reduced under hypoxic conditions, which is in agreement with the angiogenic switch hypothesis where it is postulated that the switch in favor of the inducers is primarily the result of hypoxia. Therefore, it is possible that both of the suggested scenarios are true. Both tumor cells and ECs may be involved in the release of endostatin and similar angiogenic inhibitors in vivo. The question then becomes: which one of the two mechanisms is quantitatively the most dominant in vivo?

Both the continuum and discrete models have been able to account for many features of angiogenesis such as capillaryloop formation and the dynamics of blood vessels. However, with each of these models the process of sprout extension leading to the formation of a capillary network has been simulated by tracking the path of the leading capillary tip. These models are based on the hypothesis that the motion of the entire capillary sprout is governed by the motion of an endothelial cell located at the tip, and that the ECs which line the capillary sprout play the role of passive bystanders in the angiogenic process. It is now recognized that these cells are playing an active part in angiogenesis, including cytokine production, cellular adhesion and detachment, and lumen formation (Lowe et al., 1995).

Of course much remains to be done before a quantitative understanding of angiogenesis is possible, and to conclude, we identify a number of processes for which mathematical models can shed light on the interplay between different factors controlling these processes. We list these in order of chronological dependency, ordered by the progression of angiogenesis, and as the reader will note, the list is merely a re-synthesis of the material discussed earlier, but now with the emphasis on the underlying processes rather than the biochemical factors.

1. Tumor-vessel signaling Tumor cells produce a number of TAFs that stimulate a number of changes in ECs, and thus understanding the spatiotemporal distribution of the angiogenic factors is an essential component of modeling. This involves not only the physical process of molecular diffusion through the ECM, but also the details of the feedbacks between ECs and tumor cells, and of the presence or absence of secondary sources of TAFs, such as macrophages. Since TAFs stimulate EC proliferation, at least when the latter are sufficiently removed from the primary vessel, the question arises as to whether there is local inhibition of EC proliferation near the primary vessel, or whether a sufficiently high level of TAF suffices to induce proliferation. In addition the roles of factors such as Ang-1,2, Tie-2, and PDGF should be investiated, as well as desensitization of EC receptors to TAF (Hanahan, 1997).

2. Cell-based modeling of growth, proliferation, and movement Cell movement in a three-dimensional context in response to chemotactic attractants or repellents or changes in the ECM is a major process in angiogenesis and one for which there are no current models. The signal-transduction networks involved in the choice of direction are partially understood, but a complete understanding of how the various intra- and intercellular components interact can only come from quantitatively-accurate models. Modeling the dynamics of each individual endothelial cell of the newlydeveloped capillary vessel also has the advantage that one can incorporate processes such as mitosis, cell death and lumen formation. Once blood flow is restored, there is a period of vascular reorganization. Redundant capillaries 
regress, a process which requires cell death, whereas other capillaries develop into mature, quiescent vessels with increasing diameters and ECM deposition. The mechanisms of vascular maturation are unclear (Whalen and Zetter, 1992). Incorporation of cell death and vacuole development into a model can help identify the important factors involved in capillary regression and vasculature maturation. Dictyostelium discoideum is a model system for all aspects of cell motility and there are a number of models cell motion and morphogenesis in this system (Dallon \& Othmer, 1997; Savill \& Hogeweg, 1997).

3. Mechanisms for localization of growth behind the sprout tip EC proliferation is fundamental in order for EC to reach the tumor. In the absence of endothelial cell proliferation, a restricted capillary network that never reaches the tumor is formed (Sholley et al., 1984), although vascular sprouting can occur without EC proliferation. It has been observed experimentally that most of the EC proliferation is confined just behind the sprout tip. If EC proliferation is directly dependent on the concentration of TAF, then the higher proliferation rate of the tip ECs can perhaps be attributed to the fact that tip cells have more receptors exposed to higher TAF concentration than cells located behind the tip.

4. Anastomosis Anastomosis occurs more frequently further from the tumor, and it is observed at a certain distance away from the parent vessel, but in general, closer to the parent vessel than to the tumor (Paweletz \& Knierim, 1989). A possible explanation that can be tested with a mathematical model is that the inhibitors act as chemo-repellants for ECs. Very close to the parent vessel, inhibitor concentrations and gradients are small since their production is stimulated by TAFs, which also exist at low concentrations close to the parent vessel. Therefore, reverse or lateral migration of ECs leading to anastomosis is infrequent. However, at a distance from the parent vessel the TAF concentration is higher, which stimulates the release of more inhibitor as well. Thus the local gradients of inhibitor concentration become high enough to stimulate the lateral or reverse migration of EC, which leads to anastomosis. In this scenario, anastomosis is viewed as a result of a local "victory" of the inhibitors over the inducers, which can occur in moderate concentrations of TAFs. At higher TAF concentrations migration of EC towards the tumor will dominate over reverse migration, despite high concentrations of locally-released inhibitors.

5. Acceleration of the vascular network near the tumor Another experimental observation is that the speed at which the vascular network approaches the tumor increases as the distance from the tumor is decreased (Folkman, 1976). An hypothesis that may explain this rests on the interaction between the TAF and EC spatial profiles. As the vasculature approaches the tumor, cell proliferation becomes more significant, provided that the proliferation rate increases for higher TAF concentrations. Furthermore, TAF uptake by the cells, either by binding or due to binding and internalization, also becomes more pronounced as the cell density rises. As a result, the local gradient of TAF increases and since the chemotactic velocity is proportional to the TAF gradient and the EC density, the speed of propagation will increase. A model that produces more detailed information on the spatial profiles of TAF concentration and EC density could be used to test the plausibility of this explanation.

6. Branching in the growing network of capillaries Branching in the network occurs predominantly near the tumor, where new sprouts originate close to the tip subpopulation of existing sprouts. This produces the well-known brushborder effect, in which the network density increases significantly near the tumor (Gimborne et al., 1974; Ausprunk \& Folkman, 1977; Zawicki et al., 1981; Muthukkaruppan et al., 1982). The models described later treat branching in a phenomenological way, through the use of a branching probability. An hypothesis to explain this mechanistically goes as follows. Sprout formation is the result of the organized alignment of endothelial cells in an appropriate conformation, and alignment requires some time. As ECs approach the tumor, they encounter higher TAF concentrations, and if the proliferation rate increases with increasing TAF concentration, a large number of proliferating EC will accumulate very close to the tip of the sprout. Furthermore, since the local TAF gradient increases and the local EC density is higher closer to the tip, these new cells will move faster because of the stronger chemotactic effect. Therefore, either cells don't have enough time to align themselves with the existing sprout or the chemotactic forces are too strong and preclude this. In either case, they move in alternate directions, thus resulting in branching. A computational model could be used to test the validity of this and other hypotheses for the mechanism(s) responsible for branching.

7. Remodeling of the vasculature Tumor vessels are leaky and are being continually remodeled. Once the spatiotemporal distribution of the angiogenic factors such as VEGF, bFGF and TGF- $\beta$ are known during vessel outgrowth, one can incorporate Ang-1, Ang-2, and Tie-2 expression to understand where in the new network the vessels are leaky and where they're not. For example, high Tie-2/Ang-1 results in mature vessels that are not subject to remodeling, whereas high Tie-2/Ang-2 and high VEGF leads to vessel growth and leakiness, but high Tie-2/Ang-2 with little or no VEGF produces cellular apoptosis and vessel regression. Modeling maturation and remodeling of the vasculature can then be extended further by explicitly including pericytes and PDGF in the model. A first step is to understand PDGF expression in the growing vasculature, for this will determine (and predict) pericyte recruitment to the developing 
sprouts. One hypothesis is that PDGF secreted by the ECs attract the pericytes and also induces them to proliferate. Independently, the ECs and pericytes produce latent TGF- $\beta$, which is then activated in a plasmin-dependent manner once the ECs and pericytes make contact. The activated TGF- $\beta$ inhibits EC and pericyte proliferation, leading to maturation. Of course, this inhibition only takes place if there is Ang-1/Tie-2. It is known that pericytes exhibit a number of characteristics consistent with muscle cell activity and it has been proposed that they can regulate blood flow by transferring contractile forces via long processes to the EC capillary (Hirschi \& D'Amore, 1996). A more detailed picture of how various factors and processes interact in remodeling will give more detailed information about blood flow rates in the tumor, which may lead to better strategies for drug delivery.

8. The role of macrophages in angiogenesis Moldovan (2002) suggests that angiogenesis may depend heavily on macrophage infiltration. In this scenario, macrophages create a path for the capillary ECs as they migrate into the tissue as part of the immune infiltrate. Here EC migration is more 'path-following' and plays a secondary role in determining network structure driven by VEGF. Since macrophages respond chemotactically not only to VEGF, but also to MCP-1, it is possible that the response to multiple chemotactic cues will lead to a very different network structures compared to one determined by the distribution of VEGF and the response of ECs alone. One component of a model based on this scenario is chemotaxis in response to multiple signals, a process that is still poorly understood (Painter et al., 2000).

9. The role of angiogenic inhibitors A large number of factors can switch angiogenesis on or off, and a major issue in current angiogenesis research concerns the role and function of the various inhibitors that have been discovered. Mathematical modeling could play a significant role in pointing towards the right experimental designs that will further elucidate the role of the inhibitors. The only current mathematical models that explicitly incorporate the dynamics of angiostatin are those due to Levine et al. (2000); Levine et al. (2001a); Levine et al. (2001b) discussed earlier, and a much more detailed analysis than is presented therein is necessary. In view of the lack of experimental data, the models should aim at offering experimentally testable, qualitative insights into the function of the inhibitors. After significant intuition on the various possible roles of the inhibitors has been gained, modeling may help in understanding how the opposing signals that EC receive become integrated by the intracellular EC signal transduction network to produce migratory and proliferative responses. Furthermore, once the role of angiogenic inhibitors has been clarified, mathematical modeling can provide the basis for the quantitative design of inhibitor delivery strategies.

10. Blood flow through the capillary network The function of the newly developed vascular network in tumors is to supply the expanding mass of tumor cells with nutrients such as glucose and oxygen. However, the tumor vasculature also supplies a route for the delivery of anti-tumor drugs, and thus an important extension of the models discussed earlier is to explicitly incorporate fluid flow through the tumor vascular network. Ideally, one would like to model this process using realistic three dimensional tubular structures to represent the tumor capillaries. This model would then give a realistic description of the vasculature at the level of the capillary sprout, extending the idea of Stokes \& Lauffenburger (1991), and could then be used to study how vascular wall shear rates and changes in the viscosity of the blood, and concentration of drugs can effect the morphology and growth/or decay of the tumor network. Thus far, the usual approach for the modeling has been to follow the migration of a single cell (or density of cells) from the parent vessel to the tumor, without considering how the ECs might associate with each other to form a capillary lumen. A great deal of work is needed to extend the modeling from this simple description to the level of EC-lined tubular structures.

Recent work modeling the flow through vascular networks appears in McDougall et al. (2002), wherein the two- dimensional discrete approach of Anderson \& Chaplain (1998b) is used to generate the capillary networks. The resulting networks are given by the paths of the advancing capillary tip cells, and the authors then use flow modeling tools from the field of petroleum engineering to study the flow of the fluid through this interconnected network. They address issues such as how fluid viscosity and network geometry effects the rate of flow through the network and the amount of fluid reaching the tumor. This work highlights the importance of the structure and morphology of the network, in particular the connectedness of the network, for the delivery of chemotherapeutic drugs to the tumor.

Acknowledgments We are indebted to Marit Nilsen-Hamilton, Douglas Lauffenburger, Howard Levine, Brian Sleeman and Nicoleta Tarfulea for numerous comments, suggestions and corrections to earlier versions of this review. SDW would like to thank colleagues from the Loughborough University Mathematical Biology Group for helpful discussions. 


\section{Appendix: Glossary of Selected Terms and Abbreviations}

anastomose joining to form loops, interconnecting, as in capillary blood vessels.

angiogenesis the development of new blood vessels, especially in tissues where circulation has been impaired by trauma or disease, e.g cancer.

angiogenesis inhibitors agents and endogenous substances that antagonize or inhibit the development of new blood vessels, e.g. endostatin, angiostatin.

angiopoietins (Ang-1,-2) A family of molecules involved in angiogenesis. These molecules, together with their cell-surface tyrosine kinase receptors Tie-1 and Tie-2, play a key role in development of the vasculature and have been implicated in the control of vessel stabilisation and regression.

angiostatin a fragment of plasminogen with multiple anti-angiogenic activities in vitro and in vivo.

basement membrane (BM) thin layer of extracellular matrix lying between the epithelium and its underlying connective tissue.

capillaries the fi nest branches of blood vessels which connect small arteries and small veins.

capillary sprouting the formation of new capillary blood vessels by growth and migration of endothelial cells from existing blood vessels.

chemotaxis the directed movement of a microorganism or cell in response to a chemical stimulus.

collagen fi brous protein that is a major component of the extracellular matrix and connective tissues. These have a high tensile strength and exist in many forms; type I, the most common, is found in skin, tendon and bone; type II is found in cartilage; type IV is present in the basement membrane.

connective tissue supporting tissue that consists of cells embedded in a relatively large amount of extracellular matrix.

cytokine extracellular signaling protein that acts as a local mediator in cell-cell communication.

endostatin proteolytic fragment of collagen XVII that affects endothelial cell survival via induction of an imbalance between pro- and anti-apoptotic proteins.

endothelium single sheet of fhttened cells (endothelial cells, ECs) that forms the lining of all blood vessels. These regulate exchanges between the bloodstream and surrounding tissues and is usually surrounded by a basement membrane.

extracellular matrix (ECM) complex network of proteins (such as collagen) secreted by cells. Serves as a structural element in tissues.

fibrin protein that forms in the extracellular space by cleavage of blood plasma fi brinogen by the enzyme thrombin during blood clotting or vascular permeability/injury.

fibroblast growth factor (FGF) multi-functional growth factor present in several tissues in vivo and is synthezied by a number of cell types in vitro. Basic FGF (bFGF) can bind with high affi nity to heparin sulfate proteoglycans. Also known as heparin-binding factor.

fibronectin serves as an extracellular adhesion molecule by anchoring cells to collagen. Is secreted by fi broblasts, endothelial cells, macrophages, as well as certain epithelial cells.

growth factor an extracellular signaling molecule that stimulates a cell to grow or proliferate, e.g. vascular endothelial growth factor (VEGF) and fi broblast growth factor (FGF).

haptotaxis the movement of a cell up an adhesive gradient.

heparin sulfate proteoglycan an abundant component of most extracellular structures and can bind growth factors (such as TGF- $\beta$ s and FGFs), proteases and protease inhibitors. The plasma membrane (cell surface) proteoglycans can bind to ECM components.

hypoxia the reduction of oxygen levels

integrins member of the large family of transmembrane proteins involved in the adhesion of cells to the extracellular matrix.

in vitro the recreation of biological processes in an artifi cial laboratory environment.

in vivo biological processes that take place within a living organism or cell.

laminin an adhesive protein that is found mainly in the basement membrane.

macrophage a type of scavenger cell that engulfs cellular debris and damaged cells by phagocytosis. Some macrophages are free-moving, others are located in fi xed locations (e.g. Kupffer cells in the liver).

matrix metalloproteinase (MMPs) proteolytic enzymes that degrade extracellular matrix. MMPs share the common feature that they contain a zinc ion at their active site and are dependent on calcium for their catalytic activity. Based on their substrate specifi city MMPs can be divided into fi ve groups. They are inhibited by tissue inhibitors of matrix metalloproteinases (TIMPs).

mesenchymal cell mural precursor cell that is capable of replication as an undifferentiated cell or differentiating into connective tissue, blood vessel, or lymphatic cells.

monocyte type of white blood cell produced in the bone marrow. Monocytes remain in the blood stream for a short time and then migrate to tissues and mature into macrophages. 
monocyte chemotactic protein-1 (MCP-1) chemotactic and activating signal for monocytes, expressed mainly by tumor cells as well as ECs, fi broblasts, and macrophages.

mural cell smooth muscle cell or pericyte.

pericyte slender, contractile cells found in close contact with capillary walls. They are relatively undifferentiated and may become fi broblasts, macrophages, or smooth muscle cells.

plasmin a product of the lysis of plasminogen by plasminogen activators. It is the major proteolytic enzyme involved in blood clot retraction or the lysis of fi brin and quickly inactivated by antiplasmins.

plasminogen (pgn) inactive serine protease precursor that is abundant in the bloodstream and accumulates at sites of tissue remodeling such as wounds, tumors and sites of inflammation.

plasminogen activators (PA) serine proteinases that convert the zymogen plasminogen to plasmin. Urokinase-type (uPA) and tissue-type (tPA) are the principal activators of plasminogen.

plasminogen activator inhibitor (PAI) a member of the serpin family of proteins. It inhibits both the tissue-type and urokinase-type plasminogen activators.

platelet-derived growth factor (PDGF) a protein, produced by platelets and other cells, that strongly stimulates cell growth and division and is involved in mural cell recruitment in angiogenesis.

protease (proteinase, proteolytic enzyme) an enzyme that degrades proteins, such as collagen, laminin, and fi brin.

serine proteinase family of proteolytic enzymes that contain a serine residue at the active site of catalysis.

serpin serine proteinase inhibitors. The most important of which are plasminogen activator inhibitor-1 (PAI-1) and $\alpha_{2}$-plasmin inhibitor.

smooth muscle cell type of long, spindle-shaped muscle cell making up the muscular tissue found in the walls of arteries.

thrombospondin-1 (TSP-1) an extracellular matrix protein expressed by platelets and a variety of normal and transformed cells. Thrombospondin1 is believed to play a role in cell migration and proliferation during angiogenesis and wound repair.

tissue-type plasminogen activator a proteolytic enzyme in the serine protease family found in many tissues which converts plasminogen to plasmin. The primary role of tPA is to generate plasmin during blood clotting, while it is mainly uPA which generates plasmin for ECM degradation.

transforming growth factor beta (TGF- $\beta$ ) synthesized in a wide variety of tissues including platelets, placenta, and both normal and transformed cell lines. TGF- $\beta$ has a potential role in embryonic development, cellular differentiation, hormone secretion and immune function. Shown to act as a negative autocrine growth factor.

tumor angiogenic factor (TAF) a substance causing proliferation of new blood vessels. Found in hypoxic tissues, or tissues with high metabolic requirements, such as in certain cancers.

tumor-associated macrophage (TAM) macrophages that are attracted to solid tumor sites.

urokinase a proteolytic enzyme that converts plasminogen to plasmin. It is immunologically different from tissue plasminogen activator, and it is mostly uPA which has been studied in relation to angiogensis.

vascular endothelial growth factor (VEGF; also called vascular permeability factor, VPF) an endothelial-cell specifi c mitogen that has been shown to be a critical regulator of vascular development and growth.

vacuole membrane fluid fi lled space within a cell.

VE-cadherin cell junction in which the cytoplasmic face of the plasma membrane is attached to actin filaments. Acts as an adhesion junction linking adjacent endothelial cells.

vitronectin (VN) a blood plasma protein that mediates cell adhesion and interacts with proteins of the PA/Plasmin system.

zymogen an enzyme precursor molecule that may be catalytically transformed into an enzyme. Also called 'proenzyme'. 


\section{References}

Adam, John A., \& Bellomo, N. 1997. A Survey of Models for Tumor-Immune System Dynamics. Boston: Birkhäuser.

Ahmad, S. A., Liu, W., Jung, Y. D., Fan, F., Reinmuth, N., Bucana, C. D., \& Ellis, L. M. 2001. Differential expression of angiopoietin-1 and angiopoietin-2 in colon carcinoma. A possible mechanism for the initiation of angiogenesis. Cancer, 92(5), $1138-1143$.

Aiello, L. P., Pierce, E. A., Foley, E. D., Takagi, H., Chen, H., Riddle, L., Ferrara, N., King, G. L., \& Smith, L. E. 1995. Suppression of retinal neovascularization in vivo by inhibition of vascular endothelial growth factor (VEGF) using soluble VEGF-receptor chimeric proteins. Proc. Natl. Acad. Sci. USA, 92, 104570-10461.

Alberts, Bruce, Johnson, Alexander, Lewis, Julian, Raff, Martin, Roberts, Keith, \& Walter, Peter. 2002. Molecular Biology of The Cell. Fourth edn. New York and London: Garland.

Allt, G., \& Lawrenson, J. G. 2001. Pericytes: cell biology and pathology. Cells Tissues Organs, 169(1), 1-11.

Anderson, A. R. A., \& Chaplain, M. A. J. 1998a. A mathematical model for capillary network formation in the absence of endothelial cell proliferation. Appl. Math. Lett., 11, 109-114.

Anderson, A. R. A., Chaplain, M. A. J., Garcia-Reimbert, C., \& Vargas, C. A. 2000. A gradient driven mathematical model of antiangiogenesis. Mathl. Comput. Modeling, 32, 1141-1152.

Anderson, Alexander R. A., \& Chaplain, M. A. J. 1998b. Continuous and discrete mathematical models of tumor-induced angiogenesis. 60(5), 857-899.

Andreasen, P. A., Kjoller, L., Christensen, L., \& Duffy, M. J. 1997. The urokinase-type plasminogen activator system in cancer metastasis: a review. Int J Cancer, 72, 1-22.

Andres, J. L., Stanley, K., Cheifetz, S., \& Massague, J. 1989. Membrane-anchored and soluble forms of betaglycan, a polymorphic proteoglycan that binds to transforming growth factor-? J. Cell Biol, 109, 3137-3145.

Angiolillo, A. L., Sgadari, C., Taub, D. D., Liao, F., Farber, J. M., Maheshwar, S., Kleinman, H. K., Reaman, G. H., \& Tosato, G. 1995. Human interferon-inducible protein 10 is a potent inhibitor of angiogenesis in vivo. J. Exp. Med., 182, 155-162.

Asahara, T., Chen, D., Takahashi, T., Fujikawa, K., Kearney, M., Magner, M., Yancopoulos, G. D., \& Isner, J. M. 1998. Tie2 receptor ligands, angiopoietin-1 and angiopoietin-2, modulate VEGF-induced postnatal neovascularization. Circ Res, 83(3), 233-240.

Assoian., R. K., Komoriya, A., Meyers, C. A., Miller, D. M., \& Sporn, M. B. 1983. Transforming growth factor-beta in human platelets. J. Biol. Chem, 258, 7155-7160.

Ausprunk, D. H., \& Folkman, J. 1977. Migration and proliferation of endothelial cells in preformed and newly-formed blood vessels during tumor angiogenesis. Microvasc. Res, 14, 53-65.

Bach, T. L., Barsigian, C., Chalupowicz, D. G., Busler, D., Yaen, C. H., Grant, D. S., \& Martinez, J. 1998. VE-cadherin mediates endothelial cell capillary tube formation in fi brin and collagen gel. Exp. Cell Res, 238, 324-334.

Baish, J. W., Gazit, Y., Berk, D. A., Nozue, M., Baxter, L. T., \& Jain, R. K. 1996. Role of tumor vascular architecture in nutrient and drug delivery: An invasion percolation-based network model. Microvasc. Res, 51, 327-346.

Balding, D., \& McElwain, D. L. S. 1985. A mathematical model of tumour-induced capillary growth. J. theor. Biol, 114, 53-73.

Balsari, A., Maier, J. A. M., Colnaghi, M. I., \& Menard, S. 1999. Correlation between tumor vascularity, vascular endothelial growth factor production by tumor cells, serum vascular endothelial growth factor levels, and serum angiogenic activity in patients with breat carcinoma. Lab. Invest, 79, 897-902.

Barleon, B., Sozzani, S., Zhou, D., Weich, H. A., Mantovani, A., \& Marme, D. 1996. Migration of human monocytes in response to vascular endothelial growth factor (VEGF) is mediated via the VEGF receptor ft-1. Blood, 87(8), 3336-3343.

Baum, M., Chaplain, M. A. J., Anderson, A. R. A., Douek, M., \& Vaidya, J. S. 1999. Does breast cancer exist in a state of chaos? Eur. J. Cancer, 35, 886-891.

Bayless, K. J., Salazar, R., \& Davis, G. E. 2000. RGD-dependent vacuolation and lumen formation observed during endothelial cell morphogenesis in three -dimensional fi brin matrices involves the $\alpha_{w} \beta_{3}$ and $\alpha_{5} \beta_{1}$ integrins. Am. J. Pathol, 156, $1673-1683$.

Benjamin, L. E., Golijanin, D., Itin, A., Pode, D., \& Keshet, E. 1999. Selective ablation of immature blood vessels in established human tumors follows vascular endothelial growth factor withdrawal. J Clin Invest, 103(2), 159-165.

Bicknell, R. J., Lewis, C. E., Ferrara, N., \& Ferrara, L. (eds). 1997. Tumour Angiogenesis. Oxford: Oxford University Press.

Bingle, L., Brown, N. J., \& Lewis, C. E. 2002. The role of tumour-associated macrophages in tumour progression: implications for new anticancer therapies. J Pathol, 196(3), 254-265. Review.

Bloch, W., Huggel, K., Sasaki, T., Grose, R., Bugnon, P., Addicks, K., Timpl, R., \& Werner, S. 2000. The angiogenesis inhibitor endostatin impairs blood vessel maturation during wound healing. FASEB J., 14(15), 2373-2376.

Bohem, T., Folkman, J., Browder, T., \& O’Reilly, M. S. 1997. Antiangiogenic therapy of experimental cancer does not acquire dryg resistance. Nature, 390, 404-407. 
Bornstein, P. 1995. Diversity of function is inherent in matricellular protein: an appraisal of thrombospondin 1. J. Cell. Biol., 130, 503-506.

Bouck, N., Stellmach, V., \& Hsu, S. C. 1996. How tumors become angiogenic. Adv. Cancer Res, 69, 135-74.

Breier, G. 2000a. Angiogenesis in embryonic development-a review. Placenta, 21, S11-S15.

Breier, G. 2000b. Endothelial receptor tyrosine kinases involved in blood vessel development and tumor angiogenesis. Adv Exp Med Biol, 476, 57-66.

Brooks, P. C., Montgomery, A. M., Rosenfeld, M., Hu, T., Klier, G., \& Cheresh, D. A. 1994a. Integrin $\alpha_{v} \beta_{3}$ antagonists promote tumor regression by inducing apoptosis of angiogenic blood vessels. Cell, 79, 1157-1164.

Brooks, P. C., Clark, R. A., \& Cheresh, D. A. 1994b. Requirement of vascular integrin $\alpha \mathrm{v} \beta 3$ for angiogenesis. Science, 264, 569-571.

Brooks, P. C., Silletti, S., Schalscha, T. L. Von, Friedlander, M., \& Cheresh, D. A. 1998. Disruption of angiogenesis by PEX, a noncatalytic metalloproteinase fragment with integrin binding activity. Cell, 92, 391-400.

Brouty-Boye, D., \& Zetter, B. R. 1980. Inhibition of cell motility by interferon. Science, 208(4443), 516-518.

Brown, L. F., Detmar, M., Claffey, K., Nagy, J. A., Feng, D., Dvorak, A. M., \& Dvorak, H. F. 1997. VPF/VEGF: A multifunctional angiogenic cytokine. Chap. Regulation of Angiogenesis.

Byrne, H. M., \& Chaplain, M. A. J. 1995. Mathematical models for tumour angiogenesis: Numerical simulations and nonlinear wave solutions. Bull. Math. Biol, 57, 461-486.

Byrne, H. M., \& Chaplain, M. A. J. 1996. Explicit solutions of a simplifi ed model of capillary sprout growth during tumor angiogenesis. Appl. Math. Lett., 9(1), 69-74.

Byzova, T. V., Goldman, C. K., Pampori, N., Thomas, K. A., Bett, A., Shattil, S. J., \& Plow, E. F. 2000. A mechanism for modulation of cellular responses to VEGF: activation of the integrins. Mol. Cell, 6(4), 851-860.

Cao, Y. 2001. Endogenous angiogenesis inhibitors and their therapeutic implications. IJBCB, 33, 357-369.

Cao, Y., Chen, C., Weatherbee, J. A., Tsang, M., \& Folkman, J. 1995. Gro-beta, a-C-X-C- chemokine, is an angiogenesis inhibitor that suppresses the growth of lewis lung carcinoma in mice. J. Exp. Med., 182, 2069-2077.

Carlson, T. R., Feng, Y., Maisonpierre, P. C., Mrksich, M., \& Morla, A. O. 2001. Direct cell adhesion to the angiopoietins mediated by integrins. J. Biol. Chem., 276(28), 26516-26525.

Carmeliet, P., \& Jain, R. K. 2000. Angiogenesis in cancer and other diseases. Nature, 407, 249-257.

Chaplain, M. A. J. 2000. Mathematical modelling of angiogenesis. Journal of Neuro-Oncology, 50, 37-51.

Chaplain, M. A. J., \& Anderson, A. R. A. 1996. The mathematical modeling, simulation and prediction of tumor-induced angiogenesis. Invas. Metast, 16, 222-234.

Chaplain, M. A. J., \& Anderson, A. R. A. 1999. Modeling the growth and form of capillary networks. Chichester: Wiley. Chap. On Growth and Form: Spatio-Temporal Pattern Formation in Biology, pages 225-249.

Chaplain, M. A. J., \& Orme, M. E. 1998. Mathematical modeling of tumor-induced angiogenesis. Boston: Birkhauser. Chap. Vascular Morphogenesis: In vivo, in vitro, in mente, pages 205-240.

Chaplain, M. A. J., Giles, S. M., Sleeman, B. D., \& Jarvis, R. J. 1995. A mathematical analysis of a model for tumour angiogenesis. J. Math. Biol, 33, 744-770.

Chen, C., Parangi, S., Tolentino, M. J., \& Folkman, J. 1995. A strategy to discover circulating angiogenesis inhibitors generated by human tumors. Cancer Res., 55, 4230-4233.

Christofori, G. 1996. The Role of Fibroblast Growth Factors in Tumor Progression and Angiogenesis. R. bicknell, ce lewis and n. ferrara edn. Oxford University Press. Chap. Tumor Angiogenesis.

Claesson-Wells, L., Wells, M., Ito, N., Anand-Apte, B., Soker, S., Zetter, B., O’Reilly, S., \& Folkman, J. 1998. Angiostatin induces endothelial cell apoptosis and activation of focal adhesion kinase independently of the integrin-binding motif RGD. PNAS USA, 95, 5579-5583.

Clapp, C., Martial, J. A., Guzman, R. C., Rentierdelrue, F., \& Weiner, R. 1993. The 16-kilodalton N-terminal fragment of human prolactin is a potent inhibitor of angiogenesis. endocrinology, 133, 1292-1299.

Coleman, K. R., Braden, G. A., \& et al., M. C. Willingham. 1999. Vitaxin, a humanized monoclonal antibody to the vitronectin receptor ( $\alpha$ v $\beta 3$ ), reduces neointimal hyperplasia and total vessel area after balloon injury in hypercholesterolemic rabbits. Circ. Res., 84, $1268-1276$.

Colorado, P. C., Torre, A., Kamphaus, G., Maeshima, Y., Hopfer, H., Takahashi, K., Volk, R., Zamborsky, E. D., Sarkar, S. Herman P. K., Ericksen, E. M., Dhanabal, M., Simons, M., Post, M., Kufe, D. W., Weichselbaum, R. R., Sukhatme, V. P., \& Kalluri, R. 2000. Anti-angiogenic cues from vascular basement membrane collagen. Cancer Res., 60, 2520-2526.

Cory, A. H., Owen, T. C., Barltrop, J. A., \& Cory, J. G. 1991. Use of an aqueous soluble tetrazolium/formazan assay for cell growth assays in culture. Cancer Commun, 3, 207-212. 
Coussens, L. M., \& Werb, Z. 1996. Matrix metalloproteinases and the development of cancer. Chem. Biol., 3(11), 895-904.

Dallon, J. C., \& Othmer, H. G. 1997. A discrete cell model with adaptive signalling for aggregation of dictyostelium discoideum. Philos Trans $R$ Soc Lond B Biol Sci, 352(1351), 391-417.

D’Amato, R. J., Loughnan, M. S., \& et al., E. Flynn. 1994. Thalidomide is an inhibitor of angiogenesis. Proc. Natl. Acad. Sci. USA, 91, 4082-4085.

Dameron, K. M., Volper, O. V., Tainsky, M. A., \& Bouck, N. 1994. Control of angiogenesis in fi broblasts by p53 regulation of thrombospondin-1. Science, 265, 1582-1584.

Davis, G. E., \& Camarillo, C. W. 1996. An $\alpha_{2} \beta_{1}$ integrin-dependent pinocytic mechanism involving intracellular vacuole formation and coalescence regulates capillary lumen and tube formation in three-dimensional collagen matrix. Exp. Cell Res, 224, 39-51.

Detmar, M. 2000. The role of VEGF and thrombospondins in skin angiogenesis. J Dermatol Sci, 24, S78-S84.

Dhanapal, M., Ramchandran, R., Waterman, M. J., Lou, H., Knebelmann, B., Segal, M., \& Sukhatme, V. P. 1999. Endostatin induces endothelial cell apoptosis. J.Biol. Chem., 274, 11721-11726.

Doki, Y., Shiozaki, H., Tahara, H., Imoue, M., Iihara, H. Oka K., Kadowaki, T., Takeichi, M., \& Takesada, M. 1993. Correlation between E-cadherin expression and invasiveness in vitro in human esophageal cancer cell line. Cancer Res, 53, 3421-3426.

Dubois-Stringfellow, N., Jonczyk, J., \& Bautch, V. L. 1994. Perturbations in the fi brinolytic pathway aboliah cyst formation but not capillary-like organization of cultured murine endothelial cells. Blood, 83, 3206-3217.

Dvorak, H. F., Brown, L. F., Detmar, M., \& Dvorak, A. M. 1995. Vascular permeability factor/vascular endothelail growth factor, microvascular hyperpermeability, and angiogenesis. AM. J. Pathol, 146, 1029-1039.

Eliceiri, B. P., Paul, R., Schwartzberg, P. L., Hood, J. D., Leng, J., \& Cheresh, D. A. 1999. Selective requirement for src kinases during VEGF-induced angiogenesis and vascular permeability. Mol. Cell, 4(6), 915-924.

Enholm, B., Paavonen, K., Ristimaki, A., Kumar, V., Gunji, Y., Klefstrom, J., Kivinen, L., Laiho, M., Olofsson, B., Joukov, V., Eriksson, U., \& Alitalo, K. 1997. Comparison of VEGF, VEGF-B, VEGF-C and ang-1 mRNA regulation by serum, growth factors, oncoproteins and hypoxia. Oncogene, 14(20), 2475-2483.

Ergun, S., Kilic, N., Wurmbach, J. H., Ebrahimnejad, A., Fernando, M., Sevinc, S., Kilic, E., Chalajour, F., Fiedler, W., H., Lauke, Lamszus, K., Hammerer, P., Weil, J., Herbst, H., \& Folkman, J. 2001. Endostatin inhibits angiogenesis by stabilization of newly formed endothelial tubes. Angiogenesis, 4(3), 193-206.

Etoh, T., Shibuta, K., Barnard, G. F., Kitano, S., \& Mori, M. 2000. Angiogenin expression in human colorectal cancer: the role of focal macrophage infi ltration. Clin Cancer Res, 6(9), 3545-3551.

Ferrara, N. 1999. Role of vascular endothelial growth factor in the regulation of angiogenesis. Kidney Int, 56, $794-814$.

Ferrara, N. 2000. VEGF: an update on biological and therapeutic aspects. Curr Opin Biotechnol, 11, 617-624.

Ferrara, N., \& Davis-Smyth, T. 1997. The biology of vascular endothelial growth factor. Endocr. Rev, 18, 4-25.

Ferrara, N., \& Keyt, B. 1997. VEGF: Basic biology and clinical implications. Chap. Regulation of Angiogenesis.

Flaumenhaft, R., Moscatelli, D., Saksela, O., \& Rifkin, D. B. 1989. Role of extracellular matrix in the action of basic fi broblast growth factor: matrix as a source of growth factor for long-term stimulation of plasminogen activator production and DNA synthesis. J. Cell Physiol, 75-81.

Folkman. 1986. The vascularization of tumors. Cancer Biology: Readings from Scientific American, 115-124.

Folkman, J. 1971. Therapeutic implications. N. Engl. J. Med, 285, 1182-1186.

Folkman, J. 1976. The vascularization of tumors. Sci. Am., 234, 58-64.

Folkman, J. 1995a. Angiogenesis in cancer, vascular, rheumatoid and other disease. Nature Med, 1, $27-31$.

Folkman, J. 1995b. Clinical applications of research on angiogenesis. N. Engl. J. Med, 333, 1757-1763.

Folkman, J., \& Haudenschild, C. 1980. Angiogenesis in vitro. Nature, 288, 551-556.

Folkman, J., \& Klagsbrun, M. 1987. Angiogenic factors. Science, 235, 442.

Folkman, J., Mendelsohn, J., Howley, P. M., Israel, M. A., \& Liotta, L. A. 1995. Tumor angiogensis. in: The molecular basis of cancer. Eds. WB Saunders:Philadelphia, 206-232.

Fong, G. H., Rossant, J., Gertenstein, M., \& Breitman, M. L. 1995. Role of the ft-1 receptor tyrosine kinase in regulating assembly of vascular endothelium. Nature, 376, 66-70.

Fong, T. A., Shawver, L. K., \& et al., L. Sun. 1999. SU5416 is a potent and selective inhibitor of the vascular endothelial growth factor receptor (fk-1/KDR) that inhibits tyrosine kinase catalysis, tumor vascularization. Cancer Res., 59, 9-106.

Franz, C. M., Jones, G. E., \& Ridley, A. J. 2002. Cell migration in development and disease. Dev Cell, 2(2), $153-158$.

Frater-Schroeder, M., Mueller, G., Birchmeier, W., \& Boehlen, P. 1986. Transforming growth factor- $\beta$ inhibits endothelial cell proliferation. Biochem. Biophys. Res. Commun, 137:295. 
Frazier, W. A. 1987. Thrombospondin: A modular adhesive glycoprotein of platelets and nucleated cells. J. Cell. Biol., 105, 625-632.

Frolik, C. A., Dart, L. L., Meyers, C. A., Smith, D. M., \& Sporn, M. B. 1983. Purifi cation and initial characterization of a type beta transforming growth factor from human placenta. Proc. Natl. Acad. Sci, 80, 3676-3680.

Fukata, Y., Amano, M., \& Kaibuchi, K. 2001. Rho-rho-kinase pathway in smooth muscle contraction and cytoskeletal reorganization of non-muscle cells. Trends Pharmacol Sci, 22(1), 32-39.

Funamoto, S., Milan, K., Meili, R., \& Firtel, R. A. 2001. Role of phosphatidylinositol 3' kinase and a downstream pleckstrin homology domain-containing protein in controlling chemotaxis in dictyostelium. Journal of Cell Biology, 153(4), 795-810.

Furumatsu, T., Yamaguchi, N., Nishida, K., Kawai, A., Kunisada, T., Namba, M., \& and, H. Inoue. 2002. Ninomiya Y. endostatin inhibits adhesion of endothelial cells to collagen I via alpha(2)beta(1) integrin, a possible cause of prevention of chondrosarcoma growth. $J$ Biochem (Tokyo), 131(4), 619-626.

Gamble, J. R., Matthias, L. J., Meyer, G., Kaur, P., Russ, G., Faull, R., Berndt, M. C., \& Vadas, M. A. 1993. Regulation of in vitro capillary tube formation by anti-integrin antibodies. J. Cell Biol, 121, 931-943.

Gazit, Y., Berk, D. A., Leunig, M., \& andR. K. Jain, L. T. Baxter. 1995. Scale-invariant behavior and vascular network formation in normal and tumor tissue. Phys. Rev. Lett, 75, 2428-2431.

Gerber, H. P., Hillan, K. J., Ryan, A. M., Kowalski, J. K., Keller, G. A., Rangell, L., Wright, B. D., Radtke, F., Aguet, M., \& Ferrara, N. 1999. VEGF is required for growth and survival in neonatal mice. Development, 126, 1149-1159.

Gimborne, M. A., Cotran, R. S., Leapman, S. B., \& Folkman, J. 1974. Tumor growth and neovasularization: An experimental model using the rabbit cornea. J. Nat. Can. Inst., 52, 413-427.

Goede, V., Brogelli, L., Ziche, M., \& Augustin, H. G. 1999. Induction of inflammatory angiogenesis by monocyte chemoattractant protein1. Int J Cancer, 82(5), 765-770.

Good, D. J., Polverini, P. J., Rastinejad, F., Beau, M. M. Le, Lemons, R. S., Frazier, W. A., \& Bouck, N. P. 1990. A tumor suppressordependent inhibitor of angiogenesis is immunologically and functionally indistinguishable from a fragment of thrombospondin. Proc. Natl. Acad. Sci. USA, 87, 6624-6628.

Gospodarowicz, D., Ferrara, N., Schweigerer, L., \& Neufeld, G. 1987. Structual characterization and biological functions of fi broblast growth factor. Endocr. Rev, 8, 95-113.

Goto, F., Goto, K., Weindel, K., \& Folkman, J. 1993. Synergistic effects of vascular endothelial growth factor and basic fi broblast growth factor on the proliferation and cord formation of bovine capillary endothelial cells within collagen gels. Lab. Invest, 69, $508-517$.

Griffi oen, A. W., \& Molema, J. 2000. Angiogenesis: Potentials for pharmacologic intervention in the treatment of cancer, cardiovascular diseases, and chronic inflammation. Pharmacological Reviews, 52(2), 237-268. Review.

Gupta, S. K., Hassel, T., \& Singh, J. P. 1995. A potent inhibitor of endothelial cell proliferation is generated by proteolytic cleavage of the chemokine platelet factor 4. Proc. Natl. Acad. Sci. USA, 92, 7799-7803.

Han, Z. C., \& Liu, Y. 1999. Angiogenesis:state of the art. Int. J. Hematology, 70, 68-82.

Hanahan, D. 1997. Signaling vascular morphogenesis and maintenance. Science, 227, 48-50.

Hanahan, D., \& Folkman, J. 1996. Patterns and emerging mechanisms of the angiogenic switch during tumorigenesis. Cell, 86, 353-364.

Hanai, J., Dhanabal, M., Karumanchi, S. A., Albanese, C., Waterman, M., \& and, B. Chan. 2002. Ramchandran R, pestell R, sukhatme VP. endostatin causes G1 arrest of endothelial cells through inhibition of cyclin D1. J Biol Chem, 277(19), 16464-16469.

Higgs, H. N., \& Pollard, T. D. 2001. Regulation of actin fi lament network formation through ARP2/3 complex: activation by a diverse array of proteins. Annu Rev Biochem, 70, 649-676. Review.

Hildenbrand, R., Wolf, G., Bohme, B., Bleyl, U., \& Steinborn, A. 1999. Urokinase plasminogen activator receptor (CD87) expression of tumor-associated macrophages in ductal carcinoma in situ, breast cancer, and resident macrophages of normal breast tissue. $J$ Leukoc Biol, 66(1), 40-49.

Hirschi, K. K., \& D’Amore, P. A. 1996. Pericytes in the microvasculature. Cardiovasc Res, 32(4), 687-698.

Hirschi, K. K., Rohovsky, S. A., \& D'Amore, P. A. 1997. Cell-cell interactions in vessel assembly: a model for the fundamentals of vascular remodelling. Transpl Immunol, 3, 177-178.

Hirschi, K. K., Rohovsky, S. A., Beck, L. H., Smith, S. R., \& D’Amore, P. A. 1999. Endothelial cells modulate the proliferation of mural cell precursors via platelet-derived growth factor-BB and heterotypic cell contact. Circ Res, 84(3), 298-305.

Holmes, M. J., \& Sleeman, B. D. 2000. A mathematical model of tumour angiogenesis incorporating cellular traction and viscoelastic effects. Journal of Theoretical Biology, 202(2), 95-112.

Homandberg, G. A., Williams, J. E., Grant, D. B. S., \& Eisenstein, R. 1985. Heparin-binding fragments of fi bronectin are potent inhibitors of endothelial cell growth. Am. J. Pathol, 120,327-332.

Houck, K. A., Ferrara, N., Winer, J., Li, B., \& Leung, D. W. 1991. The vascular endothelial growth factor family: identifi cation of a fourth molecular species and characterization of alternative splicing of RNA. Mol. Endocrinol., 5, 1806-1814. 
Houck, K. A., Leung, D. W., Rowland, AM. A. M., Winer, J., \& Ferrara, N. 1992. Dual regulation of vascular endothelial growth factor bioavailability by genetic and proteolytic mechanisms. J. Biol. Chem., 267, 26031-26037.

Hynes, R. O. 1992. Integrins: versatility, modulation and signaling in cell adhesion. Cell, 69, 11-25.

Ingber, D., Fujita, T., Kishimoto, S., Sudo, K., Kanamaru, T., Brem, H., \& Folkman, J. 1990. Synthetic analogues of fumagillin that inhibit angiogenesis and suppress tumour growth. Nature, 348, 555-557.

Inoue, K., Perrotte, P., Wood, C. G., Slaton, J. W., Sweeney, P., \& Dinney, C. P. 2000. Gene therapy of human bladder cancer with adenovirus-mediated antisense basic fi broblast growth factor. Clin Cancer Res, 6, 4422-4431.

Jiang, M. C., Liao, C. F., \& Lee, P. H. 2001. Aspirin inhibits matrix metalloproteinase-2 activity, increases E-cadherin production, and inhibits in vitro invasion of tumor cells. Biochem Biophys Res Commun, 282, 671-677.

Jimenez, B., \& Volpert, O. V. 2001. Mechanistic insights on the inhibition of tumor angiogenesis. J. Mol. Med., 78, 663-672.

Johnson, D. E., \& Williams, L. T. 1993. Structural and functional diversity in the FGF receptor multigene family. Adv Cancer Res, 60(1-41), Review.

Kamphaus, G. D., Colorado, P. C., Panka, D. J., Hopfer, H., Ramchandran, R., Torre, A., Maeshima, Y., Mier, J. W., Sukhatme, V. P., \& Kalluri, R. 2000. Canstatin, a novel matrix-derived inhibitor of angiogenesis and tumor growth. J. Biol. Chem., 275, 1209-1215.

Karihaloo, A., Karumanchi, S. A., Barasch, J., Jha, V., Nickel, C. H., Yang, J., Grisaru, S., Busch, K. T., Nigam, S., Rosenblum, N. D., Sukhatme, V. P., \& Cantley, L. G. 2001. Endostatin regulates branching morphogenesis of renal epithelial cells and ureteric bud. Proc Natl Acad Sci USA, 98(22), 12509-12514.

Kiani, M., \& Hudetz, A. 1991. Computer simulation of growth of anastomosing microvascular networks. J. Theor. Biol., 150, 547-560.

Kim, K. J., Li, B., Winer, J., Armanini, M., Gillett, N., Phillips, H. S., HS, \& Ferrara, N. 1993. Inhibition of vascular endothelial growth factor-induced angiogenesis suppresses tumor growth in vivo. Nature, 362, 841-844.

Kim, Y. M., Jang, J. W., Lee, O. H., Yeon, J., Choi, E. Y., Kim, K. W., Lee, S. T., \& Kwon, Y. G. 2000. Endostatin inhibits endothelial and tumor cellular invasion by blocking the activation and catalytic activity of matrix metalloproteinase. Cancer Res, 60(19), 5410-5413.

Kiosses, W. B., Shattil, S. J., Pampori, N., \& Schwartz, M. A. 2001. Rac recruits high-affi nity integrin alphavbeta3 to lamellipodia in endothelial cell migration. Nat Cell Biol, 3(3), 316-320.

Koblizek, T. I., Weiss, C., Yancopoulos, G. D., Deutsch, U., \& Risau, W. 1998. Angiopoietin-1 induces sprouting angiogenesis in vitro. Curr Biol, 8(9), 529-532.

Koga, K., Todaka, T., Morioka, M., Hamada, J., Kai, Y., Yano, S., Okamura, A., Takakura, N., Suda, T., \& Ushio, Y. 2001. Expression of angiopoietin-2 in human glioma cells and its role for angiogenesis. Cancer Res, 61(16), 6248-6254.

Konerdig, M. A., Ackern, C. Van, Steinberg, F., \& Streffer, C. 1992. The development of the tumoue vascular system: 2-D and 3-D approaches to network formation in human xenografted tumours. M.e. maragoudakis et al edn. New York: Plenum Press. Chap. angiogenesis in health and disease.

Koolwijk, P., Hanemaaijer, R., \& Van Hinsbergh, V. W. M. 1998. Proteases and angiogenesis: Regulation of plasminogen activators and matrix metalloproteases by endothelial cells. NATO ASI series. Series A, Life sciences, 298, 241

Kremer, C., Breier, G., Risau, W., \& Plate, K. H. 1997. Up-regulation of fk-1/vascular endothelial growth factor receptor 2 by its ligand in a cerebral slice culture system. Cancer Res, 57, 3852-3859.

Kukk, E., Wartiovaara, U., Gunji, Y., Kaukonen, J., Buhring, H. J., \& and, I. Rappold. 1997. Matikainen MT, vihko P, partanen J, palotie A, alitalo K, alitalo R. analysis of tie receptor tyrosine kinase in haemopoietic progenitor and leukaemia cells. Br J Haematol, 98(1), 195-203.

Landini, G., \& Misson, G. 1993. Simulation of corneal neovascularization by inverted diffusion limited aggregation. Invest. Ophthalmol. Vis. Sci, 34, 1872-1875.

Lauffenburger, D. A., \& Horwitz, A. F. 1996. Cell migration: a physically integrated molecular process. Cell, 84(3), 359-369.

Lauren, J., Gunji, Y., \& Alitalo, K. 1998. Is angiopoietin-2 necessary for the initiation of tumor angiogenesis? Am J Pathol, 5(1333-1339), Review.

Lawrence, D. A., Pircher, R., Kryceve-Martinerie, C., \& Petzelbauer, P. 1984. Normal embryo fi broblasts release transforming growth factors in latent form. J Cell Physiol, 121, 184-188.

Lee, S. J., Jang, J. W., Kim, Y. M., Lee, H. I., Jeon, J. Y., Kwon, Y. G., \& Lee, S. T. 2002. Endostatin binds to the catalytic domain of matrix metalloproteinase-2. FEBS Lett, 519(1-3), 147-152.

Leek, R. D., Lewis, C. E., Whitehouse, R., Greenall, M., Clarke, J., \& Harris, A. L. 1996. Association of macrophage infi ltration with angiogenesis and prognosis in invasive breast carcinoma. Cancer Res, 56(20), 4625-4629.

Levine, H. A., Sleeman, B. D., \& Nilsen-Hamilton, M. 2000. A mathematical model for the roles of pericytes and macrophages in angiogenesis. I. the role of protease inhibitors in preventing angiogenesis. Math. Biosci., 168, 77-115. 
Levine, H. A., Pamuk, S., Sleeman, B. D., \& Nilsen-Hamilton, M. 2001a. Mathematical modeling of capillary formation and development in tumor angiogenesis: penetration into the stroma. Bulletin of Mathematical Biology, 63, 801-863.

Levine, H. A., Sleeman, B. D., \& Nilsen-Hamilton, M. 2001b. Mathematical modeling of the onset of capillary formation initiating angiogenesis. J. Math. Biol., 42, 195-238.

Levine, H. A., Tucker, A. L., \& Nilsen-Hamilton, M. 2002. A mathematical model for the role of cell signal transduction in the initiation and inhibition of angiogenesis. Growth Factors, 20(4), 155-175.

Lewis, J. S., Landers, R. J., Underwood, J. C., Harris, A. L., \& Lewis, C. E. 2000. Expression of vascular endothelial growth factor by macrophages is up-regulated in poorly vascularized areas of breast carcinomas. J Pathol, 192(2), 150-158.

Liekens, S., De Clercq, E., \& Neyts, J. 2001. Angiogenesis: regulators and clinical applications. Biochemical Pharmacology, 61, 253-270.

Liotta, L. A., \& Stetler-Stevenson, W. G. 1993. Principles of molecular cell biology of cancer: cancer metastasis. Philadelphia: Lippincott. Chap. Cancer: Principles and Practice of Oncology, pages 134-149.

Lipponen, P. K. 1996. Expression of cathepsin D in transitional cell bladder tumours. J Pathol, 178(1), 59-64.

Lobov, I. B., Brooks, P. C., \& Lang, R. A. 2002. Angiopoietin-2 displays VEGF-dependent modulation of capillary structure and endothelial cell survival in vivo. Proc Natl Acad Sci U S A, 99(17), 11205-11210.

Lowe, P. M., Lee, M. L., Jackson, C. J., To, S. S., Cooper, A. J., \& Schrieber, L. 1995. The endothelium in psoriasis. Br. J. Dermatol, 132, 497-505.

Lu, H., Mabilat, C., Yeh, P., Guitton, J. D., Li, H., Pouchelet, M., Shoevaert, D., Legrand, Y., Soria, J., \& Soria, C. 1996. Blockage of urokinase receptor reduces in vitro the mobility and the deformability of endothelial cells. FEBS Lett, 380, 21-24.

Lyons, R. M., Keski-Oja, J., \& Moses, H. L. 1988. Proteolytic activation of latent transforming growth factor-? from fi broblast-conditioned medium. J. Cell Physiol, 106, 1659-1665.

Maeshima, Y., Colorado, P. C., \& Kalluri, R. 2000. Two RGD-independent alpha vbeta 3 integrin binding sites on tumstatin regulate distinct anti-tumor properties. J. Biol. Chem., 275, 23745-23750.

Maione, T. E., Gray, G. S., Petro, J., Hunt, A. J., Donner, A. L., Bauer, S. I., Carson, H. F., \& Sharpe, R. J. 1990. Inhibition of angiogenesis by recombinant human platelet factor-4 and related peptides. Science, 247, 77-79.

Maisonpierre, P. C., Suri, C., Jones, P. F., Bartunkova, S., Wiegand, S. J., Radziejewski, C., Compton, D., McClain, J., Aldrich, T. H., Papadopoulos, N., Daly, T.J., Davis, S., Sato, T. N., \& Yancopoulos, G. D. 1997. Angiopoietin-2, a natural antagonist for tie2 that disrupts in vivo angiogenesis. Science, 277(5322), 55-60.

Mandriota, S. J., \& Pepper, M. S. 1998. Regulation of angiopoietin-2 mRNA levels in bovine microvascular endothelial cells by cytokines and hypoxia. Circ Res, 83(8), 852-859.

Manoussaki, D., Lubkin, S. R., Vernon, R. B., \& Murray, J. D. 1996. A mathematical model for the formation of vascular networks in vitro. Acta Biotheoretica, 44, 271-282.

Mantovani, A. 1994. Tumor-associated macrophages in neoplastic progression: a paradigm for the in vivo function of chemokines. Lab Invest, 1, 5-16.

Matsumura, T., Wolff, K., \& Petzelbauer, P. 1997. Endothelial cell tube formation depends on cadherin 5 and CD31 intercations with fi lamentous actin. J. Immunol, 158, 3408-3416.

McDougall, S. R., Anderson, A. R. A., Chaplain, M. A. J., \& Sherratt, J. A. 2002. Mathematical modelling of fbw through vascular networks: Implications for tumour-induced angiogenesis and chemotherapy strategies. Bull. Math. Biol., 64, 673-702.

Meyer, G. T., Matthias, L. J., Noack, L., Vadas, M. A., \& JR, J. R. Gamble. 1997. Lumen formation during angiogenesis in vitro involves phagocytic activity, formation and secretion of vacuoles, cell death, and capillary tube remodelling by different populations of endothelial cells. Anat. Record, 249(3), 327-340.

Mignatti, P., \& Rifkin, D. B. 1996. Plasminogen activators and matrix metalloproteinases in angiogenesis. Enzyme Protein, $117-137$.

Moldovan, N. I. 2002. Role of monocytes and macrophages in adult angiogenesis: A light at the tunnel's end. J. Hematother Stem Cell Res, 11(2), 179-194. Review.

Monier-Gavelle, F., \& Duband, J. L. 1995. Control of N-cadherin mediated intercellular adhesion in migrating neural crest cells in vitro. J. Cell Sci, 3839-3853.

Montesano, R., Pepper, M. S., Mohle-Steinlein, U., \& Wagner, L. Orci W. Risau E. F. 1990. Increased proteolytic activity is responsible for the aberrant morphogenetic behaviour of endothelial cells expressing middle T oncogene. Cell, 62, 435-445.

Moscatelli, D., \& Rifkin, D. B. 1988. Membrane and matrix localization of proteinases - a common theme in tumor-cell invasion and angiogenesis. Biochim Biophys Acta, 948(1), 67-85.

Moscatelli, D., Presta, M., Joseph-Silverstein, J., \& Rifkin, D. B. 1986. Both normal and tumor cells produce basic fi broblast growth factor. J. Cell Physiol, 129, 273-276.

Murray, J. D., \& Oster, G. F. 1984. Cell traction models for generation of pattern and form in morphogenesis. J. Math. Biol, 19, $265-279$. 
Murray, J. D., \& Swanson, K. R. 1999. On Growth and Form: Spatio-Temporal Pattern Formation in Biology. Chichester: Wiley. Chap. On the mechanochemical theory of biological pattern formation with applications to wound healing and angiogenesis, pages 251-285.

Murray, J. D., Oster, G. F., \& Harris, A. K. 1983. A mechanical model for mesenchymal morphogenesis. J Math Biol., 17(1), $125-129$.

Muthukkaruppan, V. R., Kubai, L., \& Auerbach, R. 1982. Tumor-induced neovascularization in the mouse eye. J. Natl. Cancer Inst, 69, 699-705.

Nakahara, H., Howard, L., Thompson, E. W., Seiki, H. Sato M., Yeh, Y., \& Chen, W. 1997. Transmembrane/cytoplasmic domain-mediated membrane type 1-matrix metalloproteinase docking to invadopodia is required for cell invasion. Proc. Natl. Acad. Sci. USA, 94(15), 7959-7964.

Nekka, F., Kyriakos, S., Kerrigan, C., \& Cartilier, L. 1996. A model of growing vascular structures. Bull. Math. Biol., 58, $409-424$.

Neufeld, G., Cohen, T., Gengrinovitch, S., \& Gluzman-Poltorak, Z. 1999. Vascular endothelial growth factor (VEGF) and its receptors. FASEB J., 13, 9-22.

Norby, K. 1997. Angiogenesis: new aspects relating to its initiation and control. APMIS, 105, 417-437.

Norrby, K. 2002. Mast cells and angiogenesis. APMIS, 110(5), 355-71.

Olsen, Luke, Sherratt, Jonathan A., Maini, Philip K., \& Arnold, F. 1997. A mathematical model for the capillary endothelial cellextracellular matrix interactions in wound-healing angiogenesis. IMA journal of mathematics applied in medicine and biology, 14, 261-281.

O'Reilly, M. S. 1997. Angiostatin: An endogenous inhibitor of angiogenesis and of tumor growth. Chap. Regulation of Angiogenesis.

O'Reilly, M. S., Holmgren, L., Shing, Y., Chen, C., Rosenthal, R. A., Moses, M., Lane, W. S., Cao, Y., Sage, E. H., \& Folkman, J. 1994. Angiostatin: a novel angiogenesis inhibitor that mediates the suppression of metastases by a lewis lung carcinoma. Cell, 79, 315-328.

O’Reilly, M. S., Boehm, T., Shing, Y., Fukai, N., Vasios, G., Lane, W. S., Flynn, E., Birkhead, J. R., \& Olsen, J. Folkman B. R. 1997. Endostatin: An endogenous inhibitor of angiogenesis and tumor growth. Cell, 88, 277-285.

O'Reilly, M. S., Pirie-Shepherd, S., Lane, W. S., \& Folkman, J. 1999. Antiangiogenic activity of the cleaved conformation of the serpin antithrombin. Science, 285, 1926-1928.

Orlidge, A., \& D'Amore, P. A. 1987. Inhibition of capillary endothelial cell growth by pericytes and smooth muscle cells. J Cell Biol, $\mathbf{1 0 5}(3), 1455-1462$.

Orme, M. E., \& Chaplain, M. A. J. 1996. A mathematical model of the fi rst steps of tumour-related angiogenesis: Capillary sprout formation and secondary branching. IMA journal of mathematics applied in medicine and biology, 13, 73-98.

Orme, M. E., \& Chaplain, M. A. J. 1997. Two-dimensional models of tumour angiogenesis and anti-angiogenesis strategies. IMA Journal of Mathematics Applied in Medicine and Biology, 14, 189-205.

Oster, G. F., Murray, J. D., \& Harris, A. K. 1983. Mechanical aspects of mesenchymal morphogenesis. J. Embryol. Exp. Morph., 78, 83-125.

Othmer, Hans G., \& Stevens, Angela. 1997. Aggregation, blowup, and collapse: The ABC's of taxis in reinforced random walks. SIAM Journal on Applied Mathematics, 57(4), 1044-1081.

Painter, K. J., Maini, P. K., \& Othmer, H. G. 2000. Development and applications of a model for cellular response to multiple chemotactic cues. Journal of Mathematical Biology, 41(4), 285-314.

Papetti, M., \& Herman, I. M. 2002. Mechanisms of normal and tumor-derived angiogenesis. Am J Physiol Cell Physiol., 282(5), C947C970.

Parent, C. A., \& Devreotes, P. N. 1999. A cell's sense of direction. Science, 284(5415), 765-770. Review.

Patterson, B. C., \& Sang, Q. A. 1997. Angiostatin-converting enzyme activities of human matrilysin (MMP-7) and gelatinase B/type IV collagenase (MMP-9). J Biol Chem, 272(46), 28823-28825.

Paweletz, N., \& Knierim, M. 1989. Tumor-related angiogenesis. Critical Reviews in Oncology/Hematology, 9, $197-242$.

Pennington, D. W., Lopez, A. R., Thomas, P. S., \& Ryan, U. S. 1991. Stimulation of rat endothelial cell transforming growth factor-? production by bleomycin. J. Clin. Invest, 83, 148-154.

Pepper, M. S., \& Montesano, R. 1990. Proteolytic balance and capillary morphogenesis. Cell Diff. Develop, 32, $319-328$.

Pepper, M. S., Ferrara, N., Orci, L., \& Montesano, T. 1992. Potent synergism between vascular endothelial growth factor and basic fi broblast growth factor in the induction of angiogenesis in vitro. Biochem. Biophys. Res. Commun, 189, 824-831.

Pepper, M. S., Montesano, R., Mandriota, S. J., Orci, L., \& Vassalli, J. 1996. Angiogenesis: a paradigm for balanced extracellular proteolysis during cell migration and morphogenesis. Enzyme Protein, 49, 138-162.

Peters, K. G., Coogan, A., Berry, D., Marks, J., Iglehart, J. D., Kontos, C. D., Rao, P., Sankar, S., \& Trogan, E. 1998. Expression of tie2/tek in breast tumour vasculature provides a new marker for evaluation of tumour angiogenesis. Br J Cancer, 77(1), 51-56.

Pettet, G. J., Chaplain, M. A. J., McElwain, D. L. S., M., H., \& Byrne. 1996. A model of wound-healing angiogenesis in soft tissue. Mathematical Biosciences, 136(1), 35-63. 
Pike, S. E., Yao, L., Jones, K. D., Cherney, B., Appella, E., Sakaguchi, K., Nakhasi, H., Teruya-Feldstein, J., Wirth, P., Gupta, G., \& Tosato, G. 1998. Vasostatin, a calreticulin fragment, inhibits angiogenesis and suppresses tumor growth. J. Exp. Med., 188, 2349-2356.

Pircher, R., Jullien, P., \& Lawrence, D. 1986. Beta-transforming growth factor is stored in human blood platelets as a latent high molecular weight complex. Biochem. Biophys. Res. Commun, 136, 30-37.

Poltorak, Z., Cohen, T., \& Neufeld, G. 2000. The VEGF splice variants: properties, receptors, and usage for the treatment of ischemic diseases. Herz, 25, 126-129.

Polverini, P. J. 1996a. Cellular adhesion molecules. newly identifi ed mediators of angiogenesis. Am. J. Pathol., 148, $1023-1029$.

Polverini, P. J. 1996b. How the extracellular matrix and macrophages contribute to angiogenesis-dependent diseases. Eur J Cancer, 14, $2430-2437$.

Presta, M., Maier, J. A. M., \& Ragnotti, G. 1989. The mitogenic signally pathway but not the plasminogen activator-induced pathway of basic fi broblast growth factor is mediated through protein kinase $\mathrm{C}$ in fetal bovine aortic endothelial cells. J. Cell. Biol, 109, $1877-1884$.

Ramchandran, R., Dhanabal, M., Volk, R., Waterman, W. J., Segal, M., Lu, H., Knebelmann, B., \& Sukhatme, V. P. 1999. Antiangiogenic activity of restin, NC10 domain of human collagen XV: comparison to endostatin. Biochem. Biophys. Res. Commun., 255, $735-739$.

Rastinejad, F., Polverini, P. J., \& Bouck, N. P. 1989. Regulation of the activity of a new inhibitor of angiogenesis by a cancer suppressor gene. Cell, 56, 345-355.

Rehn, M., Veikkola, T., Kukk-Valdre, E., Nakamura, H., Ilmonen, M., \& and, C. Lombardo. 2001. Pihlajaniemi T, alitalo K, vuori K. interaction of endostatin with integrins implicated in angiogenesis. Proc Natl Acad Sci U S A, 98(3), 1024-1029.

Rifkin, D. B., \& Moscatelli, D. 1989. Recent developments in the cell biology of basic fi broblast growth factor. J. Cell Biol, 109, 1-6.

Ristimaki, A., Narko, K., Enholm, B., Joukov, V., \& Alitalo, K. 1998. Proinflammatory cytokines regulate expression of the lymphatic endothelial mitogen vascular endothelial growth factor-C. J Biol Chem, 273(14), 8413-8418.

Roberts, A. B., Sporn, M. B., Assoian, R. K., Smith, J. M., Roche, N. S., Wakefi eld, L. M., \& Heine, U. I. 1986. Transforming growth factor type beta: rapid induction of fi brosis and angiogenesis in vivo and stimulation of collagen formation in vitro. Proc. Natl. Acad. Sci. USA, 83, 4167.

Ruoslahti, E. 1996. How cancer spreads. Scientific American, 275, 150-154.

Saelman, E. U. M., Keely, P. J., \& Santoro, S. A. 1995. Loss of MDCK cell $\alpha_{2} \beta_{1}$ integrin expression results in reduced cyst formation, failure of hepatocyte growth factor scatter factor-induced branching morphogenesis, and increased apoptosis. J. Cell Sci, 108, 35313540 .

Saksela, O., \& Rifkin, D. B. 1990. Release of basic fi broblast growth factor-heparan sulfate complexes from endothelial cells by plaminogen activator-mediated proteolytic activity. J. Cell. Biol, 110, 767-775.

Saksela, O., Moscatelli, D., Sommer, A., \& Rifkin, D. B. 1998. Endothelial cell-derived heparan sulfate binds basic fi broblast growth factor and protects it from proteolytic degradation. J. Cell Biol, 107, 743-751.

Salcedo, R., Ponce, M. L., Young, H. A., Wasserman, K., Ward, J. M., Kleinman, H. K., Oppenheim, J. J., \& Murphy, W. J. 2000. Human endothelial cells express CCR2 and respond to MCP-1: direct role of MCP-1 in angiogenesis and tumor progression. Blood, 96(1), 34-40.

Sato, A., Iwama, A., Takakura, N., Nishio, H., Yancopoulos, G. D., \& Suda, T. 1998. Characterization of TEK receptor tyrosine kinase and its ligands, angiopoietins, in human hematopoietic progenitor cells. Int Immunol, 10(8), 1217-1227.

Sato, Y., \& Rifkin, D. B. 1988. Autocrine activities of basic fi broblast growth factor: regulation of endothelial cell movement, plasminogen activator synthesis, and DNA synthesis. J. cell Biol, 107, 1199-1205.

Sato, Y., \& Rifkin, D. B. 1989. Inhibition of endothelial cell movement by pericytes and smooth muscle cells: activation of a latent transforming growth factor-beta 1-like molecule by plasmin during co-culture. J Cell Biol, 109(1), 309-315.

Sato, Y., Tsubio, R., Lyons, R., Moses, H., \& Rifkin, D. B. 1990. Characterization of the activation of latent TGF- $\beta$ by co-cultures of endothelial cells and pericytes or smooth muscle cells: a self-regulating system. J. Cell. Biol, 111, 757-763.

Savill, Nicholas J., \& Hogeweg, Paulien. 1997. Modelling morphogenesis: From single cells to crawling slugs. Journal of Theoretical Biology, 184, 229-235.

Sawano, A., Iwai, S., Sakurai, Y., Ito, M., Shitara, K., Nakahata, T., \& Shibuya, M. 2001. Flt-1, vascular endothelial growth factor receptor 1, is a novel cell surface marker for the lineage of monocyte-macrophages in humans. Blood, 97(3), 785-791.

Schlessinger, J., Lax, I., \& Lemmon, M. 1995. Regulation of growth factor activation by proteoglycans: what is the role of the low affi nity receptors? Cell, 3(357-60), Review.

Schwartz, M. A., \& Ingber, D. E. 1994. Integrating with integrins. Mol. Biol. Cell, 5, 389-393.

Seandel, M., Noack-Kunnmann, K., Zhu, D., Aimes, R. T., \& Quigley, J. P. 2001. Growth factor-induced angiogenesis in vivo requires specifi c cleavage of fi brillar type I collagen. Blood, 97, 2323-2332. 
Senger, D. R., Claffey, K. P., Benes, J. E., Perruzzi, C. A., Sergiou, A. P., \& Detmar, M. 1997. Angiogenesis promoted by vascular endothelial growth factor: regulation through alpha1beta1 and alpha2beta1 integrins. Proc. Natl. Acad. Sci. USA, 94, $13612-13617$.

Sephy, P. 2000. Angiogenesis inhibitors in oncology: The research continues. Cancer Practice, 8(3), 148-150.

Servant, G., Weiner, O. D., Herzmark, P., Balla, T., Sedat, J. W., \& Bourne, H. R. 2000. Polarization of chemoattractant receptor signaling during neutrophil chemotaxis. Science, 287(5455), 1037-1040.

Sheetz, M. P., Felsenfeld, D., Galbraith, C. G., \& Choquet, D. 1999. Cell migration as a fi ve-step cycle. Biochem. Soc. Symp., 65, $233-243$.

Shen, B. Q., Lee, D. Y., \& et al, H. P. Gerber. 1998. Homologous up-regulation of KDR/FLK-1 receptor expression by vascular endothelial growth factor in vitro. J. Biol. Chem, 273, 29979-29985.

Shichiri, M., \& Hirata, Y. 2001. Antiangiogenesis signals by endostatin. FASEB J, 15(6), 1044-1053.

Sholley, M. M., Ferguson, G. P., Seibel, H. R., Montour, J. L., \& Wilson, J. D. 1984. Mechanisms of neovascularization. vascular sprouting can occur without proliferation of endothelial cells. Lab. Invest, 51(6), 624-634.

Sleeman, B. D., Anderson, A. R. A., \& Chaplain, M. A. J. 1999. A mathematical analysis of a model for capillary network formation in the absence of endothelial cell proliferation. Applied Mathematics Letters, 12, 121-127.

Stack, M. S., Gately, S., Bafetti, L. M., Enghild, J. J., \& Soff, G. A. 1999. Angiostatin inhibits endothelial and melanoma cellular invasion by blocking matrix-enhanced plasminogen activation. J. Biochem., 340, 77-84.

Stetler-Stevenson, W. G. 1999. Matrix metalloproteinases in angiogenesis: a moving target for therapeutic intervention. J Clin Invest, 9, 1237-1241.

Stokes, C. L., \& Lauffenburger, D. A. 1991. Analysis of the roles of microvessel endothelial cell random motility and chemotaxis in angiogenesis. J. theor. Biol, 152, 377-403.

Stratmann, A., Risau, W., \& Plate, K. H. 1998. Cell type-specifi c expression of angiopoietin-1 and angiopoietin-2 suggests a role in glioblastoma angiogenesis. Am J Pathol, 153(5), 1459-1466.

Tamada, V., Fukiage, C., Boyle, D. L., Azuma, M., \& Shearer, T. R. 2000. Involvement of cysteine proteases in bFGF-induced angiogenesis in guinea pig and rat cornea. J Ocular Pharmacol. Therapeutics, 16, 271-283.

Tanaka, S., Mori, M., Sakamoto, Y., Makuuchi, M. N., Sugimachi, K., \& Wands, J. R. 1999. Biological signifi cance of angiopoietin-2 expression in human hepatocellular carcinoma. J. Clin. Invest., 103, 341-345.

Taylor, S., \& Folkman, J. 1982. Protamine is an inhibitor of angiogenesis. Nature, 287, 307-312.

Tennant, T. R., Rinker-Schaeffer, C. W., \& Stadler, W. M. 2000. Angiogenesis inhibitors. Current Oncology Reports, 2, 11-16.

Thomas, W. E. 1999. Brain macrophages: on the role of pericytes and perivascular cells. Brain Res Brain Res Rev, 31(1), $42-57$.

Thompson, W. D., Li, W. W., \& Maragoudakis, M. 2000. The clinical manipulation of angiogenesis: pathology, side-effects, surprises and opportunities with novel human therapies. J. Pathol., 190, 330-337.

Thurston, G., Suri, C., Smith, K., McClain, J., Sato, T. N., Yancopoulos, G. D., \& McDonald, D. M. 1999. Leakage-resistant blood vessels in mice transgenically overexpressing angiopoietin-1. Science, 286(5449), 2511-2514.

Tischer, E., Mitchell, R., Hartman, T., Silva, M., Gospodarowicz, D., Fiddes, J. C., \& Abraham, J. A. 1991. The human gene for vascular endothelial growth-factor multiple protein forms are encoded through alternative exon splicing. J. Biol. Chem., 266, 11947-11954.

Toi, M., Inada, K., Suzuki, H., \& Tominaga, T. 1995. Tumour angiogenesis in breast cancer: its importance as a prognostic indicator and the association with vascular endothelial growth factor expression. Breast Cancer Res. Treat, 36, 193-204.

Tong, S., \& Yuan, F. 2000. Numerical simulations of angiogenesis in the cornea. Microvasc. Res., 1-14.

Trinchieri, G. 1998. Interleukin-12: a cytokine at the interface of inflammation and immunity. Adv Immunol, 70, 83-243.

Turing, A. M. 1952. The chemical basis of morphogenesis. Philosophical transactions of the Royal Society of London Series B, Biological sciences, 237, 37-72.

Ueno, T., Toi, M., Saji, H., Muta, M., Bando, H., Kuroi, K., Koike, M., Inadera, H., \& Matsushima, K. 2000. Signifi cance of macrophage chemoattractant protein-1 in macrophage recruitment, angiogenesis, and survival in human breast cancer. Clin Cancer Res, 6, 32823289.

Varner, J. A., Brooks, P. C., \& Cheresh, D. A. 1995. The integrin $\alpha_{\nu} \beta_{3}$ : angiogenesis and apoptosis. Cell Adhes. Commun., 3, 367-374.

Veikkola, T., \& Alitalo, K. 1999. VEGF's, receptors and angiogenesis. Seminars in Cacncer Biology, 9, 211-220.

Vernon, R. B., \& Sage, H. E. 1999. A novel, quantitative model for study of endothelial cell migration and sprout formation within three-dimensional collagen matrices. Microvasc. Res, 57, 118-133.

Voest, E. E., Kenyon, B. M., O’Reilly, M. S., Truitt, G., D’Amato, R. J., \& Folkman, J. 1995. Inhibition of angiogenesis in vivo by interleukin 12. Natl. Cancer Inst., 87, 581-586.

Volpert, O. V., Stellmach, V., \& Bouck, N. 1995. The modulation of thrombospondin and other naturally occurring inhibitors of angiogenesis during tumor progression. Breast Cancer Res Treat, 36, 119-126. 
Weninger, W., Pammer, J., Baghestanian, M., Uthman, M. Mildner A., Ballaun, C., Bankl, H. C., \& Valent, P. 1996. Expression of vascular endothelial growth factor (VEGF) by human mast cells. J. Vasc. Res, 33:422.

Werb, Z. 1997. ECM and cell surface proteolysis: Regulating cellular ecology. Cell, 91(4), 439-442.

Westermark, B., Siegbahn, A., Heldin, C. H., \& Claesson-Welsh, L. 1990. B-type receptor for platelet-derived growth factor mediates a chemotactic response by means of ligand-induced activation of the receptor protein-tyrosine kinase. Proc Natl Acad Sci U S A, 87(1), $128-132$.

Willam, C., Koehne, P., Jurgensen, J. S., Grafe, M., Wagner, K. D., Bachmann, S., \& and, U. Frei. 2000 . Eckardt KU. tie2 receptor expression is stimulated by hypoxia and proinflammatory cytokines in human endothelial cells. Circ Res, 87(5), 370-377.

Wong, A. L., Haroon, Z. A., Werner, S., Dewhirst, M. W., Greenberg, C. S., \& Peters, K. G. 1997. Tie-2 expression and phosphorylation in angiogenic and quiescent adult tissues. Circ. Res., 81, 567-574.

Wong, M. P., Cheung, K. N., Yuen, S. T., Fu, K. H., Chan, A. S., Leung, S. Y., \& Chung, L. P. 1998. Monocyte chemoattractant protein-1 (MCP-1) expression in primary lymphoepithelioma-like carcinomas (LELCs) of the lung. J Pathol, 186(4), 372-377.

Wu, Pl, Yonekura, H., Li, H., Nozaki, I., Tomono, Y., Naito, I., Ninomyia, Y., \& Yamamoto, H. 2001. Hypoxia down-regulates endostatin production by human microvascular endothelial cells and pericytes. Biochemical and Biophysical Research Communications, 288, $1149-1154$.

Yamagishi, S., Yonekura, H., Yamamoto, Y., Fujimori, H., Sakurai, S., \& and, N. Tanaka. 1999. Yamamoto H. vascular endothelial growth factor acts as a pericyte mitogen under hypoxic conditions. Lab Invest, 79(4), 501-509.

Yamagushi, N., Anand-Apte, B., Lee, M., Sasaki, T., Fukai, N., Shapiro, R., Que, I., Lowik, C., Timpl, R., \& Olsen, B. R. 1999. Endostatin inhibits VEGF-induced endothelial cell migration and tumor growth independently of zinc binding. The EMBO Journal, 18(16), $4414-4423$.

Yancopoulos, G. D., Davis, S., Gale, N. W., Rudge, J. S., Weigand, S. J., \& Holash, J. 2000. Vascular-specifi c growth factors and bloodvessel formation. Nature, 407(6801), 242-248. Review.

Yang, S., Graham, J., Kahn, J. W., Schwartz, E. A., \& Gerritsen, M. E. 1999. Functional roles for PECAM-1 (CD31) and VE-cadherin (CD144) in tube assembly and lumen formation in three-dimensional collagen gels. Am. J. Pathology, 155, 887-895.

Yuan, F., Chen, Y., Dellian, M., Safabakhsh, N., Ferrara, N., \& Jain, R. K. 1996. Time-dependent vascular regression and permeability changes in established human tumor xenografts induced by an anti-vascular endothelial growth factor/vascular permeability factor antibody. Proc. Natl. Acad. Sci. USA, 93, 14765-14770.

Zawicki, D. F., Jain, R. K., Shmid-Schoenbein, \& Chiem, S. 1981. Dynamics of neovascularization in normal tissue. Microvasc. Res, 21, 27-47.

Zhang, Y., Deng, Y., Luther, T., Muller, M., Ziegler, R., Waldherr, R., \& and, D. M. Stern. 1994. Nawroth PP. tissue factor controls the balance of angiogenic and antiangiogenic properties of tumor cells in mice. J Clin Invest, 94(3), 1320-1327.

Zhu, C., Bao, G., \& Wang, N. 2000. Cell mechanics: mechanical response, cell adhesion, and molecular deformation. Annu Rev Biomed Eng., 2, 189-226.

Zimmerman, K. 1923. Der peinere bau der blutcapillaren. A. Anat. Entwicklungsgesh., 68, 29-109. 